NBER WORKING PAPER SERIES

\title{
OPTIMAL INTEREST-RATE RULES: \\ II. APPLICATIONS
}

Marc P. Giannoni

Michael Woodford

Working Paper 9420

http://www.nber.org/papers/w9420

\author{
NATIONAL BUREAU OF ECONOMIC RESEARCH \\ 1050 Massachusetts Avenue \\ Cambridge, MA 02138 \\ December 2002
}

An earlier version of this work was delivered by the second author as the Jacob Marschak Lecture at the 2001 Far Eastern Meeting of the Econometric Society, Kobe, Japan, July 21, 2001. We thank Ed Nelson, Julio Rotemberg and Lars Svensson for helpful discussions, and the National Science Foundation, through a grant to the NBER, for research support. The views expressed herein are those of the authors and not necessarily those of the National Bureau of Economic Research.

(C) 2002 by Marc P. Giannoni and Michael Woodford. All rights reserved. Short sections of text not to exceed two paragraphs, may be quoted without explicit permission provided that full credit including, (C) notice, is given to the source. 
Optimal Interest-Rate Rules: I.I. Applications

Marc P. Giannoni and Michael Woodford

NBER Working Paper No. 9420

December 2002

JEL No. E52, E61

\section{$\underline{\text { ABSTRACT }}$}

In this paper we calculate robustly optimal monetary policy rules for several variants of a simple optimizing model of the monetary transmission mechanism with sticky prices and/or wages. We discuss representations of optimal policy both in terms of interest-rate feedback rules that generalize the well-known "Taylor rule," and in terms of commitment to a target criterion of the kind discussed in familiar proposals for "flexible inflation targeting."

Optimal rules, however, require that policy be history-dependent in ways not contemplated by many well-known proposals. We furthermore find that a robustly optimal policy rule is almost inevitably an implicit rule, that requires the central bank to use a structural model to project the economy's evolution under the contemplated policy action. Finally, our numerical examples suggest that optimal rules do not place nearly as much weight on projections of inflation or output many quarters in the future as occurs under the current practice of inflation-forecast targeting central banks.

\author{
Marc P. Giannoni \\ Graduate School of Business \\ Columbia University \\ New York, NY 10027 \\ mg2190@columbia.edu
}

Michael Woodford

Department of Economics

Princeton University

Princeton, NJ 08544

and NBER

woodford@princeton.edu 
In a companion paper (Giannoni and Woodford, 2002), we have expounded a general approach to the design of an optimal criterion on the basis of which a central bank should determine its operating target for the level of overnight interest rates. Our discussion there was framed in the context of a fairly general linear-quadratic policy problem. Here we consider the implications of our approach in the context of a particular (admittedly stylized) model of the monetary transmission mechanism, or rather a group of related variant models. ${ }^{1}$ This allows us to address a number of questions raised by recent characterizations of actual central-bank policies in terms of "Taylor rules" or "flexible inflation targets".

One basic question is whether it makes sense for a central bank's policy commitment to be formulated in terms of a relationship between the bank's interest-rate instrument, some measure of inflation, and some measure of an "output gap" that the bank seeks to ensure will hold - as is true both of the interest-rate rule recommended by Taylor (1993) and common formulations of "flexible inflation targeting" rules. Can a desirable policy rule be expressed without reference to a monetary aggregate? Can the rule be optimal despite a lack of any explicit dependence of policy upon the nature of the exogenous disturbances affecting the economy? If a desirable rule can be expressed in terms of a relation between these variables, which inflation measure, and which conception of the output gap should it involve? And how do the optimal coefficients of the respective variables depend on quantitative features of one's model of the monetary transmission mechanism?

A particular concern here will be with the optimal dynamic specification of a monetary policy rule. Taylor's well-known proposal prescribes a purely contemporaneous relation between the federal funds rate target, an inflation measure, and an output-gap measure; but estimated central-bank reaction functions (e.g., Judd and Rudebusch, 1998) always involve additional partial-adjustment dynamics for the funds rate, and sometimes other sorts of lagged responses as well. To what extent are such lags in the rule used to set interest

\footnotetext{
${ }^{1}$ While the general approach to the construction of robustly optimal policy rules used here is the same as that discussed in the companion paper, the derivations presented here are self-contained and do not rely upon any of the results for the general linear-quadratic problem presented in the earlier paper. It is our hope that a self-contained exposition of the relevant calculations for these simple models will serve to increase insight into the method, in addition to delivering results of interest with regard to these particular models.
} 
rate desirable? Some empirical studies (e.g., Clarida et al., 2000) imply that central banks respond to forecasts of the future levels of inflation and/or output rather than to current values. Is this preferable, and if so, how far in the future should these forecasts look?

We address these questions here along the lines proposed in Giannoni and Woodford (2002). Rather than optimizing over some parametric family of policy rules, we consider the design of a rule in order to bring about the optimal equilibrium pattern of responses to disturbances - more precisely, to determine an equilibrium that is optimal from the "timeless perspective" explained in our previous paper. In addition to requiring the rule to be consistent with this optimal equilibrium, we ask that the rule imply that rational expectations equilibrium should be determinate, so that commitment to the rule can be relied upon to bring about the desired equilibrium rather than some other, less desirable one. We also construct rules that can expressed purely in terms of the "target variables" that the central bank seeks to stabilize, and that are optimal regardless of the nature of the (additive) exogenous disturbances to which the economy is subject, and regardless of the statistical properties of those disturbances. The requirement that our rule simultaneously satisfy each of these desiderata allows us to narrow the class of optimal rules to a fairly small set; among these, we give primary attention to those rules that are simplest in form. Even so, we are typically left with more than one possible representation of optimal policy. In particular, in most of the cases considered here, optimal policy can be represented either by a generalized Taylor rule or by a history-dependent inflation target, and we discuss both of these formulations.

\section{Optimal Rules for a Simple Forward-Looking Model}

We first illustrate our method of constructing robustly optimal policy rules in the context of the basic optimizing model of the monetary transmission mechanism expounded in Woodford (2002, chap. 4), and used as the basis for the discussion of the optimal responses to real disturbances in Woodford (1999a) and Giannoni (2001). The model may be reduced to two 
structural equations

$$
\begin{gathered}
x_{t}=\mathrm{E}_{t} x_{t+1}-\sigma\left(i_{t}-\mathrm{E}_{t} \pi_{t+1}-r_{t}^{n}\right) \\
\pi_{t}=\kappa x_{t}+\beta \mathrm{E}_{t} \pi_{t+1}+u_{t}
\end{gathered}
$$

for the determination of the inflation rate $\pi_{t}$ and the output gap $x_{t}$, given the central bank's control of its short-term nominal interest-rate instrument $i_{t}$, and the evolution of the composite exogenous disturbances $r_{t}^{n}$ and $u_{t}$. Here the output gap is defined relative to an exogenously varying natural rate of output, chosen to correspond to the gap that belongs among the target variables in the central bank's loss function. The "cost-push shock" $u_{t}$ then represents exogenous variation in the gap between the flexible-price equilibrium level of output and this natural rate, due for example to time-varying distortions that alter the degree of inefficiency of the flexible-price equilibrium. ${ }^{2}$ The microfoundations for this model imply that $\sigma, \kappa>0$, and that $0<\beta<1$. The unconditional expectation of the natural rate of interest process is given by $E\left(r^{n}\right)=\bar{r} \equiv-\log \beta>0$, while the cost-push disturbance is normalized to have an unconditional expectation $E(u)=0$. Otherwise, our theoretical assumptions place no a priori restrictions upon the statistical properties of the disturbance processes, and we shall be interested in policy rules that are optimal in the case of a general specification of the additive disturbance processes of the form discussed in Giannoni and Woodford (2002, sec. 4).

The assumed objective of monetary policy is to minimize the expected value of a loss criterion of the form

$$
W=\mathrm{E}_{0}\left\{\sum_{t=0}^{\infty} \beta^{t} L_{t}\right\},
$$

where the discount factor $\beta$ is the same as in (1.2), and the loss each period is given by

$$
L_{t}=\pi_{t}^{2}+\lambda_{x}\left(x_{t}-x^{*}\right)^{2}+\lambda_{i}\left(i_{t}-i^{*}\right)^{2}
$$

for certain optimal levels $x^{*}, i^{*} \geq 0$ of the output gap and the nominal interest rate, and certain weights $\lambda_{x}, \lambda_{i}>0$. A welfare-theoretic justification is given for this form of loss

\footnotetext{
${ }^{2}$ See Woodford (2002, chap. 6) for discussion of the welfare-relevant output gap and of the nature of "cost-push shocks".
} 
function in Woodford (2002, chap. 6), where the parameters are related to those of the model of the structural model. However, our conclusions below are presented in terms of the parameters of the loss function (1.4), and are applicable in the case of any loss function of this general form, whether the weights and target values are the ones that can be justified on welfare-theoretic grounds or not. In the numerical results presented below, the model parameters are calibrated as in Table 1 of Woodford (1999a). (For convenience, the parameters are reported in Table 1 below.)

\subsection{The Optimal Taylor Rule}

Before turning to the question of fully optimal policy in this model, it may be of interest to briefly consider the optimal choice of a rule within a restricted class that has been widely discussed, which is to say, the class of "simple Taylor rules", 3

$$
i_{t}=\bar{\imath}+\phi_{\pi}\left(\pi_{t}-\bar{\pi}\right)+\phi_{x}\left(x_{t}-\bar{x}\right) / 4
$$

involving only contemporaneous feedback from the inflation rate and the output gap, and no direct responses to real disturbances. ${ }^{4}$ A rule of this form reflects the intuitive notion that it may be desirable to adjust the bank's instrument in response to deviations of its target variables (other than the instrument itself) from certain desired levels. The conditions for such a rule to imply a determinate equilibrium in this model have already been treated in Woodford (2002, chap. 4).

A rule of the form (1.5) represents an example of a purely forward-looking rule, so if it implies a determinate equilibrium, that equilibrium is one in which all three target variables

\footnotetext{
${ }^{3}$ In the rule proposed by Taylor (1993), the inflation variable is actually the most recent four-quarter change in the GDP deflator, whereas we here consider rules that respond to the change from the previous to the current quarter only. However, Taylor's intention seems to have been to assume feedback from contemporaneous measures of the Fed's (implicit) target variables. We here assume that the central bank seeks to stabilize the one-period inflation rate $\pi_{t}$ rather than some average of inflation over a longer time span, because this is the objective that we have been able to justify on welfare-theoretic grounds, in Woodford (2002, chap. 6). Our analysis is also simplest in this case, though similar methods could be used to analyze optimal policy in the case of an alternative inflation-stabilization objective, that might reflect the true goal of a particular central bank.

${ }^{4}$ The coefficient on the output gap is denoted $\phi_{x} / 4$ rather than $\phi_{x}$, so that $\phi_{x}$ corresponds to Taylor's output-gap coefficient, writing the rule in terms of annualized data. Here we assume that "periods" of our model correspond to quarters.
} 
will be functions solely of the current and expected future values of the real disturbances. Hence the best pattern of responses to disturbances that could possibly be implemented by a rule in this family is the one that we have called the optimal non-inertial plan in Woodford (1999a). In general, even the optimal non-inertial plan can only be implemented by a rule more complex than (1.5). One case in which a rule of this form suffices, however - at least for an open set of possible parameter values - is that in which both the $r_{t}^{n}$ and $u_{t}$ disturbances are Markovian (i.e., first-order autoregressive processes), as assumed in the numerical examples presented in Woodford (1999a) and Giannoni (2001). In such a case, the rule that implements the optimal non-inertial plan is clearly the optimal member of the family, and this makes calculation of the optimal Taylor rule quite straightforward.

Thus we assume once again disturbances of the form

$$
\begin{aligned}
& \hat{r}_{t}^{n}=\rho_{r} \hat{r}_{t-1}^{n}+\epsilon_{r t}, \\
& u_{t}=\rho_{u} u_{t-1}+\epsilon_{u t},
\end{aligned}
$$

where $\hat{r}_{t}^{n} \equiv r_{t}^{n}-\bar{r}, \epsilon_{r t}$ and $\epsilon_{u t}$ are i.i.d. mean-zero exogenous shocks, and $0 \leq \rho_{r}, \rho_{u}<1$. In this case, the constraints upon the feasible evolution of the target variables $\left\{\pi_{t}, x_{t}, i_{t}\right\}$ from date $t$ onward depend only upon the vector of current disturbances $e_{t} \equiv\left[\begin{array}{ll}\hat{r}_{t}^{n} & u_{t}\end{array}\right]^{\prime}$, and the optimal non-inertial plan is given by linear functions of the form

$$
z_{t}=\bar{z}+F e_{t}, \quad i_{t}=\bar{\imath}+f_{i} e_{t},
$$

where $z_{t} \equiv\left[\begin{array}{ll}\pi_{t} & x_{t}\end{array}\right]^{\prime}$ is the vector of endogenous variables other than the policy instrument. The long-run average values $\bar{z}, \bar{l}$ and response coefficients $F, f_{i}$ are given in the Appendix (section A.1).

In the (generic) case that the matrix $F$ is invertible, an instrument rule consistent with this pattern of responses to shocks is given by

$$
i_{t}=\bar{\imath}+f_{i} F^{-1}\left(z_{t}-\bar{z}\right)
$$

which takes the form of a simple Taylor rule (1.5). Note that while we have here written the rule in terms of deviations from implicit targets for each of the variables that correspond to 
the optimal long-run average values of these variables, the only thing that matters for the constrained optimality of (1.8) is the value of the total intercept term

$$
\bar{\imath}-f_{i} F^{-1} \bar{z}
$$

Of course, the above derivation guarantees only that the suggested rule is consistent with the equilibrium responses to shocks that constitute the optimal non-inertial plan. In order to implement the plan, we also need for the rule to imply a determinate equilibrium. The conditions under which this will be true have been discussed in Woodford (2002, chap. 4). The following result states conditions under which the coefficients of the rule just proposed satisfy this additional requirement .

Proposition 4. Suppose the disturbances are of the form (1.6) - (1.7), with autocorrelation coefficients satisfying the bounds

$$
\begin{aligned}
0 & <\left(1-\rho_{r}\right)\left(1-\beta \rho_{r}\right)-\rho_{r} \kappa \sigma \\
& \leq\left(1-\rho_{u}\right)\left(1-\beta \rho_{u}\right)-\rho_{u} \kappa \sigma<\frac{\kappa \sigma}{\lambda_{i}} .
\end{aligned}
$$

Then (1.8) defines a Taylor rule of the form (1.5) with coefficients $\phi_{\pi}>1, \phi_{x}>0$. Furthermore, commitment to this rule implies a determinate rational expectations equilibrium, which implements the optimal non-inertial plan.

The proof is given in the Appendix. Note that the inequalities assumed in the proposition may equivalently be written

$$
\underline{\rho}<\rho_{u} \leq \rho_{r}<\bar{\rho}
$$

where $\underline{\rho}<\bar{\rho}$ and the bounds are functions of the model parameters $\beta, \kappa, \sigma$ and $\lambda_{i}$. Thus there is an open set of values of $\rho_{r}$ and $\rho_{u}$ for which the conditions are satisfied, and these are not obviously unreasonable; for example, the calibrated values reported in Table 1 below satisfy these conditions. 
Under the conditions assumed in this last proposition, we thus obtain theoretical justification for crucial aspects of Taylor's recommendation. In particular, we provide support for his recommendation that the operating target for the federal funds rate should respond positively to fluctuations in both the current inflation rate and the current output gap. The rule proposed here also satisfies the "Taylor Principle", according to which an increase in inflation above the target rate results in an even greater increase in the nominal interest rate.

The need for non-zero response coefficients for both target variables follows from a desire to implement the optimal non-inertial responses to two distinct types of real disturbances — disturbances to the natural rate $r_{t}^{n}$ and "cost-push shocks" $u_{t}$ - or more precisely, to respond optimally to any of a range of real disturbances, which shift the model's structural equations in these two different ways to differing extents. If instead we assume that there are no cost-push shocks - not that there are no "supply" disturbances, but that all real disturbances shift the natural rate of output and the efficient rate of output to the same extent - then the requirement that our rule implement the optimal non-inertial response to disturbances to the natural rate of interest imposes only a single linear restriction upon the coefficients $\phi_{\pi}$ and $\phi_{x},{ }^{5}$ and it is possible to find a rule that implements the optimal non-inertial plan with $\phi_{x}=0$. On the other hand, adding the requirement that the rule also implement the optimal non-inertial response to cost-push shocks, should they ever occur, has no cost in terms of a less desirable response to disturbances to the natural rate of interest, ${ }^{6}$ and thus robustness concerns make it advisable that policy respond to variations in the output gap as well. Interestingly the optimal degree of response to variations in the output gap is independent of the assumed importance of cost-push shocks (i.e., the assumed variance of the $u_{t}$ disturbance); all that matters for the recommendation (1.8) is the assumed degree

\footnotetext{
${ }^{5}$ This case is analyzed in Woodford (1999a).

${ }^{6} \mathrm{We}$ assume here that there is no difficulty in measuring and hence responding to either of the target variables, inflation and the output gap. In practice, measurement of the output gap is likely to be more problematic, and for this reason implementation of the optimal non-inertial responses to variations in $r_{t}^{n}$ through a rule that involves a large coefficient $\phi_{x}$ may result in some deterioration in the ability of policy to successfully respond to those disturbances. The problem of the optimal conduct of policy when measurement problems are taken into account is considered below in section 3.3.
} 
of serial correlation of such disturbances when they occur.

Our analysis also provides at least partial support for Taylor's recommendation of what we have called a direct policy rule: one that specifies adjustment of the instrument purely in terms of feedback from the observed (or projected) behavior of the target variables. Of course we have not shown that the rule (1.8) cannot be improved upon; but we have shown that it is optimal within the class of purely forward-looking rules. This means that if we consider only possible dependence of the central bank's instrument upon various state variables that are relevant to the determination of current of future values of the target variables, there is no possible gain from introducing dependence upon variables other than those already allowed for in (1.5).

In particular, our analysis justifies Taylor's neglect of any response to projections of future inflation or output gaps, as opposed to projections for the current quarter. If we were to introduce additional terms representing feedback from $\mathrm{E}_{t} \pi_{t+j}$ or $\mathrm{E}_{t} x_{t+k}$ for some horizons $j, k>0$, the optimal rule within that broader family would achieve no better an outcome. For such a rule would continue to be purely forward-looking, and so could at best implement the optimal non-inertial plan, and this is already achieved by the optimal Taylor rule, under the assumptions of Proposition 4.

Nor is it even entirely correct to say that a forecast-based rule would be an equally useful way of achieving the same outcome. Under the assumption that the central bank has access to perfectly accurate forecasts (so that a forecast-based rule can, in principle, involve exactly the same equilibrium adjustment of interest rates as under a Taylor rule), then the optimal response coefficients become larger the longer the horizon of the forecasts that are used to implement policy. For example, suppose we consider forward-looking Taylor rules of the form

$$
i_{t}=\bar{\imath}+\phi_{\pi}\left(\mathrm{E}_{t} \pi_{t+k}-\bar{\pi}\right)+\phi_{x}\left(\mathrm{E}_{t} x_{t+k}-\bar{x}\right) / 4
$$

for a given forecast horizon $k>0$, and for simplicity assume that $\rho_{r}=\rho_{u}=\rho$, for some $0<\rho<1 .^{7}$ Then the unique rule within this family that is consistent with the optimal

\footnotetext{
${ }^{7}$ Note that in the case that either $\rho_{r}$ or $\rho_{u}$ is equal to zero, it will be impossible for a purely forecast-based
} 
non-inertial plan is given by

$$
i_{t}=\bar{\imath}+\rho^{-k} f_{i} F^{-1}\left(\mathrm{E}_{t} z_{t+k}-\bar{z}\right)
$$

both response coefficients must be multiplied by the factor $\rho^{-k}>1$, which may be quite large in the case of a horizon several quarters in the future. ${ }^{8}$ But such an alternative rule has the unpalatable feature that it involves a commitment to extremely strong responses to something that, in practice, is likely to be estimated with considerable error.

Furthermore, even when highly accurate conditional forecasts are available, a commitment to strong response to them by the central bank makes it likely that equilibrium will be indeterminate. ${ }^{9}$ For the class of forward-looking rules just considered, we can establish the following.

Proposition 5. For all forecast horizons $k$ longer than some critical value, the rule of the form (1.9) that is consistent with the optimal non-inertial plan implies indeterminacy of rational-expectations equilibrium.

The proof is in the Appendix. ${ }^{10}$ Thus if the forecast horizon $k$ is sufficiently long, it is not possible to implement the optimal non-inertial plan using a rule of the form (1.9). ${ }^{11}$ It follows that, at least when the parameters satisfy the conditions of Proposition 4, the best rule in this family is not as desirable as the best simple Taylor rule.

rule such as (1.9) to implement the optimal non-inertial plan, because under that pattern of responses to disturbances, the forecasts will not reveal information about the current value of the transitory disturbance.

${ }^{8}$ For example, if we assume a serial correlation coefficient of $\rho=.35$, as in the baseline calibration in Woodford (1999a), and a forecast horizon $k$ of 8 quarters, a fairly typical horizon for inflation-targeting central banks, this factor is greater than 4000 .

${ }^{9}$ Recall the discussion of this defect of forward-looking rules in Woodford (2002, chap. 4, sec. 2.2). The possibility that too strong a response to forecasts can lead to indeterminacy was first shown by Bernanke and Woodford (1997), while the possibility that too long a forecast horizon can lead to indeterminacy is illustrated by Levin et al.(2001).

${ }^{10}$ In independent recent work, Batini and Pearlman (2002) establish a related result for a general family of interest-rate feedback rules in which the current nominal interest rate operating target is a linear function of an inflation forecast and a lagged nominal interest rate. Our result here, however, is not strictly implied by the one that they state.

${ }^{11}$ For example, in the case of the calibrated parameter values given in Table 1 below, the rule (1.10) implies indeterminacy for all $k \geq 1$. 
In the case of the calibrated parameter values proposed in Table 1 below, including the values $\rho_{r}=\rho_{u}=.35$ for the serial correlation of the disturbance processes, the optimal Taylor rule is given by

$$
i_{t}^{a n n}=.03+1.72 \pi_{t}^{a n n}+.57 x_{t},
$$

where we now (for comparability with Taylor's prescription) report the rule in terms of an annualized interest rate and inflation rate $\left(i_{t}^{a n n}=4 i_{t}, \pi_{t}^{a n n}=4 \pi_{t}\right) .{ }^{12}$ These parameter values are quite similar to those recommended by Taylor. Particularly worthy of note is the substantial response coefficient $\phi_{x}$ for variations in the output gap; thus the low assumed value for $\lambda_{x}$ (relative to ad hoc loss functions often assumed in the literature on monetary policy evaluation) does not imply a low Taylor-rule response coefficient, relative to conventional recommendations. ${ }^{13}$ If, instead, one believes that a proper weight on output-gap stabilization as a policy goal requires that $\lambda_{x}$ be much higher than the value assumed here, the optimal value of $\phi_{x}$ should be correspondingly higher; see equation (1.12) below.

Probably the most important difference between this constrained-optimal rule and Taylor's is that the implicit target inflation rate here is near zero, whereas Taylor assumes a target rate of 2 percent per year. It is important also to note that the value $\phi_{x}=.57$ refers to the optimal response to fluctuations in a theoretical concept of the output gap $\left(x_{t} \equiv \hat{Y}_{t}-\hat{Y}_{t}^{e}\right)$ that may not correspond too closely to conventional "output gap" measures, which are often simply real GDP relative to some smooth trend. ${ }^{14}$ Instead, the microeconomic foundations of our model imply that the efficient level of output $\hat{Y}_{t}^{e}$ should be affected by real distur-

\footnotetext{
${ }^{12}$ While the value of $\beta$ reported in Table 1 is equal to .99, rounding to only two significant digits, and this value would imply that $\bar{\imath}$ should equal approximately .01 per quarter, 4 percent per year, the estimates of Rotemberg and Woodford (1997) actually imply a long-run average real federal funds rate closer to 3 per cent per year, so this is the value reported for $4 \bar{\imath}$ here. Because the Rotemberg-Woodford estimates imply an optimal inflation target only slightly above zero, the value of the constant term in this rule is essentially the value of the annualized interest rate consistent with zero average inflation.

${ }^{13}$ In particular, this result shows that the reason for the extremely low optimal output-response coefficients obtained in the study of Rotemberg and Woodford (1999) is not the low value of $\lambda_{x}$ assumed in that analysis; it is rather the fact that in their various families of simple policy rules, the coefficients in question indicate response to a conventional output-gap measure rather than to the welfare-relevant gap, as discussed below.

${ }^{14}$ In the case of Taylor's (1993) discussion of the degree to which his proposed rule could account for actual US policy under Greenspan's chairmanship of the Fed, the linearly detrended log of real GDP is used an empirical proxy for $x_{t}$.
} 
bances of all sorts (including some that would conventionally be classified as "demand" disturbances), and these disturbances may include some high or medium-frequency components. If one were instead to ask what the constrained-optimal rule would be within the simple family (1.5), but with $x_{t}$ replaced by detrended output $\hat{Y}_{t}$, the optimal value of the output coefficient may be quite different - it need not even be positive! For example, Gali (2000) considers this question in the context of a calibrated model similar to our baseline model, in which the real disturbances are technology shocks, and concludes that the optimal output response coefficient is zero when detrended output is used in the Taylor rule instead of the theoretically correct gap measure. Rotemberg and Woodford (1999) reach a similar conclusion (an optimal output-gap coefficient of only 0.02) in the context of their related but more complex model, with disturbance processes inferred from US time series. In the case that there are substantial deviations of the efficient level of output from a smooth trend, as both of these analyses imply, the conventional gap measure is not at all closely related to variations in the welfare-relevant gap, and a substantial positive response to it - stabilizing the conventional gap but thereby destabilizing the welfare-relevant gap, as well as inflation - can have undesirable consequences from the point of view of the welfare-theoretic stabilization goals assumed here. ${ }^{15}$

But even if the rule incorporates the correct implicit inflation target and is implemented using a correct measure of the output gap, there remain disadvantages of the Taylor rule as a policy prescription. For one, the constrained-optimality of the coefficients in (1.11) is demonstrated only in the case of a particular specification of the real disturbance processes only two disturbances, each an $\mathrm{AR}(1)$ process with a serial correlation coefficient of exactly .35. The optimal coefficients are in fact quite sensitive to the assumed degree of persistence of the disturbances; for example, in the special case that $\rho_{r}=\rho_{u}=\rho$, they are given by

$$
\phi_{\pi}=\frac{\kappa \sigma}{\lambda_{i}[(1-\rho)(1-\beta \rho)-\rho \kappa \sigma]}, \quad \phi_{x}=\frac{4 \lambda_{x} \sigma(1-\beta \rho)}{\lambda_{i}[(1-\rho)(1-\beta \rho)-\rho \kappa \sigma]} .
$$

\footnotetext{
${ }^{15}$ See also McCallum (2001) and Woodford (2001) for related discussions, with additional evidence suggesting that conventional and welfare-relevant gap measures may not be at all closely related in historical time series for the US.
} 
In the case of our calibrated values for the other parameters, this implies that the optimal coefficients take the values $\phi_{\pi}=.96, \phi_{x}=.41$ if the common $\rho$ is assumed to be as low as .17, while they instead take unboundedly large values if it is assumed to be as high as $.68{ }^{16}$ Thus this constrained-optimal rule is not robustly optimal in the sense discussed in Giannoni and Woodford (2002). This makes it an unappealing policy prescription, for in practice policymakers are not simply likely to doubt whether the assumed values of $\rho_{r}$ and $\rho_{u}$ correctly represent the typical degree of persistence of disturbances of the two types; they are instead likely to deny that all such disturbances possess any single degree of persistence, and thus to remain skeptical about the wisdom of commitment to a rule that is optimal only if all disturbances are linear combinations of only two types.

Furthermore, even if it is literally true that only two types of disturbances ever occur and they are correctly described by (1.6) - (1.7), the Taylor rule (1.11) is not a fully optimal rule. In the best case, it implements the optimal non-inertial plan, but as is shown in Woodford (1999a), this is not generally the optimal plan. It is possible to do better by committing to a rule that incorporates an appropriate form of history-dependence. As we shall see, introducing history-dependence of the right kind can eliminate both of these defects of the simple Taylor rule.

\section{$1.2 \quad$ A Robustly Optimal Instrument Rule}

We turn now to the search for a rule that can instead implement the optimal pattern of responses to real disturbances. As in Woodford (1999a), the state-contingent plan that minimizes the objective $(1.3)$ - (1.4) subject to the constraints $(1.1)-(1.2)$ satisfies the first-order conditions

$$
\pi_{t}-\beta^{-1} \sigma \Xi_{1 t-1}+\Xi_{2 t}-\Xi_{2 t-1}=0
$$

\footnotetext{
${ }^{16}$ In the case of $\rho<.17$, the coefficients given by (1.12) cease to imply a determinate equilibrium, as the "Taylor Principle" ceases to be satisfied. In the case of $\rho>.68$, the denominators of both expressions in (1.12) become negative, implying $\phi_{\pi}, \phi_{x}<0$. While these are possible rules in our discrete-time model, and even imply a determinate equilibrium as long as $\rho<.79$, the analysis for this range of parameter values takes too literally the assumption that all economic decisions are made only at discrete (quarterly) intervals, and so we choose not to emphasize the possibility of using a Taylor rule to implement the optimal non-inertial plan in this case.
} 


$$
\begin{gathered}
\lambda_{x}\left(x_{t}-x^{*}\right)+\Xi_{1 t}-\beta^{-1} \Xi_{1 t-1}-\kappa \Xi_{2 t}=0, \\
\lambda_{i}\left(i_{t}-i^{*}\right)+\sigma \Xi_{1 t}=0,
\end{gathered}
$$

for each date $t \geq 0,{ }^{17}$ together with the initial conditions

$$
\Xi_{1,-1}=\Xi_{2,-1}=0
$$

(Here $\Xi_{1 t}$ and $\Xi_{2 t}$ are the Lagrange multipliers associated with constraints (1.1) and (1.2) respectively.) In the case that a bounded optimal plan exists, it can be described by equations for $\pi_{t}, x_{t}, i_{t}, \Xi_{1 t}$ and $\Xi_{2 t}$ as linear functions of $\Xi_{1, t-1}$ and $\Xi_{2, t-1}$ together with the current and expected future values of the exogenous disturbances; these linear equations with constant coefficients apply in all periods $t \geq 0$, starting from the initial conditions (1.16).

It follows from these first-order conditions that in the case of an optimal commitment that has been in force since at least period $t-2$, it is possible to infer the values of $\Xi_{1, t-1}$ and $\Xi_{2, t-1}$ from the values that have been observed for $x_{t-1}, i_{t-1}$, and $i_{t-2}$. Specifically, one can infer the value of $\Xi_{1, t-1}$ from the value of $i_{t-1}$ using (1.15), and similarly the value of $\Xi_{1, t-2}$ from the value of $i_{t-2}$. Then substituting these values into (1.14) for period $t-1$, one can also infer the value of $\Xi_{2, t-1}$ from the value of $x_{t-1}$. One can, of course, similarly solve for the period $t$ Lagrange multipliers as functions of $x_{t}, i_{t}$, and $i_{t-1}$. Using these expressions to substitute out the Lagrange multipliers in (1.13), one obtains a linear relation among the endogenous variables $\pi_{t}, x_{t}, x_{t-1}, i_{t}, i_{t-1}$ and $i_{t-2}$ that must hold in any period $t \geq 2$. This thus provides a candidate policy rule that is consistent with the optimal state-contingent plan.

Because the relation in question involves a non-zero coefficient on $i_{t}$, it can be expressed as an implicit instrument rule of the form

$$
i_{t}=\left(1-\rho_{1}\right) i^{*}+\rho_{1} i_{t-1}+\rho_{2} \Delta i_{t-1}+\phi_{\pi} \pi_{t}+\phi_{x} \Delta x_{t} / 4
$$

where

$$
\rho_{1}=1+\frac{\kappa \sigma}{\beta}>1, \quad \rho_{2}=\beta^{-1}>1
$$

\footnotetext{
${ }^{17}$ In terms of the notation of Giannoni and Woodford (2002, sec. 5), we here assume that $t_{0}=0$.
} 


$$
\phi_{\pi}=\frac{\kappa \sigma}{\lambda_{i}}>0, \quad \phi_{x}=\frac{4 \sigma \lambda_{x}}{\lambda_{i}}>0 .
$$

We can furthermore show (see Appendix for proof) that commitment to this rule implies a determinate equilibrium.

Proposition 6. Suppose that a bounded optimal state-contingent plan exists. Then in the case of any parameter values $\sigma, \kappa, \lambda_{x}, \lambda_{i}>0$ and $0<\beta<1$, a commitment to the rule described by $(1.17)$ - (1.19) implies a determinate rational-expectations equilibrium.

The equilibrium determined by commitment to this rule from date $t=0$ onward corresponds to the unique bounded solution to equations (1.13) - (1.15) when the initial conditions (1.16) are replaced by the values of $\Xi_{1,-1}$ and $\Xi_{2,-1}$ that would be inferred from the historical values of $x_{-1}, i_{-1}$, and $i_{-2}$ under the reasoning described above.

It follows that the equilibrium determined by commitment to the time-invariant instrument rule (1.17) involves the same responses to random shocks in periods $t \geq 0$ as under the optimal commitment. This is thus an example of an instrument rule that is optimal from a timeless perspective, in the sense defined in Giannoni and Woodford (2002, sec. 3). Note that we could instead implement precisely the optimal once-and-for-all commitment from date $t=0$ onward (the bounded solution to (1.13) - (1.15) with initial conditions (1.16)) by committing to (1.17) in all periods $t \geq 2$, but to a modified version of the rule in periods $t=0$ and 1 . But this would be a non-time-invariant rule (policy would depend upon the date relative to the date at which the commitment had been made), and the preferability of this alternative equilibrium, from the standpoint of expected welfare looking forward from date $t=0$, would result from the alternative policy's optimal exploitation of prior expectations that are already given in that period. Choice of a rule that is optimal from a timeless perspective requires us to instead commit to set the interest rate according to the time-invariant rule (1.17) in all periods.

The rule (1.17) has the additional advantage of being robustly optimal, in the sense defined in Giannoni and Woodford (2002, sec. 4). We note that our derivation of the optimal 
rule has required no hypotheses about the nature of the disturbance processes $\left\{r_{t}^{n}, u_{t}\right\}$, except that they are exogenously given and that they are bounded. In fact, the rule is optimal regardless of their nature; commitment to this rule implies the optimal impulse responses displayed in Woodford (1999a) in the case of the particular disturbance processes assumed in the numerical illustrations there, but it equally implies optimal responses in the case of any other types of disturbances to the natural rate of interest and/or "cost-push shocks" disturbances that may be anticipated some quarters in advance, disturbances the effects of which do not die out monotonically with time, and so on. ${ }^{18}$ Indeed, one may assume that both of the disturbances $r_{t}^{n}$ and $u_{t}$ in equations (1.1) - (1.2) are composite disturbances of the general form discussed in Giannoni and Woodford (2002, sec. 4), and (1.17) remains an optimal rule. This robustness of the rule is a strong advantage from the point of view of its adoption as a practical guide to the conduct of monetary policy.

It is important to note that (1.17) is not a uniquely optimal instrument rule; it is not even the only rule that is robustly optimal in the sense just discussed. For example, other rules that are equally consistent with the optimal responses to disturbances, regardless of the nature of the disturbance processes, may be obtained by substituting for variables in (1.17) using one or the other of the structural equations (1.1) - (1.2). ${ }^{19}$ However, alternative optimal rules derived in this way will not be direct rules, insofar as they will involve feedback from past, current, or expected future real disturbances as well as from the paths of the target variables. (One might arrange for the disturbance terms to cancel, under a particular hypothesis about the statistical properties of the disturbances, but the version of the rule

\footnotetext{
${ }^{18}$ This is a substantial advantage of this instrument rule over the one proposed in Woodford (1999a), which expresses the federal funds rate as a function of the lagged funds rate, the lagged rate of increase in the funds rate, the current inflation rate, and the previous quarter's inflation rate. That rule would also be consistent with optimal responses to real disturbances, but only if (as assumed in the earlier calculation) all disturbances perturb the natural rate of interest in a way that can be described by an AR(1) process (1.6) with a single specified coefficient of serial correlation, and have no effect on the natural rate of output that is different than the effect on the efficient rate of output (i.e., there are no cost-push shocks). In this special case, however, the rule discussed earlier has the advantage that its implementation requires no information on the part of the central bank other than an accurate measure of inflation (including an accurate projection of period $t$ inflation at the time that the period $t$ funds rate is set).

${ }^{19}$ A specific example: one might use (1.2) to substitute for $\pi_{t}$ in (1.17), and obtain a rule for setting $i_{t}$ as a function of $i_{t-1}, i_{t-2}, x_{t}, x_{t-1}, \mathrm{E}_{t} \pi_{t+1}$, and $u_{t}$.
} 
that omitted reference to the disturbances would not be robustly optimal.)

A robustly optimal direct rule must be an implication of the first-order conditions (1.13) - (1.15) only, in order for it not to refer to the structural disturbances; and in order for it not to refer to the Lagrange multipliers, either, it must in fact be an implication of (1.17). This still does not make (1.17) the unique such rule. For example, if (1.17) holds in all periods, it follows that

$$
\begin{aligned}
i_{t}=\left(1-\rho_{1}\right)\left(1-\rho_{3}\right) i^{*} & +\left[\rho_{1}\left(1-\rho_{3}\right)+\rho_{3}\right] i_{t-1}+\left(\rho_{2}+\rho_{1} \rho_{3}\right) \Delta i_{t-1} \\
& -\rho_{2} \rho_{3} \Delta i_{t-2}+q_{t}-\rho_{3} q_{t-1}
\end{aligned}
$$

must also hold in all periods, where

$$
q_{t} \equiv \phi_{\pi} \pi_{t}+\left(\phi_{x} / 4\right) \Delta x_{t}
$$

and $\rho_{3}$ is an arbitrary coefficient. (This relation is obtained from (1.17) by adding to the right-hand side $\rho_{3}$ times $i_{t-1}$ minus the right-hand side at date $t-1$.) Condition (1.20) can also be interpreted as a direct implicit instrument rule, and it too is consistent with the optimal responses to all real disturbances, regardless of the statistical properties of those disturbances. Since we know that the rule implies a determinate equilibrium when $\rho_{3}=0$, it follows by continuity that it will also imply a determinate equilibrium for all small enough $\rho_{3} \neq 0$. Hence there exist rules of this form that are also robustly optimal direct instrument rules. But the additional history-dependence introduced into (1.20) is unnecessary; (1.17) is unambiguously a simpler rule. The same objection may be raised against the rules with additional lead terms that can be derived from (1.17) by substituting for some terms using the conditional expectation at date $t$ of both sides of (1.17) at some future date.

Another relation implied by (1.17) that does not involve a larger number of terms is

$$
i_{t}=\left(\rho_{1}+\rho_{2}\right)^{-1}\left[\left(\rho_{1}-1\right) i^{*}+\mathrm{E}_{t} i_{t+1}+\rho_{2} i_{t-1}-E_{t} q_{t+1}\right] .
$$

(This relation is equivalent to the statement that (1.17) holds at date $t+1$ only in expectation conditional upon public information at date $t$.) This too might conceivably be interpreted as 
an implicit instrument rule for setting $i_{t}$ at date $t$, though in this case a forecast-based rule. However, while this relation is consistent with the optimal responses to disturbances, imposition of (1.22) as a monetary policy rule does not determine a unique rational-expectations equilibrium. ${ }^{20}$ Thus (1.22) — which is implied by but does not imply (1.17) — does not represent a completely specified monetary policy rule under the criterion proposed in Giannoni and Woodford (2002, sec. 2), for it does not imply a determinate state-contingent path for the central bank's policy instrument. The same is true a fortiori of the relation that would be obtained from the conditional expectation at $t$ of (1.17) at $t+2$. Thus we conclude that (1.17) is of unique interest as the simplest possible robustly optimal direct instrument rule, in the case of our basic neo-Wicksellian model.

The optimal rule (1.17) has a number of important similarities to the Taylor rule. Like the Taylor rule, (1.17) is an example of a direct, implicit instrument rule. The rule is also similar to Taylor's recommendation in that the contemporaneous effect of an increase in either inflation or the output gap upon the federal funds rate operating target is positive $\left(\phi_{\pi}, \phi_{x}>0\right)$; and the rule satisfies the "Taylor principle," given that $\phi_{\pi}>0$ and $\rho_{1}>1 .{ }^{21}$ However, this optimal rule involves additional history-dependence, owing to the non-zero weights on the lagged funds rate, the lagged rate of increase in the funds rate, and the lagged output gap. And the optimal degree of history-dependence is non-trivial: the optimal values of $\rho_{1}$ and $\rho_{2}$ are both necessarily greater than one, while the optimal coefficient on $x_{t-1}$ is as large (in absolute value) as the coefficient on $x_{t}$. It is particularly worth noting that the optimal rule implies not only intrinsic inertia in the dynamics of the funds rate - a transitory deviation of the inflation rate from its average value increases the funds rate not only in the current quarter, but in subsequent quarters as well — but is actually super-inertial: the

\footnotetext{
${ }^{20}$ We can easily see this by noting that $(1.22)$ makes $i_{t}$ a function of no predetermined state variables other than $i_{t-1}$. Hence if this rule did imply a determinate equilibrium, in that equilibrium, $\pi_{t}, x_{t}$ and $i_{t}$ would all be linear functions of $i_{t-1}$ and the exogenous states that suffice to forecast the real disturbances from period $t$ onward. Yet we know that the optimal responses to shocks generally involve more complex dependence upon history than can be summarized by a single predetermined variable such as $i_{t-1}$; for one cannot generally infer the values of both $\Xi_{1, t-1}$ and $\Xi_{2, t-1}$ from the value of $i_{t-1}$ alone. We thus show by contradiction that (1.22) cannot imply a determinate equilibrium.

${ }^{21}$ See the discussion in Woodford (2002, chap. 4, sec. 2.2) of the generalization of this principle to the case of policy rules with interest-rate inertia.
} 
implied dynamics for the funds rate are explosive, ${ }^{22}$ if the initial overshooting of the longrun average inflation rate is not offset by a subsequent undershooting (as actually always happens, in equilibrium). In this respect this optimal rule is similar to those found to be optimal in the numerical analysis by Rotemberg and Woodford (1999) of a more complicated empirical version of the model.

In the case of the calibrated parameter values in Table 1 below, the coefficients of the optimal instrument rule are given by $\rho_{1}=1.15, \rho_{2}=1.01, \phi_{\pi}=.64$, and $\phi_{x}=.33$. These may be compared with the coefficients of the Fed reaction function of similar form estimated by Judd and Rudebusch (1998) for the Greenspan period: $\rho_{1}=.73, \rho_{2}=.43, \phi_{\pi}=.42$, and $\phi_{x}=.30$, except in this empirical reaction function $\phi_{x}$ represents the reaction to the current quarter's level of the output gap, rather than its first difference. ${ }^{23}$ (Interestingly, they find that an equation with feedback from the first difference of the output gap, rather than its level, fits best during an earlier period of Fed policy, under Paul Volcker's chairmanship.) The signs of the coefficients of the optimal rule agree with those characterizing actual policy; in particular, the estimated reaction function includes substantial positive coefficients $\rho_{1}$ and $\rho_{2}$, though these are still not as large as the optimal values. Thus the way in which actual Fed policy is more complex than adherence to a simple Taylor rule can largely be justified as movement in the direction of optimal policy, according to the simple model of the transmission mechanism assumed here.

We find that in the case of this simple model at least, it is not necessary for the central bank's operating target for the overnight interest rate to respond to forecasts of the future evolution of inflation or of the output gap in order for policy to be fully optimal — and not just optimal in the case of particular assumed stochastic processes for the disturbances, but

\footnotetext{
${ }^{22}$ Technically, this corresponds to the observation that in the equivalent representation (1.23) of the policy rule given below, there exists a root $\lambda_{2}>1$. A sufficient condition for this is that $\rho_{1}>1$, in which case exactly one of the roots is greater than 1.

${ }^{23}$ It should also be noted that the output gap measure used in Judd and Rudebusch's empirical analysis, while a plausible measure of what the Fed is likely to have responded to, may not correspond to the welfarerelevant output gap indicated by the variable $x_{t}$ in the optimal rule (1.17). In addition, $\phi_{\pi}$ indicates response to the most recent four-quarter growth in the GDP deflator, rather than an annualized inflation rate over the past quarter alone.
} 
robustly optimal. Thus the mere fact that the central bank may sometimes have information about future disturbances, that are not in any way disturbing demand or supply conditions yet, is not a reason for feedback from current and past values of the target variables to be insufficient as a basis for optimal policy. This does not mean that it may not be desirable for monetary policy to restrain spending and/or price increases even before the anticipated real disturbances actually take effect. But in the context of a forward-looking model of private-sector behavior, a commitment to respond to fluctuations in the target variables only contemporaneously and later does not preclude effective pre-emptive constraint of that kind. First of all, such a policy may well mean that the central bank does adjust its policy instrument immediately in response to the news, insofar as forward-looking private-sector behavior may result in an immediate effect of the news upon current inflation and output. ${ }^{24}$ And more importantly, in the presence of forward-looking private-sector behavior, the central bank mainly affects the economy through changes in expectations about the future path of its instrument in any event; a predictable adjustment of interest rates later, once the disturbances substantially affect inflation and output, should be just as effective in restraining private-sector spending and pricing decisions as a preemptive increase in overnight interest rates immediately.

At the same time, it is important to note that the optimal rule (1.17), while not "forecastbased" in the sense in which this term is usually understood, does depend upon projections of inflation and output in the same quarter as the one for which the operating target is being set. Thus the rule is not an explicit instrument rule in the sense of Svensson and Woodford (1999). And this implicit character (a feature that it shares with the Taylor rule) is crucial to the optimality of the rule, at least if we wish to find an optimal rule that is also a direct rule (specifying feedback only from the target variables). For optimal policy must generally involve an immediate adjustment of the short-term nominal interest rate in response to shocks, as shown in Woodford (1999a); ${ }^{25}$ and so unless the rule is to be specified in terms of

\footnotetext{
${ }^{24}$ This is obviously not the case if, as more realistic models often assume, there are delays in the effect of any new information on prices and spending. But in this case, it is probably not desirable for overnight interest rates to respond immediately to news, either; see section 3.2 below.
} 
the central bank's response to particular shocks, it will have to specify a contemporaneous response to fluctuations in the target variables, and not simply a lagged response. Thus implementation of such a rule will involve judgment of some sophistication about current conditions; it cannot be implemented mechanically on the basis of a small number of publicly available statistics.

\subsection{A Robustly Optimal Targeting Rule}

We have just seen that policy need not be forecast-based in order to be optimal, and even robustly optimal. At the same time, this does not mean that a forecast-based decision procedure cannot have equally desirable properties. In fact, the policy rule just discussed is equivalent, in a certain sense, to a forecast-based rule. This alternative representation of optimal policy is also of interest as an example of a robustly optimal policy rule that takes the form of a (pure) targeting rule, rather than as an expression that presents, even implicitly, a formula for the bank's interest-rate operating target. ${ }^{26}$

We can write the implicit instrument rule (1.17) in the form

$$
\left(1-\lambda_{1} L\right)\left(1-\lambda_{2} L\right) \hat{\imath}_{t}=\hat{q}_{t}
$$

where $\hat{\imath}_{t} \equiv i_{t}-\bar{\imath}$, and $\hat{q}_{t}$ similarly denotes the deviation of $q_{t}$ (the function of the target variables to which the central bank responds, defined in (1.21)) from its long-run average value, $\phi_{\pi} \bar{\pi}$. Because the optimal coefficients (1.18) are such that $\rho_{1}>1, \rho_{2}>0$, the roots in the factorization (1.23) necessarily satisfy $0<\lambda_{1}<1<\lambda_{2}$. It then follows that relation

\footnotetext{
${ }^{25}$ This is not true if there are delays in the effects of shocks upon inflation and output, as discussed in section 3.2 below. But in that case, even the delayed effect upon the central bank's instrument that is required by optimal policy cannot be implemented on the basis only of lagged observations of the target variables, because of the delay with which shocks affect these variables.

${ }^{26}$ Svensson (1999) uses the concept of a "targeting rule" somewhat more broadly, to include the possibility of target criteria which may or may not depend on interest rates. Hence there is no sharp distinction between targeting rules and implicit instrument rules of the kind discussed in the previous section. We find it of interest, however, that optimal policy admits of alternative representations in this case, in only one of which the rule can be written as an implicit instrument rule; in the case that no such interpretation is possible, we refer to a pure targeting rule. The two alternative representations of optimal policy can also be distinguished in terms of the degree of forward integration of the target criterion.
} 
(1.17) is equivalent to the relation

$$
\left(1-\lambda_{1} L\right) \hat{\imath}_{t-1}=-\lambda_{2}^{-1} \mathrm{E}_{t}\left[\left(1-\lambda_{2}^{-1} L^{-1}\right)^{-1} \hat{q}_{t}\right]
$$

in the following sense.

Proposition 7. Two bounded stochastic processes $\left\{\hat{\imath}_{t}, \hat{q}_{t}\right\}$ satisfy (1.23) for all $t \geq 0$ if and only if they satisfy (1.24) for all $t \geq 0$.

(See proof in the Appendix.)

Thus there is no difference between the way in which a central bank must adjust its interest-rate instrument to ensure that (1.17) holds in all periods and the way that it would adjust it to ensure that (1.24) holds in all periods, for the two conditions imply one another. (This does not mean that arranging for (1.24) to hold in a single period $t$ is equivalent to arranging for (1.17) to hold in that single period, regardless of how policy is expected to be conducted thereafter; but a permanent commitment to either rule from some date $t_{0}$ onward has identical consequences.) This equivalence does not apply only in the case of processes that are possible equilibria of the model consisting of structural equations (1.1) - (1.2); thus the rules are equivalent regardless of whether that model is correctly specified, and regardless of whether the central bank expects the economy to actually evolve according to a rational-expectations equilibrium of that model or not (for example, regardless of whether the private sector is believed to correctly understand the bank's policy rule or not).

It follows from this equivalence that a commitment to ensure that (1.24) holds in all periods from some date onward represents a coherent complete specification of a monetary policy rule, at least in the context of the model described by equations (1.1) - (1.2). Hence this represents a well-defined targeting rule, even though the criterion (1.22) cannot be solved by itself to yield even an implicit expression for the period $t$ instrument setting: the left-hand side involves only lagged interest rates, while the right-hand side refers only to the evolution of inflation and the output gap. A model of the monetary transmission mechanism must be used in order to determine the instrument setting that is consistent with a projection that 
satisfies the target criterion. ${ }^{27}$

The target criterion (1.24) can be expressed in the form

$$
F_{t}(\pi)+\frac{\phi_{x}}{4} F_{t}(x)=\frac{\theta_{x}}{4} x_{t-1}-\theta_{i}\left(i_{t-1}-i^{*}\right)-\theta_{\Delta} \Delta i_{t-1}
$$

where for each of the variables $z=\pi, x$ we use the notation $F_{t}(z)$ for a conditional forecast

$$
F_{t}(z) \equiv \sum_{j=0}^{\infty} \alpha_{z, j} \mathrm{E}_{t} z_{t+j}
$$

involving weights $\left\{\alpha_{z, j}\right\}$ that sum to one. Thus the criterion specifies a time-varying target value for a weighted average of an inflation forecast and an output-gap forecast, where each of these forecasts is in fact a weighted average of forecasts at various horizons, rather than a projection for a specific future date. The rule represents a variant of what Svensson (1999) calls "flexible inflation targeting," though this rule differs from simple versions that Svensson discusses in the history-dependence of the inflation-forecast target (indicated by the non-constant terms on the right-hand side).

In representation (1.25) of this policy rule, there is no constant term, indicating an inflation-forecast target of zero except insofar as this is corrected in response to deviations (past or projected) of the output gap and/or the nominal interest rate from their target values. $^{28}$ The optimal coefficients indicating the degree to which the inflation-forecast target is adjusted are given by

$$
\begin{gathered}
\phi_{x}=\theta_{x}=4\left(1-\lambda_{2}^{-1}\right) \frac{\lambda_{x}}{\kappa}>0, \\
\theta_{i}=\lambda_{2}\left(1-\lambda_{1}\right)\left(1-\lambda_{2}^{-1}\right) \frac{\lambda_{i}}{\kappa \sigma}>0, \\
\theta_{\Delta}=\lambda_{1} \lambda_{2}\left(1-\lambda_{2}^{-1}\right) \frac{\lambda_{i}}{\kappa \sigma}>0,
\end{gathered}
$$

\footnotetext{
${ }^{27}$ Note, however, that the situation is not really different in the case of a commitment to ensure that (1.17) is satisfied: a model is still needed to determine the instrument setting that should result in current period inflation and output that imply that the implicit instrument rule is satisfied.

${ }^{28}$ Note, however, that this does not mean that the rule sets the inflation forecast equal to zero on average. This is because the target interest rate $i^{*}$ is in general not consistent with an average inflation rate of zero.
} 
while the optimal weights in the conditional forecasts are

$$
\alpha_{\pi, j}=\alpha_{x, j}=\left(1-\lambda_{2}^{-1}\right) \lambda_{2}^{-j}
$$

Thus the optimal conditional forecast is one that places positive weight on the projection for each future period, beginning with the current period, with weights that decline exponentially as the horizon increases. The mean distance in the future of the projections that are relevant to the target criterion is equal to

$$
\sum_{j=0}^{\infty} \alpha_{z, j} j=\left(\lambda_{2}-1\right)^{-1}
$$

for both the inflation and output-gap forecasts.

In the case of the calibrated parameter values reported in Table 1, the rate at which these weights decay per quarter is $\lambda_{2}^{-1}=.68$, so that the mean forecast horizon in the optimal target criterion is 2.1 quarters. Thus while our optimal example of a pure targeting rule can be expressed in terms of a target for inflation and output-gap forecasts, the forecast horizon involved is short compared to those typically considered in the recent literature, or those typical of the actual practice of inflation forecast-targeting central banks. For these same parameter values, the optimal relative weight on the output-gap forecast is $\phi_{x}=.15$, indicating that the target criterion is essentially an inflation-forecast target, albeit a modified one. The direction of modification is the one suggested by Svensson $(1997,1999)$ : a forecast of a lower output gap than normal should cause the central bank to tolerate a higher than average inflation forecast. Finally, the remaining optimal coefficients are $\theta_{x}=.15, \theta_{i}=.24$, and $\theta_{\Delta}=.51$, indicating a substantial degree of history-dependence of the optimal modified inflation-forecast target. The fact that $\theta_{x}=\phi_{x}$ indicates that it is really the forecasted increase in the output gap relative to the previous quarter's level, rather than the absolute level of the gap, that should modify the inflation-forecast target. The signs of $\theta_{i}$ and $\theta_{\Delta}$ imply that policy will be made tighter (in the sense of demanding a lower modified inflation forecast) when interest rates have been high and/or increasing in the recent past; this is another way of committing to interest-rate inertia of the kind discussed above. 
The equivalence expressed in Proposition 7 implies that commitment to a history-dependent modified inflation-forecast target of this kind is a robustly optimal policy rule in exactly the same sense as the instrument rule (1.17). Thus commitment to a pure targeting rule can be a sound approach to policy. This alternative, forward-integrated representation of optimal policy has the possible advantage (from the point of view of successfully steering private-sector expectations) of emphasizing the way in which the outlook for inflation and the output gap are adjusted at each point in time (at least as far as the intentions of the central bank are concerned) in response to variations in the recent evolution of the target variables. While this is implied by a commitment to implement the instrument rule (1.17) from now on, it might not be clear to the private sector - for example, because the central bank's commitment to continue to implement the instrument rule in the future might not be clear. Hence communication with the public about current policy decisions in terms of their implications for inflation and output-gap forecasts might be a superior way of conveying the central bank's commitments with regard to subsequent developments.

The representation of optimal policy in terms of a pure targeting rule also has the advantage of continuing to be possible even in the limiting case that $\lambda_{i}=0$, i.e., even when reducing the variability of interest rates is not an independent concern. ${ }^{29}$ In that limit, the weights $\phi_{\pi}$ and $\phi_{x}$ in (1.17) become unboundedly large, so that a representation of optimal policy in terms of a direct instrument rule ceases to be possible. Instead, the coefficients of (1.25) remain well-defined: $\theta_{i}$ and $\theta_{\Delta}$ become equal to zero, while $\phi_{x}, \theta_{x}$, and the weights $\left\{\alpha_{z, j}\right\}$ continue to take well-defined positive values. Thus in this limiting case, the optimal targeting rule is one in which the inflation-forecast target must be modified in proportion to the projected change in the output gap, but it is no longer also dependent on lagged interest rates.

\footnotetext{
${ }^{29}$ Svensson and Woodford (1999) consider a model closely related to this one, but assume a stabilization objective in which $\lambda_{i}=0$. It is for this reason that they find that it is possible to formulate a robustly optimal targeting rule, a forward-looking variant of (1.26) below, but not a robustly optimal instrument rule.
} 
In fact, the optimal target criterion in this case can be written more simply as

$$
\pi_{t}+\frac{\phi_{x}}{4} \Delta x_{t}=0
$$

or even in terms of a flexible price-level target

$$
\log P_{t}=\log P^{*}-\frac{\phi_{x}}{4} x_{t}
$$

where $P^{*}$ is a target price level. ${ }^{30}$ Thus optimal policy in this can be implemented by commitment to a target of the sort proposed by Hall (1984), though the optimal weight $\phi_{x}$ implied by our theory is considerably smaller (for any plausible calibration of the model's parameters) than the values suggested by Hall. Furthermore, such a policy may be implemented through a forecast-targeting procedure of the kind practiced by inflation-targeting central banks, rather than necessarily requiring institution of the sort of automatic mechanism proposed by Hall.

\section{Optimal Rules for a Model with Inflation Inertia}

The basic model considered above is often criticized as being excessively forward-looking, particularly in its neglect of any sources of intrinsic inertia in the dynamics of inflation. It might be suspected that this feature of the model is responsible for our strong conclusion above, according to which a robustly optimal policy rule need involve no dependence upon forecasts of the target variables beyond the current period. In Svensson's (1997) classic argument for the optimality of inflation-forecast targeting, it is the existence of lags in the effect of monetary policy on inflation that causes the optimal rule to involve a target criterion for a forecast, with the optimal forecast horizon coinciding with the length of the policy transmission lag. It might reasonably be suspected that forecasts are not necessary in our analysis above because our simple model includes no lags in the effects of policy.

\footnotetext{
${ }^{30}$ See Woodford (1999b) for further discussion. Even when $\lambda_{i}>0$, "Wicksellian" rules, in which the nominal interest rate is adjusted in response to deviations of the price level from a deterministic target path, rather than to deviations of inflation from a target rate as in the Taylor rule, maybe be desirable by comparison with other equally simple rules, as shown by Giannoni (2001).
} 
Here we take up this question by extending our analysis to the case of the model of inflation inertia developed in Woodford (2002, chap. 3). In this extension of our basic model, prices are not held constant between the dates at which they are re-optimized, but instead are automatically adjusted on the basis of the most recent quarter's increase in the aggregate price index, by a percentage that is a fraction $\gamma$ of the percentage increase in the index. $^{31}$ The aggregate supply relation (1.2) then takes the more general form

$$
\pi_{t}-\gamma \pi_{t-1}=\kappa x_{t}+\beta \mathrm{E}_{t}\left(\pi_{t+1}-\gamma \pi_{t}\right)+u_{t}
$$

where the coefficient $\kappa$ and the disturbance $u_{t}$ are defined as before. For $\gamma$ substantially greater than zero, this makes past inflation an important determinant of current inflation, along with current and expected future output gaps and cost-push shocks; if $\gamma$ is close enough to one, even a monetary disturbance that has only a transitory effect on real activity can have a much longer-lasting effect on inflation.

The aggregate-demand side of our model remains as before, and our model can accordingly be summarized by the two structural equations (1.1) and (2.1), together with exogenous stochastic processes for the disturbances $\left\{r_{t}^{n}, u_{t}\right\}$. As shown in Woodford (2002, chap. 6), the change in our assumptions about pricing behavior implies a corresponding change in the appropriate welfare-theoretic stabilization objective for monetary policy. This is once again a discounted criterion of the form (1.3), but the period loss function becomes

$$
L_{t}=\left(\pi_{t}-\gamma \pi_{t-1}\right)^{2}+\lambda_{x}\left(x_{t}-x^{*}\right)^{2}+\lambda_{i}\left(i_{t}-i^{*}\right)^{2}
$$

We wish to consider policies that minimize the criterion defined by (1.3) and (2.2), subject to the constraints imposed by the structural equations (1.1) and (2.1), for arbitrary values of the indexation parameter $0 \leq \gamma \leq 1$. $^{32}$

\footnotetext{
${ }^{31}$ Indexation of this kind was first proposed by Christiano et al. (2001), and has since been incorporated into estimated models of wage and price dynamics by Altig et al. (2002), Smets and Wouters (2002), and Sbordone (2002b).

${ }^{32} \mathrm{An}$ alternative way of modeling inflation inertia would be to assume the existence of backward-looking "rule of thumb" price-setters, as in Gali and Gertler (1999). This leads to a modification of the aggregate supply relation that is similar, though not quite identical, to (2.1). Steinsson (2000) and Amato and Laubach
} 


\subsection{Robustly Optimal Instrument Rules}

In the case of this generalization of our policy problem, the first-order condition (1.13) becomes instead

$$
\pi_{t}^{q d}-\beta \gamma \mathrm{E}_{t} \pi_{t+1}^{q d}-\beta^{-1} \sigma \Xi_{1 t-1}-\beta \gamma \mathrm{E}_{t} \Xi_{2, t+1}+(1+\beta \gamma) \Xi_{2 t}-\Xi_{2 t-1}=0,
$$

where

$$
\pi_{t}^{q d} \equiv \pi_{t}-\gamma \pi_{t-1}
$$

is the quasi-differenced inflation rate that appears in both the aggregate supply relation (2.1) and the loss function (2.2). Conditions (1.14) - (1.15) remain as before, and this system of three equations, together with initial conditions (1.16) and an initial condition for $\pi_{-1}$, continues to define the optimal once-and-for-all commitment to apply from date $t=0$ onward.

As above, we can use conditions (1.14) - (1.15) to substitute for $\Xi_{1 t}$ and $\Xi_{2 t}$ in (2.3), obtaining an Euler equation of the form

$$
\mathrm{E}_{t}\left[A(L)\left(i_{t+1}-i^{*}\right)\right]=-f_{t}
$$

for the optimal evolution of the target variables. Here $A(L)$ is a cubic lag polynomial

$$
A(L) \equiv \beta \gamma-(1+\gamma+\beta \gamma) L+\left(1+\gamma+\beta^{-1}(1+\kappa \sigma)\right) L^{2}-\beta^{-1} L^{3},
$$

while the term $f_{t}$ is a function of the observed and expected future paths of the target variables, defined by

$$
\begin{aligned}
f_{t} & \equiv \tilde{q}_{t}-\beta \gamma \mathrm{E}_{t} \tilde{q}_{t+1}, \\
\tilde{q}_{t} & \equiv \frac{\kappa \sigma}{\lambda_{i}}\left[\pi_{t}^{q d}+\frac{\lambda_{x}}{\kappa} \Delta x_{t}\right] .
\end{aligned}
$$

(Note that the above definition generalizes the earlier (1.21), and that in the limit where $\gamma=0, f_{t}$ is equal to $\tilde{q}_{t}$, which equals $q_{t}$.)

(2001b) derive welfare-theoretic loss functions for this model, and find that the loss each period is a quadratic function of both $\pi_{t}$ and $\pi_{t-1}$ that is similar, though again not identical, to our loss function (2.2). Hence we conjecture that similar conclusions as to the degree to which optimal policy is forward-looking would be obtained using the Gali-Gertler model, though we do not take this up here. 
By an argument directly analogous to the proof of Proposition 6, we can show that if a bounded optimal state-contingent plan exists, the system obtained by adjoining (2.5) to the structural equations (1.1) and (2.1) implies a determinate rational-expectations equilibrium, in which the responses to exogenous disturbances are the same as under the optimal commitment. (The only difference between this equilibrium and the optimal once-and-for-all commitment just defined relates to the initial conditions, as in our earlier discussion, and once again this difference is irrelevant to the design of a policy rule that is optimal from a timeless perspective.) Hence we could regard (2.5) as implicitly defining a policy rule, and the rule would once again be robustly optimal. In the limiting case that $\gamma=0,(2.5)$ ceases to involve any dependence upon $\mathrm{E}_{t} i_{t+1}$, and the proposed rule would coincide with the optimal instrument rule (1.17) discussed above.

However, (2.5) is an even less explicit expression for the central bank's interest-rate policy than the implicit instrument rules considered earlier, for (when $\gamma>0$ ) it defines $i_{t}$ only as a function of $\mathrm{E}_{t} i_{t+1}$. This means that the central bank defines the way in which it is committed to set its instrument only as a function of the way that it expects to act further in the future. This failure to express the rule in "closed form" is especially undesirable from the point of view of our question about the optimal forecast horizon for a monetary policy rule. Expression (2.5) involves no conditional expectations for variables at dates more than one period in the future. However, this does not really mean that the central bank's forecasts for later dates are irrelevant when setting $i_{t}$. For this "rule" directs the bank to set $i_{t}$ as a function of its forecast of $i_{t+1}$, and (if the same rule is expected to be used to set $i_{t+1}$ ) the bank's forecast at $t$ of $i_{t+1}$ should involve its forecast at $t$ of $\tilde{q}_{t+2}$. It should also involve its forecast of $i_{t+2}$, and hence (by similar reasoning) its forecast of $\tilde{q}_{t+3}$, and so on. Hence it is more revealing to describe the proposed policy rule in a form that eliminates any reference to the future path of interest rates themselves, and instead refers only to the bank's projections of the future paths of inflation and the output gap. ${ }^{33}$

\footnotetext{
${ }^{33} \mathrm{~A}$ rule expressed in this way will also conform better to the evident preference of central bank's to justify their monetary policy decisions to the public in terms of their projections for the future paths of inflation and output, rather than in terms of their assumptions about the future path of interest rates.
} 
To obtain an equivalent policy rule of the desired form, we need to partially "solve forward" equation (2.5). This requires factorization of the lag polynomial as

$$
A(L) \equiv \beta \gamma\left(1-\lambda_{1} L\right)\left(1-\lambda_{2} L\right)\left(1-\lambda_{3} L\right)
$$

We note the following properties of the roots of the associated characteristic equation.

Proposition 8. Suppose that $\sigma, \kappa>0,0<\beta<1$, and $0<\gamma \leq 1$. Then in the factorization (2.9) of the polynomial defined in (2.6), there is necessarily one real root $0<$ $\lambda_{1}<1$, and two roots outside the unit circle. The latter two roots are either two real roots $\lambda_{3} \geq \lambda_{2}>1$, or a complex pair $\lambda_{2}, \lambda_{3}$ of roots with real part greater than 1 . Three real roots necessarily exist for all small enough $\gamma>0$, while a complex pair necessarily exists for all $\gamma$ close enough to 1.

(See proof in the Appendix.) We use the conventions in the statement of this proposition in referring to the distinct roots in what follows. It is also useful to rewrite (2.5) as

$$
\mathrm{E}_{t}\left[A(L) \hat{\imath}_{t+1}\right]=-\hat{f}_{t},
$$

where once again hats denote the deviations of the original variables from the long-run average values implied by the policy rule (2.5), or equivalently, by the optimal commitment.

In the case that three real roots exist, the existence of two distinct roots greater than one allows us two distinct ways of "solving forward", resulting in two alternative relations,

$$
\left(1-\lambda_{1} L\right)\left(1-\lambda_{2} L\right) \hat{\imath}_{t}=\left(\beta \gamma \lambda_{3}\right)^{-1} \mathrm{E}_{t}\left[\left(1-\lambda_{3}^{-1} L^{-1}\right)^{-1} \hat{f}_{t}\right],
$$

Public communications such as the Bank of England's Inflation Report put projections for both inflation and output at center stage, while being careful not to express any opinion whatsoever about the likely path of interest rates over the period under discussion. The forecast-based rules proposed below still refer to forecast paths conditional upon intended policy, rather than upon "constant-interest-rate" forecasts, and so it will not be possible to implement these rules without taking a stand (at least for internal purposes) on the likely future path of interest rates. But the rules make it possible to discuss the way in which the current instrument setting is required by the bank's inflation and output projections, without also discussing the interest-rate path that is implicit in those projections, and to this extent they require a less radical modification of current procedures. 
or

$$
\left(1-\lambda_{1} L\right)\left(1-\lambda_{3} L\right) \hat{\imath}_{t}=\left(\beta \gamma \lambda_{2}\right)^{-1} \mathrm{E}_{t}\left[\left(1-\lambda_{2}^{-1} L^{-1}\right)^{-1} \hat{f}_{t}\right]
$$

We can also derive other relations of the same form by taking linear combinations of these ones. Of special interest is the relation

$$
\begin{aligned}
\left(1-\lambda_{1} L\right)\left(1-\frac{\lambda_{2}+\lambda_{3}}{2} L\right) \hat{\imath}_{t} & =\frac{1}{2}\left(\beta \gamma \lambda_{3}\right)^{-1} \mathrm{E}_{t}\left[\left(1-\lambda_{3}^{-1} L^{-1}\right)^{-1} \hat{f}_{t}\right] \\
& +\frac{1}{2}\left(\beta \gamma \lambda_{2}\right)^{-1} \mathrm{E}_{t}\left[\left(1-\lambda_{2}^{-1} L^{-1}\right)^{-1} \hat{f}_{t}\right]
\end{aligned}
$$

Here relations (2.11) and (2.12) are defined (with real-valued coefficients) only in the case that three real roots exist, while relation (2.13) can also be derived (and has real coefficients on all leads and lags) in the case that $\lambda_{2}, \lambda_{3}$ are a complex pair. Because $\left|\lambda_{2}\right|,\left|\lambda_{3}\right|>1$, the right-hand side of each of these expressions is well-defined, and describes a bounded stochastic process in the case of any bounded process $\left\{\hat{f}_{t}\right\}$. (In what follows, we shall refer to the three possible expressions for an optimal instrument rule presented in $(2.11)-(2.13)$ as Rule I, Rule II, and Rule III respectively.)

Each of the relations $(2.11)-(2.13)$ can be solved for $\hat{\imath}_{t}$ as a function of two of its own lags and expectations at date $t$ regarding current and future values of $\hat{f}_{t}$. These can thus be interpreted as implicit instrument rules, each of which now avoids any direct reference to the planned future path of the central bank's instrument (though assumptions about future monetary policy will be implicit in the inflation and output-gap forecasts). Each of these policy rules is equivalent to (2.5), and they are accordingly equivalent to one another, in the following sense.

Proposition 9. Under the assumptions of Proposition 8, and in the case that the factorization (2.9) involves three real roots, a pair of bounded processes $\left\{\hat{\imath}_{t}, \hat{f}_{t}\right\}$ satisfy any of the equations $(2.11),(2.12)$ or $(2.13)$ at all dates $t \geq t_{0}$ if and only if they satisfy (2.10) at all of those same dates. In the case that a complex pair exists, (2.13) is again equivalent to $(2.10)$, in the same sense. 
(See proof in Appendix.) Each of the rules thus represents a feasible specification of monetary policy in the case that its coefficients are real-valued, and when this is true it implies equilibrium responses to real disturbances that are those associated with an optimal commitment. Accordingly, each represents an optimal policy rule from a timeless perspective. (Note that although the coefficients differ, these are not really different policies. Proposition 9 implies that they involve identical actions, if the bank expects to follow one of them indefinitely, regardless of the model of the economy used to form the conditional forecasts.)

In the case that three real roots exist, we have a choice of representations of optimal policy in terms of an instrument rule, and this time we cannot choose among them on grounds of simplicity. But rule I seems particularly appealing in this case. This is the rule (among our three possibilities, or any other linear combinations of these) that puts the least weight on forecasts far in the future. It is proper to ask at what rate the weights on forecasts shrink with the forecast horizon, under the assumption that these shrink as fast as possible consistent with robust optimality of the policy rule, if we wish to determine how much forecast-dependence is necessary for robust optimality. This choice is also uniquely desirable in the sense that it remains well-defined in the limit as $\gamma$ approaches zero. In this limit, rule I reduces to

$$
\left(1-\lambda_{1} L\right)\left(1-\lambda_{2} L\right) \hat{\imath}_{t}=\hat{f}_{t}
$$

which is the optimal instrument rule (1.23) derived earlier. ${ }^{34}$ Instead, in the case of any of the other rules, the coefficients on lagged interest rates become unboundedly large as $\gamma$ approaches zero. Thus rule I is clearly the preferable specification of policy in the case of small $\gamma$. The desire for a rule that varies continuously with $\gamma$, so that uncertainty about the precise value of $\gamma$ will not imply any great uncertainty about how to proceed, then make rule I an appealing choice over the entire range of $\gamma$ for which it is defined.

One might think that the same continuity argument could instead be used to argue for

\footnotetext{
${ }^{34}$ Note that as $\gamma \rightarrow 0, \lambda_{3} \rightarrow+\infty$, while $\gamma \lambda_{3} \rightarrow \beta^{-1}$. Recall also that in this limiting case, $\hat{f}_{t}=\hat{q}_{t}$. One can show furthermore that the two smaller roots $\lambda_{1}, \lambda_{2}$ in the factorization (2.9) approach the two roots in the factorization (1.23) of our earlier quadratic lag polynomial.
} 
the choice of rule III in all cases, since this is the only one of our optimal instrument rules that continues to be defined for high values of $\gamma$. Yet the instruction to follow rule I if three real roots exist, but rule III if there is a complex pair, is also a specification that makes all coefficients of the policy rule continuous functions of $\gamma$. The reason is that as $\gamma$ passes through a critical value $\bar{\gamma}$ at which the real roots of the characteristic equation bifurcate, the two larger real roots, $\lambda_{2}$ and $\lambda_{3}$, come to exactly equal one another. When $\bar{\gamma}$ is approached from the other direction, the imaginary parts of the complex roots $\lambda_{2}$ and $\lambda_{3}$ approach zero; at the bifurcation point their common real value is the repeated real root obtained as the common limit of the two real roots from the other direction. Hence when $\gamma=\bar{\gamma}$, rules I, II, and III are all identical. There is thus no ambiguity about whether rule I or rule III should be applied in this case, and no discontinuity in the coefficients of the recommended rule as $\gamma$ approaches $\bar{\gamma}$ from either direction. At the same time, this proposal results in a rule that remains well-defined as $\gamma$ approaches zero, and for small $\gamma>0$ results in a rule that is very close to the one previously recommended for an economy with no inflation inertia.

Each of rules I, II, and III can be written in the form

$$
i_{t}=\left(1-\rho_{1}\right) i^{*}+\rho_{1} i_{t-1}+\rho_{2} \Delta i_{t-1}+\phi_{\pi} F_{t}(\pi)+\frac{\phi_{x}}{4} F_{t}(x)-\theta_{\pi} \pi_{t-1}-\frac{\theta_{x}}{4} x_{t-1},
$$

where here we have added the constant terms again to indicate the desired level of interest rates (and not just the interest rate relative to its long-run average level), and where $F_{t}(z)$ again denotes a linear combination of forecasts of the variable $z$ at various future horizons, with weights normalized to sum to one. This form of rule generalizes the specification (1.17) that suffices in the case $\gamma=0$ in two respects: the interest-rate operating target $i_{t}$ now depends upon lagged inflation in addition to the lagged variables that mattered before, and it now depends upon forecasts of inflation and the output gap in future periods, and not simply upon the projections of those variables for the current period.

Except in these respects, the coefficients are qualitatively similar to those in (1.17), as indicated by the following proposition.

Proposition 10. Under the assumptions of Proposition 8, and a loss function with 
Table 1: Calibrated parameter values for the basic neo-Wicksellian model.

\begin{tabular}{|c|c|}
\hline \multicolumn{2}{|c|}{ Structural parameters } \\
\hline$\beta$ & 0.99 \\
\hline$\sigma^{-1}$ & 0.16 \\
\hline$\kappa$ & .024 \\
\hline \multicolumn{2}{|c|}{ Shock processes } \\
\hline$\rho\left(\hat{r}^{n}\right), \rho(u)$ & 0.35 \\
\hline \multicolumn{2}{|c|}{ Loss function } \\
\hline$\lambda_{x}$ & .048 \\
\hline$\lambda_{i}$ & .236 \\
\hline
\end{tabular}

$\lambda_{x}, \lambda_{i}>0$, each of rules I, II, and III has a representation of the form (2.14) for all values of $\gamma$ for which the rule is well-defined, and in this representation,

$$
\begin{gathered}
\rho_{1}>1, \quad \rho_{2}>0, \\
0<\theta_{\pi} \leq \phi_{\pi},
\end{gathered}
$$

and

$$
0<\theta_{x}=\phi_{x} .
$$

Furthermore, for given values of the other parameters, as $\gamma \rightarrow 0$ (for rule I) the coefficient $\theta_{\pi}$ approaches zero, though $\phi_{\pi}$ approaches a positive limit; while as $\gamma \rightarrow 1$ (for rule III) the coefficients $\theta_{\pi}$ and $\phi_{\pi}$ approach the same positive limit.

(The proof is again in the Appendix.) It is especially noteworthy that once again the optimal instrument rule is superinertial. We also note that once again what should matter is the projected output gap relative to the previous quarter's output gap, rather than the absolute level of the projected gap; and once again interest rates should be increased if the gap is projected to rise. Once again a higher projected inflation rate implies that the interest rate should be increased; but now the degree to which this is true is lower if recent inflation has been high, and in the extreme case $\gamma=1$, it is only the projected inflation rate relative to the previous quarter's rate that should matter. 

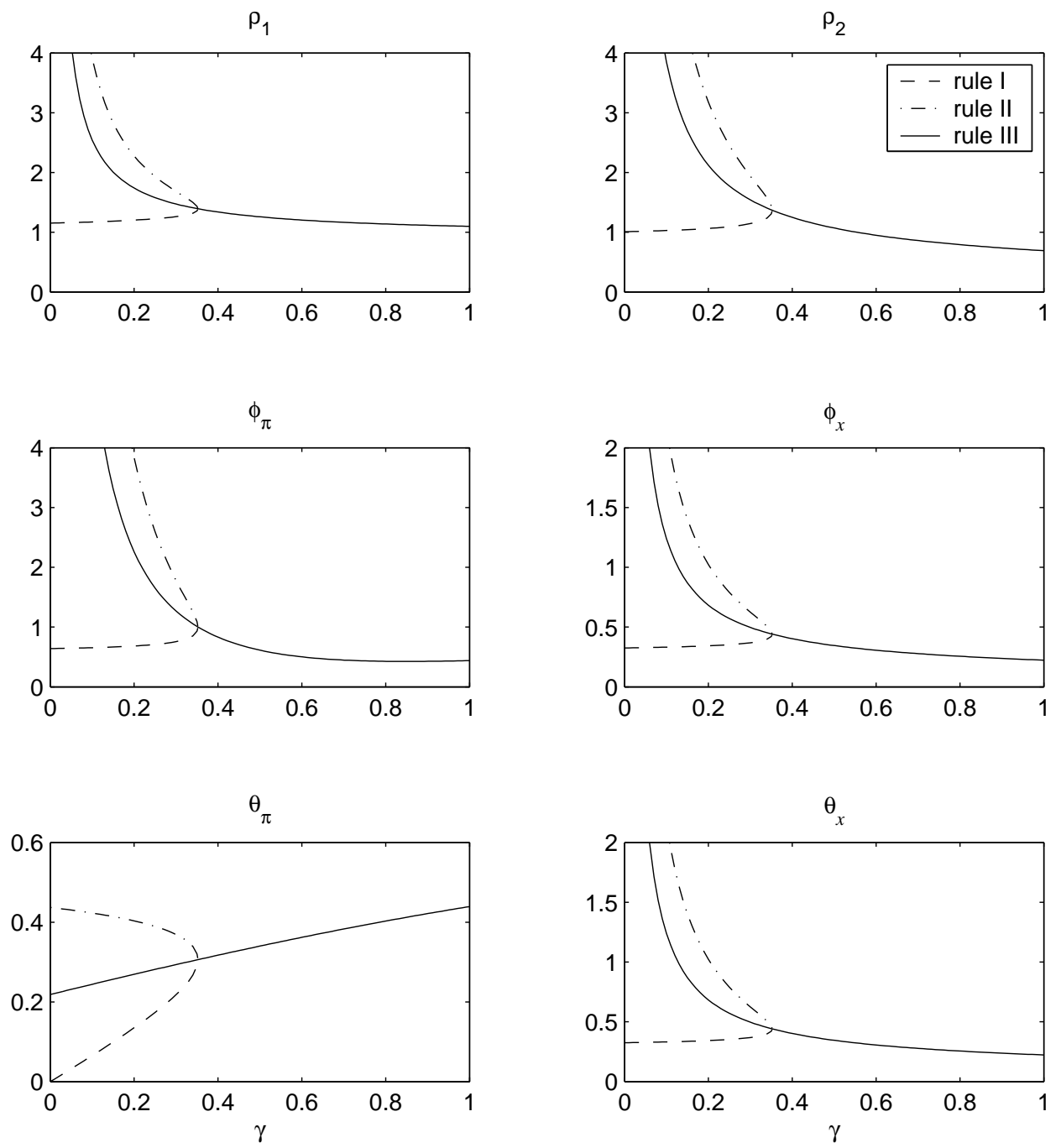

Figure 1: Coefficients of the optimal instrument rule (2.14) as functions of $\gamma$. 

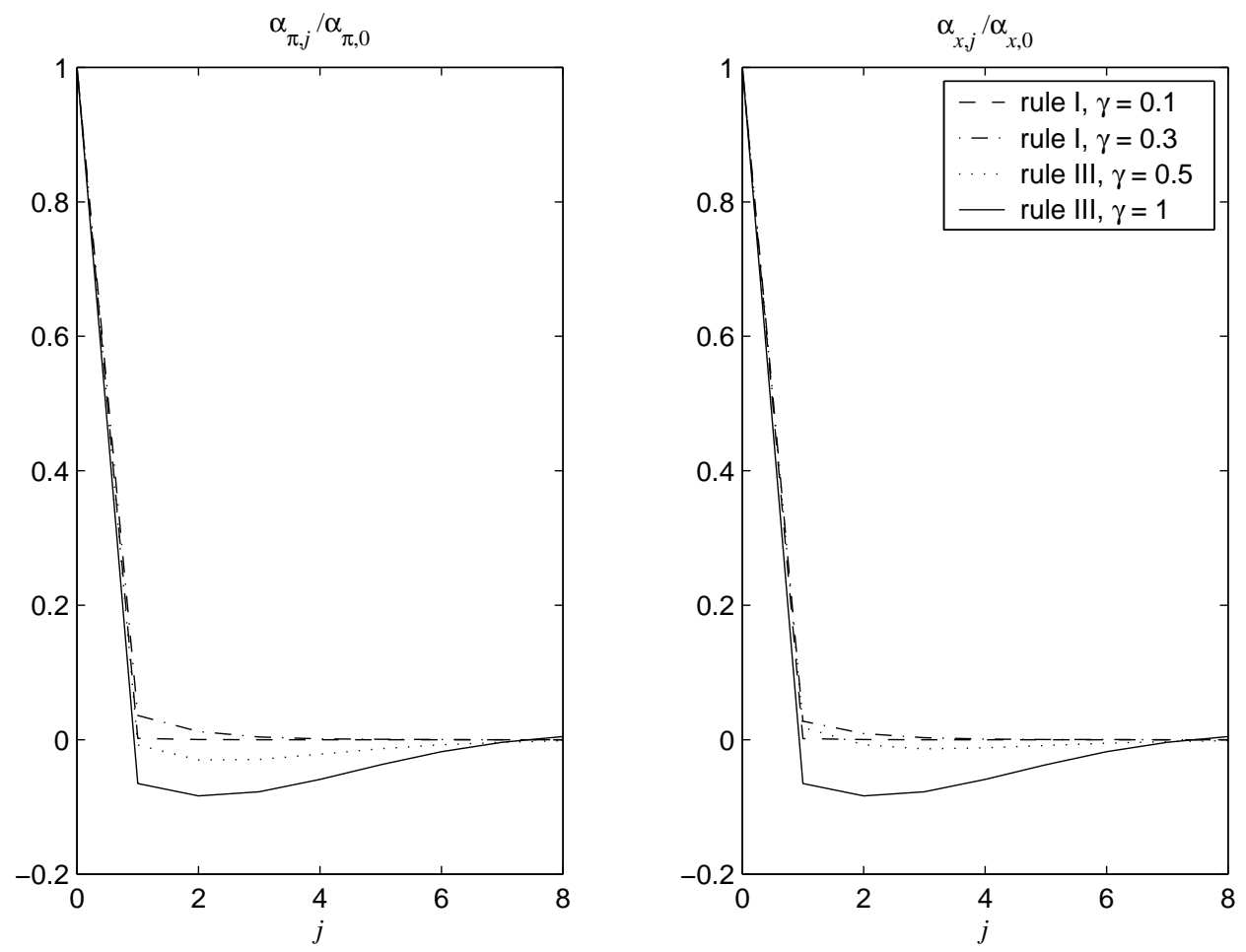

Figure 2: Relative weights on forecasts at different horizons in the optimal rule (2.14).

The numerical values of these coefficients are plotted, for alternative values of $\gamma$ ranging between zero and one, in the various panels of Figure 1, where the assumed values for the other parameters are as in Table 1 . For all values $\gamma<\bar{\gamma}=.35$, there are three real roots, and for each value of $\gamma$ the three values corresponding to rules I, II, and III are each plotted; for $\gamma>\bar{\gamma}$, only rule III is defined. An interesting feature of these plots, is that if one considers the coefficients associated with rule I for $\gamma \leq \bar{\gamma}$ and rule III for $\gamma \geq \bar{\gamma}$, one observes that the magnitude of each of the coefficients remains roughly the same, regardless of the assumed value of $\gamma$. (The exception is $\theta_{\pi}$, which approaches zero for small $\gamma$, but becomes a substantial positive coefficient for large $\gamma$, as indicated by Proposition 10.)

The panels of Figure 2 similarly plot the relative weights $\alpha_{z, j} / \alpha_{z, 0}$ for different horizons $j$ of the inflation and output-gap forecasts to which the optimal instrument rule refers, ${ }^{35}$ for

\footnotetext{
${ }^{35}$ Here we plot the relative weights, rather than the absolute weights, because this makes visual comparison between the degree of forecast-dependence of optimal policy in the different cases easier. The absolute weights can be recovered by integrating the plots shown here, since the relative weights in each case must sum to
} 
each of several different possible values of $\gamma$. (The weights associated with rule I are plotted in the case of values $\gamma<\bar{\gamma}$, and those associated with rule III in the case of values $\gamma>\bar{\gamma}$.) Here we observe that in this case the forecasts $F_{t}(z)$ are not actually weighted averages of forecasts at different horizons, because the weights are not all non-negative. Thus while in the presence of inflation inertia, the optimal instrument rule is to some extent forecastbased, the optimal responses to forecasts of future inflation and output gaps are not of the sort generally assumed in forward-looking variants of the Taylor rule. In the case of high $\gamma$, a higher forecasted inflation rate (or output gap) in any of the next several quarters implies, for given past and projected current conditions, that a lower current interest rate is appropriate. According to the optimal rule, a higher current inflation rate should be tolerated in the case that high inflation is forecast for the next several quarters. This is because (in an economy with $\gamma$ near one) it is sudden changes in the inflation rate that creates the greatest distortions in the economy, by making automatic adjustment of prices in response to lagged inflation a poor rule of thumb.

In addition to this difference from the conventional wisdom with respect to the sign with which forecasts should affect policy, one notes that under the optimal rule it is only forecasts regarding the near future that matter much at all. Even if we consider only the weights put on forecasts for $j \geq 1$ quarters in the future, the mean future horizon of these forecasts, defined by

$$
\sum_{j \geq 1} \alpha_{z, j} j / \sum_{j \geq 1} \alpha_{z, j}
$$

is equal to only 2.2 quarters in the case of our calibrated example with $\gamma=1$. Thus forecasts other than for the first year following the current quarter matter little under the optimal policy. Even more notably, none of the projections beyond the current quarter should receive too great a weight; in our example, the sum of the relative weights on all future quarters,

$$
\sum_{j>0}\left|\alpha_{z, j}\right| / \alpha_{z, 0}
$$

is equal to only 0.39 even in the extreme case $\gamma=1$, while this fraction falls to zero for $1 / \alpha_{z, 0}$. 
small $\gamma$. Thus while a robustly optimal direct instrument rule does have to be forecast-based in the presence of inflation inertia, the degree to which forecasts matter under the optimal policy rule is still relatively small. Instead, a strong response to projections of inflation and the output gap for the current period, as called for by the Taylor rule, continues to be the crucial element of optimal policy.

\subsection{A Robustly Optimal Targeting Rule}

The presence of two roots outside the unit circle in the factorization (2.9) suggests that we ought to solve both of them forward, rather than only one. (This would eliminate the ambiguity about which one to solve forward, that required us to choose between representations (2.11) and (2.12) of optimal policy. Solving both roots forward, we obtain instead a relation of the form

$$
\left(1-\lambda_{1} L\right) \hat{\imath}_{t-1}=-\left(\beta \gamma \lambda_{2} \lambda_{3}\right)^{-1} \mathrm{E}_{t}\left[\left(1-\lambda_{2}^{-1} L^{-1}\right)^{-1}\left(1-\lambda_{3}^{-1} L^{-1}\right)^{-1} \hat{f}_{t}\right]
$$

Once again, this relation can be shown to be equivalent to the Euler equation (2.5).

Proposition 11. Under the assumptions of Proposition 8, a pair of bounded processes

$\left\{\hat{\imath}_{t}, \hat{f}_{t}\right\}$ satisfy (2.15) at all dates $t \geq t_{0}$ if and only if they satisfy (2.10) at all of those same dates.

(See proof in the Appendix.) And an advantage of solving both roots forward is that this relation, unlike either (2.11) or (2.12), has real coefficients for all leads and lags, whether or not there are complex roots.

However, this relation differs from any of the relations $(2.11)-(2.13)$ in that it does not involve $\hat{\imath}_{t}$. Thus it represents a pure targeting rule, one that cannot be interpreted as an instrument rule. However, it follows from Proposition 8 that a commitment to adjust the short-term nominal interest rate so as to achieve a situation in which the bank's projections satisfy (2.15) represents a well-defined policy rule, and one that is furthermore equivalent to any of the three instrument rules discussed in the previous section (if these are defined). 
Hence this provides a relatively simple example of a robustly optimal pure targeting rule, for the case of an economy with inflation inertia.

The optimal target criterion (2.15) can be written in the form

$$
F_{t}(\pi)+\frac{\phi_{x}}{4} F_{t}(x)=\theta_{\pi} \pi_{t-1}+\frac{\theta_{x}}{4} x_{t-1}-\theta_{i}\left(i_{t-1}-i^{*}\right)-\theta_{\Delta} \Delta i_{t-1},
$$

generalizing (1.25). Note that the only difference as to the general form of this targeting rule is the presence of the term indicating dependence of the modified inflation-forecast target on lagged inflation $\pi_{t-1}$. One can also establish the following properties of the optimal coefficients, generalizing our previous results.

Proposition 12. Under the assumptions of Proposition 10, the optimal targeting criterion has a representation (2.16) in which

$$
\begin{gathered}
\phi_{x}=\theta_{x}>0, \\
0<\theta_{\pi} \leq 1,
\end{gathered}
$$

and

$$
\theta_{i}, \quad \theta_{\Delta}>0 .
$$

Furthermore, for fixed values of the other parameters, as $\gamma \rightarrow 0, \theta_{\pi}$ approaches zero and the other parameters approach the non-zero values associated with the target criterion (1.25). Instead, as $\gamma \rightarrow 1, \theta_{\pi}$ approaches 1 .

(Again, the proof is in the Appendix.)

Once again, the optimal inflation-forecast target must be modified in response to variations in the output-gap projection; a higher inflation-forecast target is appropriate if the output gap is projected to fall relative to its recent past level. And once again the optimal inflation-forecast target must be history-dependent, not only because the output-gap modification just mentioned depends upon the relation between the output-gap projections for 

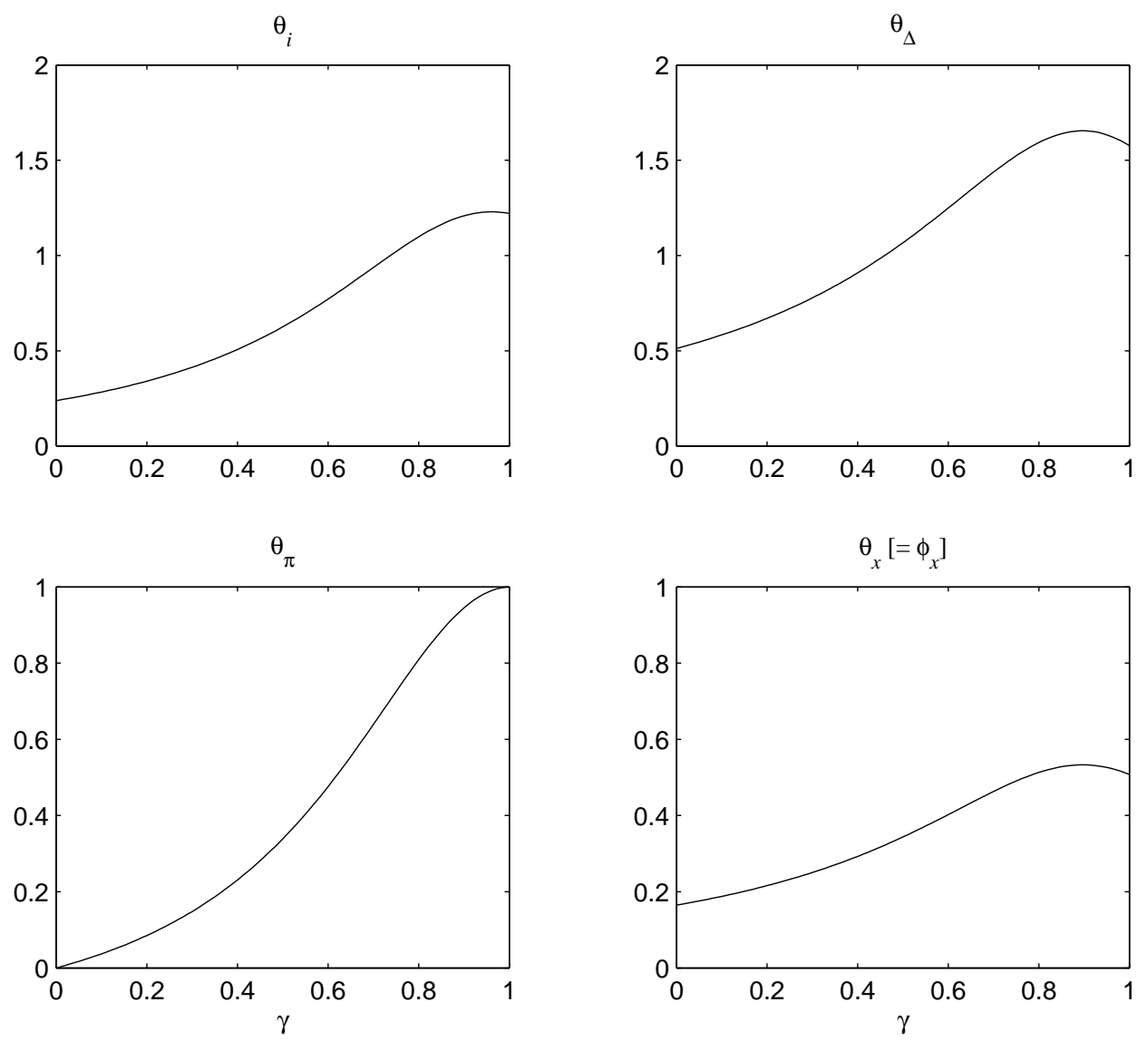

Figure 3: Coefficients of the optimal targeting rule (2.16) as functions of $\gamma$.

current and future periods relative to a past level, but because the appropriate inflationforecast target is lower if nominal interest rates have been high and/or increasing in the recent past.

The additional presence of a coefficient $\theta_{\pi}>0$ when $\gamma>0$ indicates that the modified inflation-forecast target should be higher when recent inflation has been higher; this makes sense given that the distortions associated with inflation variations are greater the greater the departure of the current inflation rate from the rate of automatic price adjustment in response to lagged inflation. In the extreme case $\gamma=1$, the inflation-forecast target is adjusted one hundred percent in response to variations in the recent rate of inflation, since in this case it is actually the rate of change in inflation that one wants to stabilize.

Each of the coefficients in (2.16) is plotted as a function of $\gamma$ in Figure 3, assuming the 

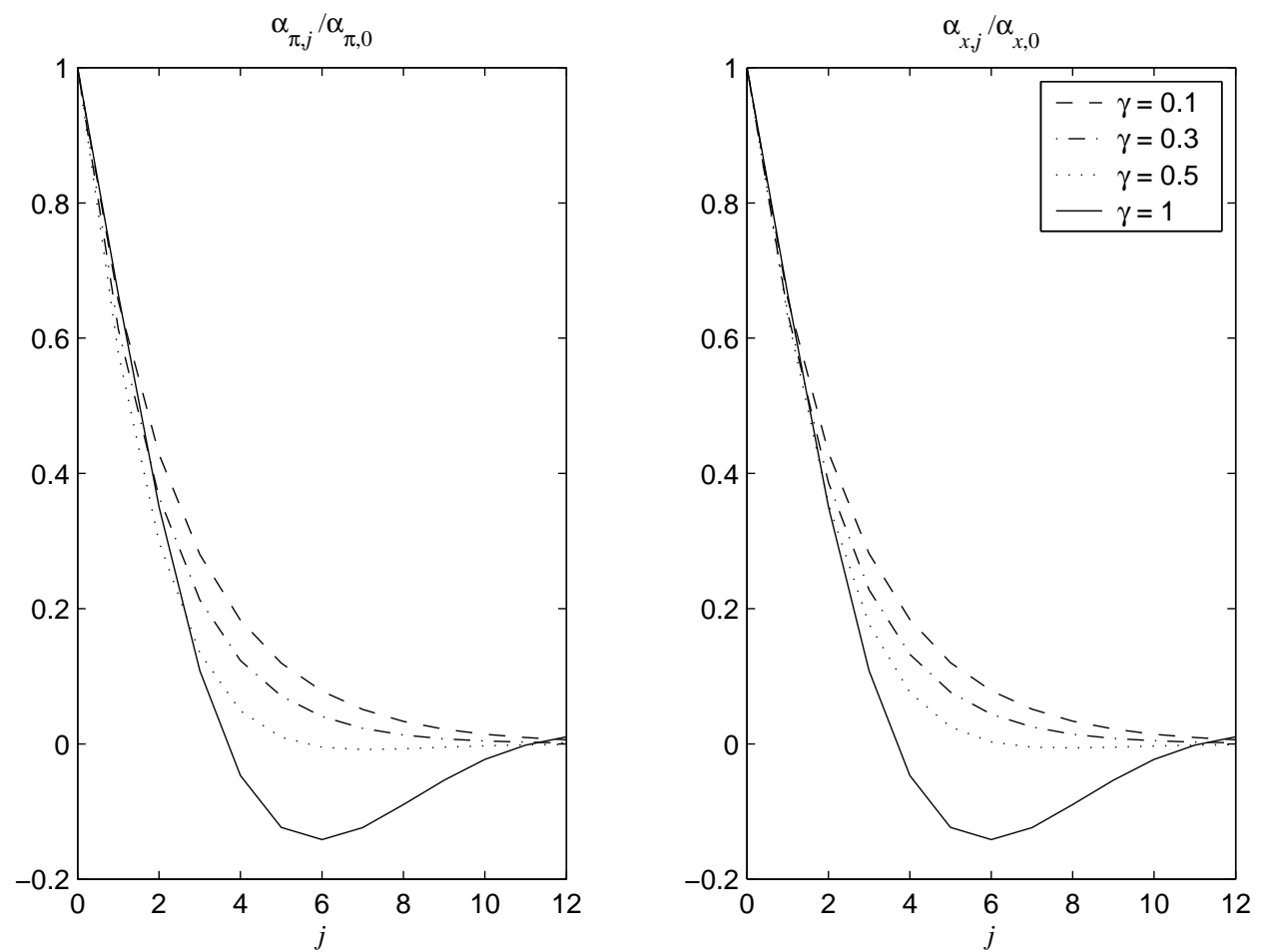

Figure 4: Relative weights on forecasts at different horizons in the optimal rule (2.16).

same calibrated values for the other parameters as before. (There are this time no separate plots for $\theta_{x}$ and $\phi_{x}$, as these coefficients are necessarily identical.) An interesting feature of these results is that each of the coefficients indicating history-dependence $\left(\theta_{\pi}, \theta_{x}, \theta_{i}\right.$, and $\left.\theta_{\Delta}\right)$ increases with $\gamma$. Thus if there is substantial inflation inertia, it is even more important for the inflation-forecast target to vary with changes in recent economic conditions. It is also worth noting that the degree to which the inflation-forecast target should be modified in response to changes in the output-gap projection (indicated by the coefficient $\phi_{x}$ ) increases with $\gamma$. While our conclusion for the baseline model (an optimal targeting rule with $\phi_{x}=.15$ ) might have suggested that this sort of modification of the inflation-forecast target is not too important, we find that a substantially larger response is justified if $\gamma$ is large (the optimal $\phi_{x}$ approaches the value 0.51 for $\gamma=1$ ).

The panels of Figure 4 correspondingly show the relative weights $\alpha_{z, j} / \alpha_{z, 0}$ on the forecasts at different horizons in the optimal target criterion (2.16), for each of several alternative 
values of $\gamma$. As before, this representation of optimal policy is more forward-looking than is the optimal instrument rule; we now find, at least for high enough values of $\gamma$, that the optimal target criterion places non-negligible weight on forecasts more than a year in the future. But it is not necessarily true that a greater degree of inflation inertia justifies a target criterion that with a longer forecast horizon. Increases in $\gamma$ increase the optimal weights on the current-quarter projections of both inflation and the output gap (normalizing the weights to sum to one), and instead make the weights on the projections for quarters more than two quarters in the future less positive. At least for low values of $\gamma$ (in which case the weights are all non-negative), this makes the optimal target criterion less forward-looking. For high values of $\gamma$, increases in $\gamma$ do increase the absolute value of the weights on forecasts for dates one to two years in the future (these become more negative). But even in this case, the existence of inflation inertia does not justify the kind of response to longer-horizon forecasts that is typical of inflation-forecast targeting central banks. An increase in the forecast level of inflation and/or the output gap during the second year of a bank's current projection should justify a loosening of current policy, in the sense of a policy intended to raise projected inflation and/or the output gap in the next few quarters.

The considerations that might lead one to choose the pure targeting rule as opposed to the optimal instrument rule as a policy commitment remain as in our earlier discussion. We note once again that the targeting rule (2.16) has the advantage of remaining well-defined in the limit of a stabilization objective with $\lambda_{i}=0$. In this limit, (2.15) reduces to the form

$$
\mathrm{E}_{t}\left[\left(1-\lambda_{2}^{-1} L^{-1}\right)^{-1}\left(1-\lambda_{3}^{-1} L^{-1}\right)^{-1}\left(1-\beta \gamma L^{-1}\right) \hat{q}_{t}\right]=0
$$

which corresponds once again to a target criterion of the form $(2.16)$, but with $\theta_{i}=\theta_{\Delta}=0$. This relation, however, is satisfied if and only if $\tilde{q}_{t}=0,{ }^{36}$ and so a simpler expression for the

\footnotetext{
${ }^{36}$ Here it is worth noting that the only reason that we were not similarly able to reduce (2.15) to an equivalent target criterion that involves no dependence upon forecasts was the presence of the non-zero terms involving $\hat{\imath}_{t-1}$ and $\hat{\imath}_{t-2}$. Thus there is an important connection between the irreducible forecast-dependence of the optimal target criterion (2.16) and the need for a more complex kind of history-dependence of optimal policy in the case that $\lambda_{i}>0$.
} 
optimal targeting rule in this limit is simply

$$
\pi_{t}+\frac{\lambda_{x}}{\kappa} x_{t}=\gamma \pi_{t-1}+\frac{\lambda_{x}}{\kappa} x_{t-1}
$$

In this simple case, we observe that the optimal targeting rule need not be forward-looking at all, regardless of the degree of inflation inertia.

In the special case that $\gamma=1,(2.17)$ can be further simplified: an equivalent targeting rule is simply

$$
\pi_{t}+\frac{\lambda_{x}}{\kappa} x_{t}=\bar{\pi}
$$

where $\bar{\pi}$ is an arbitrary constant. ${ }^{37}$ In this case, the target for the current quarter's inflation projection must be modified in response to variations in the current output-gap projection, but neither lagged values nor projections farther in the future need be taken account of. This might seem to contradict our earlier remarks about the necessary history-dependence of optimal policy. However, one should note that in the case that $\lambda_{i}=0$ and $\gamma=1$, the only target variables are $\Delta \pi_{t}$ and $x_{t}$ (not $\left.\pi_{t} !\right)$, and the only constraint upon the feasible evolution of these variables is provided by the aggregate-supply relation (2.1), which is purely forwardlooking in terms of these variables. The only state variables that matter for defining the feasible paths for the target variables from date $t$ onward are the exogenous states needed to identify $u_{t}$ and the conditional expectations $\mathrm{E}_{t} u_{t+j}$ for all future horizons. Thus, in terms of our previous definition, a "purely forward-looking" target criterion would be one that refers only to projections for $\Delta \pi_{t}$ and $x_{t}$ in current and future quarters, and possibly to the exogenous states just mentioned. The target criterion (2.18) is not purely forward-looking in this sense; for it makes the target for current inflation acceleration $\Delta \pi_{t}$ dependent upon past inflation, even though that variable is irrelevant to both the policymaker's loss function and to the constraints upon the future evolution of the relevant target variables. ${ }^{38}$

\footnotetext{
${ }^{37}$ If the central bank commits to enforcement of the target criterion (2.17) from some date $t_{0}$ onward, then the constant is determined by the initial conditions at the time that the rule is adopted: $\bar{\pi}=\pi_{t_{0}-1}+$ $\left(\lambda_{x} / \kappa\right) x_{t_{0}-1}$.

${ }^{38}$ In supposing that it is possible for the absolute level of the inflation rate to be irrelevant for the possible future evolution of the rate of change of inflation, we are obviously ignoring the consequences of the zero lower bound on nominal interest rates. However, the choice of a loss function with $\lambda_{i}=0$ is only appropriate
} 


\section{$3 \quad$ Further Extensions}

Here we consider, more briefly, some extensions of the method introduced above to more complex (but more realistic) settings. As we shall see, not only can similar methods be employed in a broader range of cases, but certain important conclusions obtained in the above examples regarding the character of an optimal policy rule continue to apply in more general settings.

\subsection{Sticky Wages and Prices}

As discussed in Woodford (2002, chap. 3), a more realistic model will allow for sticky wages as well as prices. Here we consider how the form of an optimal policy rule changes in the case that wages and prices are both sticky to a similar extent. We assume a structural model with monopolistic competition among the suppliers of differentiated types of labor and Calvo-style staggering of wage adjustment, as in Woodford (2002, chap. 3, sec. 4.1), and utility-based stabilization objectives in accordance with the derivation in Woodford (2002, chap. 6). For the sake of brevity, we proceed directly to the case of a model in which both wages and prices are partially indexed to lagged inflation; the model of Erceg et al. (2000), in which there is no such indexation, can be treated as a limiting case of this model.

As in Woodford (2002, chap. 3), the structural equations of our model are ${ }^{39}$

$$
\begin{gathered}
\pi_{t}-\gamma_{p} \pi_{t-1}=\kappa_{p}\left(x_{t}+u_{t}\right)+\xi_{p}\left(\hat{w}_{t}-\hat{w}_{t}^{n}\right)+\beta \mathrm{E}_{t}\left[\pi_{t+1}-\gamma_{p} \pi_{t}\right], \\
\pi_{t}^{w}-\gamma_{w} \pi_{t-1}=\kappa_{w}\left(x_{t}+u_{t}\right)+\xi_{w}\left(\hat{w}_{t}^{n}-\hat{w}_{t}\right)+\beta \mathrm{E}_{t}\left[\pi_{t+1}^{w}-\gamma_{w} \pi_{t}\right],
\end{gathered}
$$

together with the intertemporal IS relation (1.1). Here $\pi_{t}^{w}$ represents nominal wage inflation, $\hat{w}_{t}$ is the deviation of the log real wage from its steady-state level, $\hat{w}_{t}^{n}$ represents the $\log$ deviation of the "natural real wage" - i.e., the equilibrium real wage in the case of complete wage and price flexibility — from its stead-state level, and the coefficients $\xi_{p}, \xi_{w}, \kappa_{p}, \kappa_{w}$ are

if one judges that in practice this constraint does not bind, so that the optimal policy problem can be considered as if there were no such constraint.

${ }^{39}$ Here we have rewritten $\log Y_{t}-\log Y_{t}^{n}$ as $x_{t}+u_{t}$, where as in our basic model, $x_{t}$ is the gap between actual and efficient output, and $u_{t}$ represents inefficient variation in the natural rate of output. 
all positive. The coefficients $0 \leq \gamma_{p}, \gamma_{w} \leq 1$ indicate the degree of indexation of prices and wages respectively to the lagged price index, analogous to the indexation of prices in the model of section 2. (The model of Erceg et al. corresponds to the special case in which $\left.\gamma_{w}=\gamma_{p}=0.\right)$

Under the microeconomic foundations for these relations discussed in Woodford (2002, chap. 3), the appropriate welfare-theoretic stabilization objective is a discounted criterion of the form (1.3), with a period loss function of the form ${ }^{40}$

$$
L_{t}=\lambda_{p}\left(\pi_{t}-\gamma_{p} \pi_{t-1}\right)^{2}+\lambda_{w}\left(\pi_{w t}-\gamma_{w} \pi_{t-1}\right)^{2}+\lambda_{x}\left(x_{t}-x^{*}\right)^{2}+\lambda_{i}\left(i_{t}-i^{*}\right)^{2}
$$

We wish to consider policies that minimize the criterion defined by (1.3) and (3.3), subject to the constraints imposed by the structural equations (1.1) and (3.1) - (3.2).

Using the same Lagrangian method as before to characterize optimal policy, we obtain a set of first-order conditions

$$
\begin{gathered}
\lambda_{p}\left[\left(\pi_{t}-\gamma_{p} \pi_{t-1}\right)-\beta \gamma_{p} \mathrm{E}_{t}\left(\pi_{t+1}-\gamma_{p} \pi_{t}\right)\right]-\lambda_{w} \beta \gamma_{w} \mathrm{E}_{t}\left(\pi_{w, t+1}-\gamma_{w} \pi_{t}\right)+\beta^{-1} \sigma \Xi_{1, t-1} \\
+\beta \gamma_{p} \mathrm{E}_{t}\left(\Xi_{2, t+1}-\Xi_{2 t}\right)-\left(\Xi_{2 t}-\Xi_{2, t-1}\right)+\beta \gamma_{w} \mathrm{E}_{t}\left(\Xi_{3, t+1}-\Xi_{3 t}\right)-\Xi_{4 t}=0, \\
\lambda_{w}\left(\pi_{w t}-\gamma_{w} \pi_{t-1}\right)-\left(\Xi_{3 t}-\Xi_{3, t-1}\right)+\Xi_{4 t}=0, \\
\lambda_{x}\left(x_{t}-x^{*}\right)-\Xi_{1 t}+\beta^{-1} \Xi_{1, t-1}+\kappa_{p} \Xi_{2 t}+\kappa_{w} \Xi_{3 t}=0, \\
\lambda_{i}\left(i_{t}-i^{*}\right)-\sigma \Xi_{1 t}=0, \\
\xi_{p} \Xi_{2 t}-\xi_{w} \Xi_{3 t}-\Xi_{4 t}+\beta \mathrm{E}_{t} \Xi_{4, t+1}=0,
\end{gathered}
$$

where $\Xi_{1 t}, \Xi_{2 t}, \Xi_{3 t}$ are the Lagrange multipliers associated with constraints (1.1), (3.1) and (3.2) respectively, and $\Xi_{4 t}$ is the multiplier associated with the constraint

$$
\hat{w}_{t}=\hat{w}_{t-1}+\pi_{w t}-\pi_{t} .
$$

\footnotetext{
${ }^{40}$ The welfare-theoretic loss function for this model when $\gamma_{w}=\gamma_{p}=0$ and there is no penalty for interestrate variability is derived in Woodford (2001b, chap. 6, sec. 4.4). When $\gamma_{w}, \gamma_{p}>0$, the relation of wage and price dispersion to wage and price inflation changes in the way discussed in Woodford (2002, chap. 6, sec. 2.2 ), resulting in the modification indicated here of the first two terms of the loss function. The justification for the final term is the same as in the flexible-wage model above.
} 
A case in which these equations are especially easy to interpret is the special case discussed earlier, in which $\kappa_{w}=\kappa_{p}=\kappa$ (so that wages and prices are sticky to a similar degree) and $\gamma_{w}=\gamma_{p}=\gamma$ (so that wages and prices are indexed to lagged inflation to the same degree). In this case, we can add (3.4) to (3.5), use (3.6) to substitute for $\Xi_{2 t}+\Xi_{3 t}$ and (3.7) to substitute for $\Xi_{1 t}$, and finally obtain

$$
\begin{aligned}
& \left(\lambda_{p} \pi_{t}+\lambda_{w} \pi_{w t}-\gamma \pi_{t-1}\right)-\beta \gamma \mathrm{E}_{t}\left(\lambda_{p} \pi_{t+1}+\lambda_{w} \pi_{w, t+1}-\gamma \pi_{t}\right)=\frac{\lambda_{i}}{\kappa \sigma}\left(\Delta i_{t}-\beta^{-1} \Delta i_{t-1}\right) \\
& -\beta \gamma \mathrm{E}_{t}\left[\frac{\lambda_{i}}{\kappa \sigma}\left(\Delta i_{t+1}-\beta^{-1} \Delta i_{t}\right)\right]-\frac{\lambda_{i}}{\beta}\left(i_{t-1}-i^{*}\right)-\frac{\lambda_{x}}{\kappa}\left(\Delta x_{t}-\beta \gamma \mathrm{E}_{t} \Delta x_{t+1}\right) .
\end{aligned}
$$

This is again an Euler equation of the form (2.5), where again $A(L)$ is defined by (2.6) and $f_{t}$ is defined by $(2.7)-(2.8)$; the only difference is that is in the last of these equations, $\pi_{t}^{q d}$ is now defined as

$$
\pi_{t}^{q d} \equiv \lambda_{p} \pi_{t}+\lambda_{w} \pi_{w t}-\gamma \pi_{t-1}
$$

rather than as in (2.4). It follows that optimal policy rules are of essentially the same form as for the model with only sticky prices, except that terms that previously involved only price inflation will now involve both wage and price inflation.

In the case that $\gamma=0$ (the model of Erceg et al., 2000), we obtain an especially simple result. The optimal instrument rule is again of the form (1.17), except that instead of responding to current and lagged price inflation $\pi_{t}$, the rule prescribes a response (with the same coefficients as before) to a weighted average of wage and price inflation,

$$
\bar{\pi}_{t} \equiv \lambda_{p} \pi_{t}+\lambda_{w} \pi_{w t}
$$

For the calibrated parameter values suggested in Table 2 below, this index involves equal weights on wage and price inflation. Similarly, the optimal pure targeting rule is again of the form $(1.25)$, except that the inflation projection $F_{t}(\pi)$ is replaced by a projection $F_{t}(\bar{\pi})$ of the average of wage and price inflation just defined, with the same weights on various future horizons as before. Once again, wage inflation should receive a similar weight as price inflation in the inflation forecast that is used in the central bank's forecast-targeting procedure. 
It is worth noting that we obtain different coefficients here for the optimal policy rule than in section 2 only because the welfare-theoretic loss function is different in the case that wages as well as prices are sticky. If instead of (3.3) we were to assume a loss function of the form (1.4) with arbitrary weights - a common assumption in non-welfare-theoretic analyses of monetary policy rules — we would again have obtained precisely the same optimal policy rules as in section 2. (This can be seen from the fact that (3.9) reduces to $(2.4)$ if $\lambda_{w}=0$.) Thus sticky wages need not imply any difference in the nature of the tradeoff between inflation and output-gap stabilization available to the central bank; the main significance of wage stickiness is that it makes wage stabilization an appropriate objective for policy, with consequences for the form of inflation index that belongs in an optimal policy rule.

In the case of indexation to lagged inflation, the roots of the lag polynomial $A(L)$ are the same as in the previous section, yielding the same forms as before for alternative optimal policy rules. (The three optimal instrument rules are each defined for the same values of $\gamma$ as above; the unique optimal pure targeting rule is again defined for all $\gamma$.) Each of the three optimal instrument rules can be written in the form

$$
i_{t}=\left(1-\rho_{1}\right) i^{*}+\rho_{1} i_{t-1}+\rho_{2} \Delta i_{t-1}+\phi_{p} F_{t}(\pi)+\phi_{w} F_{t}\left(\pi_{w}\right)+\frac{\phi_{x}}{4} F_{t}(x)-\theta_{\pi} \pi_{t-1}-\frac{\theta_{x}}{4} x_{t-1},
$$

where the coefficients $\rho_{1}, \rho_{2}, \phi_{x}, \theta_{\pi}$, and $\theta_{x}$ and the coefficients $\left\{\alpha_{j}^{x}\right\}$ are all the same functions of the model parameters as in (2.14), for each of the three rules. The coefficients multiplying the price and wage inflation forecasts satisfy

$$
\phi_{p}+\phi_{w}=\phi_{\pi}
$$

and

$$
\phi_{p} \alpha_{j}^{p}+\phi_{w} \alpha_{j}^{w}=\phi_{\pi} \alpha_{j}^{\pi}
$$

for each $j \geq 0$, where $\phi_{\pi}$ and the $\left\{\alpha_{j}^{\pi}\right\}$ are the coefficients multiplying the inflation forecasts in (2.14). Thus if wages and prices are forecasted to increase at the same rate, the effect of these inflation forecasts on the desired interest-rate setting is the same as before. However, if the wage and price inflation forecasts differ, optimal policy now depends on the wage inflation forecast as well. 
Table 2: Parameter values in a calibrated model with sticky wages and prices.

\begin{tabular}{|c|c|}
\hline \multicolumn{2}{|c|}{ Structural parameters } \\
\hline$\alpha_{w}$ & 0.66 \\
$\alpha_{p}$ & 0.62 \\
$\beta$ & 0.99 \\
$\sigma^{-1}$ & 0.16 \\
$\omega_{w}$ & 0.28 \\
$\omega_{p}$ & 0.43 \\
$\theta_{w} \phi^{-1}, \theta_{p}$ & 7.88 \\
$\xi_{w}, \xi_{p}$ & .055 \\
$\kappa_{w}, \kappa_{p}$ & .024 \\
\hline \hline \multicolumn{2}{|c|}{ Loss function } \\
\hline$\lambda_{w}, \lambda_{p}$ & 0.50 \\
$\lambda_{x}$ & .048 \\
$\lambda_{i}$ & .236 \\
\hline
\end{tabular}

When $\gamma>0$, the optimal rule no longer involves only projections of a single weighted average of wage and price inflation; this is because both wages and prices are (by assumption) indexed only to lagged price inflation, and not to lagged wage inflation. The difference in the optimal responses to wage inflation as opposed to price inflation is illustrated in Figures 5 and 6 for a calibrated example, with parameter values displayed in Table 2 . Here we assume that $\xi_{w}=\xi_{p}, \kappa_{w}=\kappa_{p}$, and $\theta_{w} \phi^{-1}=\theta_{p}$. The values assumed for $\beta, \sigma, \theta_{p}$, and $\kappa_{p}$ are taken from the estimates of the IS equation and price inflation equation by Rotemberg and Woodford (1997); $;^{41}$ the value of $\xi_{p}$ is instead taken from the inflation equation estimated by Sbordone (2002a), which relates inflation to real marginal cost rather than to the output gap. ${ }^{42}$ The values of $\alpha_{w}, \alpha_{p}, \omega_{w}$ and $\omega_{p}$ implied by these estimates are also shown in the table, ${ }^{43}$ as are the implied coefficients $\lambda_{w}, \lambda_{p}$ and $\lambda_{x}$ of the welfare-theoretic loss function. Note that wage

\footnotetext{
${ }^{41}$ Note that if $\kappa_{w}=\kappa_{p}$, as assumed here, then the real wage is unaffected by monetary policy, as discussed in Woodford (2002, chap. 3, sec. 4.2). In this case the Rotemberg-Woodford inflation equation is correctly specified even when wages are sticky (though their welfare analysis would not be correct), and their parameter " $\kappa$ " corresponds to $\kappa_{p}$ here.

${ }^{42}$ Note that Sbordone's inflation equation is equally valid regardless of whether wages are sticky or not.

${ }^{43}$ Even though our parameter values have been taken from two different studies using different data sets, the implied values of these parameters are reasonable, and not too different from the estimates of Amato and Laubach (2001a); see, for example, Table 4.2 of Woodford (2002).
} 

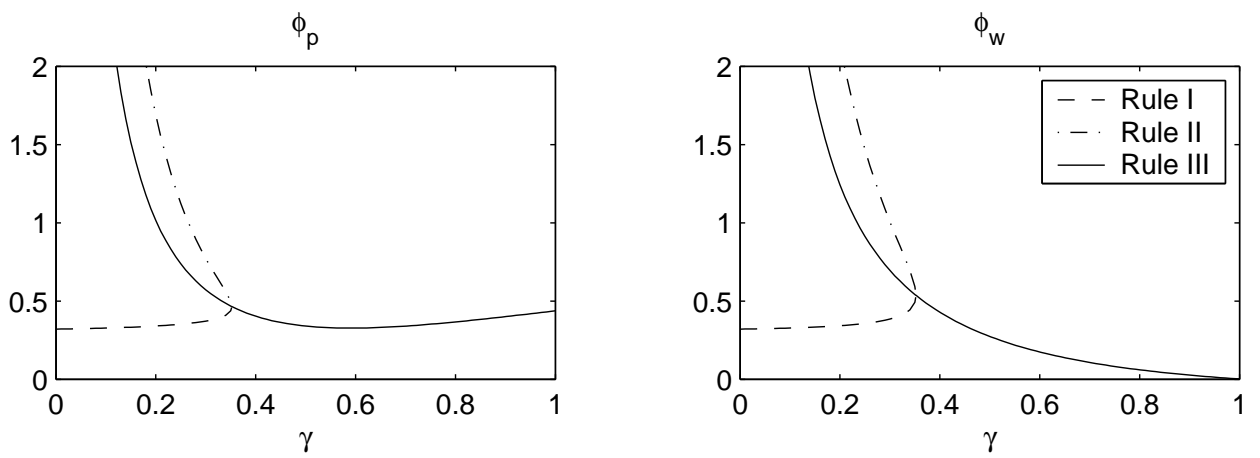

Figure 5: Coefficients of the optimal targeting rule (3.10) as functions of $\gamma$. Coefficients not shown are the same as in Figure 1.

inflation and price inflation receive equal weight, and that the relative weight on outputgap stabilization is the same as in Table 1 . We also assume the same relative weight $\lambda_{i}$ on interest-rate stabilization as in the calibration above of the flexible-wage model. ${ }^{44}$

Figure 5 shows the value of the coefficients $\phi_{p}$ and $\phi_{w}$ in the optimal instrument rule (3.10), for alternative values of $\gamma$ ranging between zero and one. (Again the values are shown for each of rules I, II and III in the cases where these exist; the minimally inertial rule corresponds to rule I for low values of $\gamma$, and rule III for high values.) Similarly, Figure 6 shows the relative weights on the inflation and output-gap forecasts at different horizons in the optimal rule.

We note that for moderate positive values of $\gamma$, it continues to be the case that the coefficients on the price-inflation forecasts are quite similar to those on the wage-inflation forecasts; essentially, the coefficient $\phi_{\pi}$ shown in Figure 1 is split roughly equally between the coefficients $\phi_{p}$ and $\phi_{w}$, while the relative weights on forecasts at different horizons remain similar to those shown in Figure 2. Thus it is not too bad an approximation to optimal policy to choose the same rule as the one described in section 2 , but to respond to forecasts

\footnotetext{
${ }^{44}$ If $\lambda_{i}$ resulted solely from the existence of transactions frictions, as discussed in Woodford (2002, chap. 6, sec. 4.1), the same calibrated value would be appropriate regardless of the assumed degree of wage stickiness. In the case that $\lambda_{i}$ is chosen to reflect the advantages of lower interest-rate variability as a result of the zero bound, as in Woodford (1999a), then the appropriate value would depend on the assumed variance of disturbances. In this case, the appropriate value is not independent of whether we assume wages to be sticky, because the other stabilization objectives are not the same in this case; but we do not here consider the degree to which the appropriate value of $\lambda_{i}$ should change.
} 

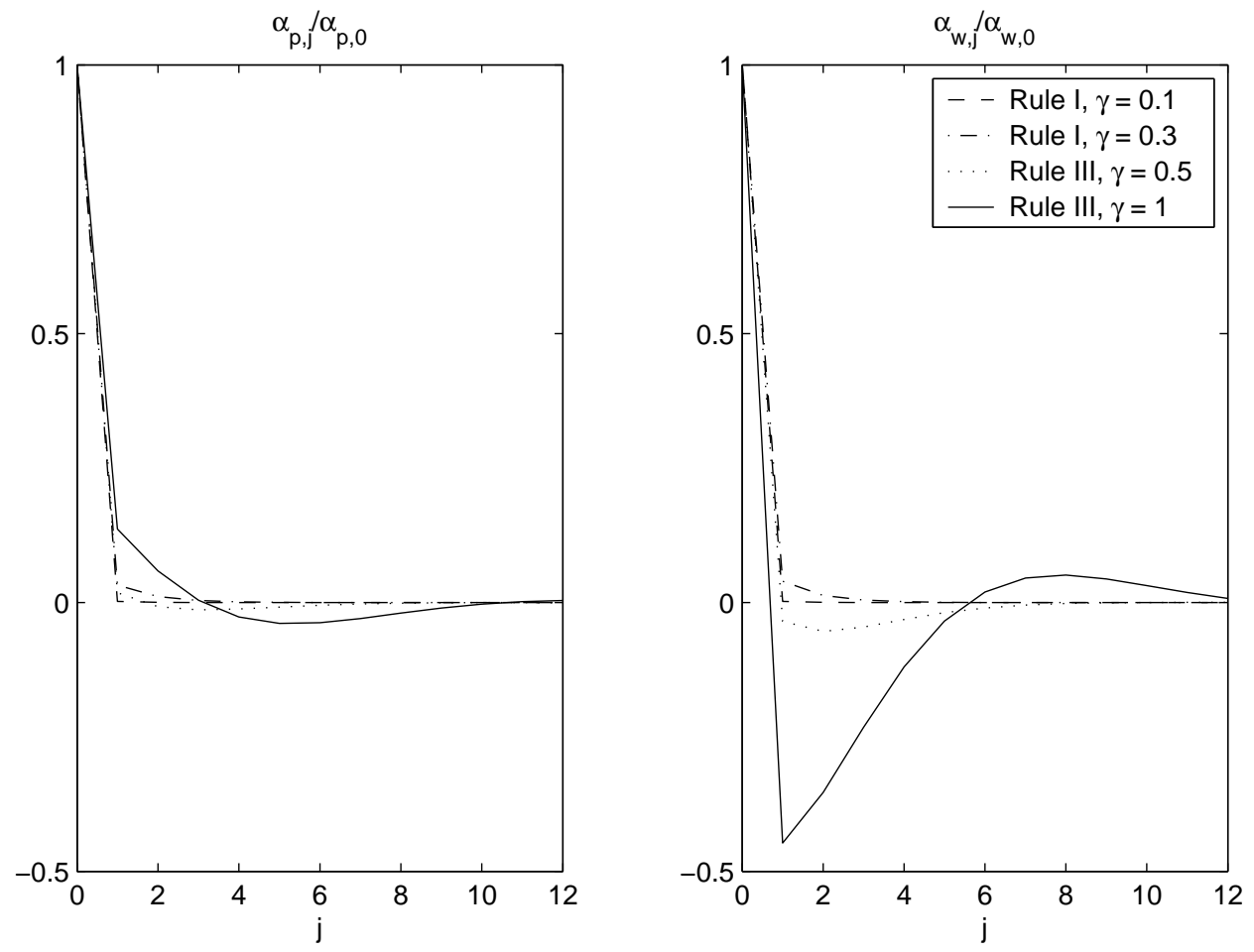

Figure 6: Relative weights on forecasts at different horizons in the optimal rule (3.10).

of an index that is a (roughly equally-weighted) average of wage and price inflation. For larger values of $\gamma$, however, the optimal responses to forecasts of wage and price inflation are substantially different. The optimal value of $\phi_{p}$ remains positive, and similar in magnitude to the previous coefficient $\phi_{\pi}$, while the optimal value of $\phi_{w}$ falls to zero as $\gamma$ approaches one. This does not mean that it ceases to be optimal to respond to forecasts of wage inflation, only that the sum of the weights at different horizons is zero; ${ }^{45}$ that is, when $\gamma=1$ the rule prescribes a response only to the forecasted rate of acceleration of wage inflation, rather than the rate of wage inflation itself (given the expected rate of price inflation). Specifically, the optimal rule prescribes a negative response to expected deceleration of wage inflation over the next three quarters relative to the current rate of wage inflation; it also prescribes

\footnotetext{
${ }^{45}$ This explains why we plot relative weights rather than the weights $\alpha_{w, j}$ in Figure 6 . If we normalize the $\alpha_{w, j}$ to sum to one, then the weights are undefined in the limiting case $\gamma=1$. Nonetheless, the relative weights have well-defined limiting values, shown in the figure. The coefficients multiplying any given forecast of wage inflation - i.e., the products $\phi_{w} \alpha_{w, j}$ - also remain well-defined, so there is a well-defined optimal policy rule in this case.
} 
a (weaker) positive response to expected acceleration of wage inflation farther in the future.

Despite these complications, we note that it continues to be the case that optimal policy depends very little on inflation forecasts (either for wages or prices) farther in the future than the coming year, even in the case that there is substantial inflation inertia in both wages and prices. And even with regard to forecasts for the coming year, current interest rates should respond most strongly (and in particular, most positively) to projected wage and price inflation in the current quarter, rather than to forecasted inflation later in the year. Thus there is once again little support for the kinds of forward-looking rules sometimes offered as descriptions of the behavior of current inflation-targeting central banks.

\subsection{Delays in the Effects of Monetary Policy}

Empirical models such as those of Rotemberg and Woodford (1997), Amato and Laubach (2001a), Christiano et al. (2001), Altig et al. (2002), or Boivin and Giannoni (2002) differ from the simple models discussed above in that both output and inflation are predetermined, so that neither is immediately affected by an unexpected change in policy. Here we consider the consequences for optimal policy of allowing for such delays in the effect of policy, modeled in the way described in Woodford (2002, chap. 4, sec. 4).

Let us consider a model with flexible wages, but sticky prices indexed to lagged inflation, as in section 2, but now assuming that both price changes and aggregate private demand are predetermined $d$ periods in advance, for some $d \geq 0$. For simplicity, let us suppose that the efficient level of output is also known $d$ periods in advance, so that the output gap is also a predetermined variable. ${ }^{46}$ In this case, the structural equations of our model are

$$
\begin{gathered}
x_{t}=\mathrm{E}_{t-d} x_{t+1}-\sigma \mathrm{E}_{t-d}\left(i_{t}-\pi_{t+1}-r_{t}^{n}\right), \\
\pi_{t}-\gamma \pi_{t-1}=\kappa \mathrm{E}_{t-d} x_{t}+\beta \mathrm{E}_{t-d}\left(\pi_{t+1}-\gamma \pi_{t}\right)+\mathrm{E}_{t-d} u_{t} .
\end{gathered}
$$

\footnotetext{
${ }^{46}$ Alternatively, in equations $(3.11)-(3.12)$ we may interpret $x_{t}$ to mean $\hat{Y}_{t}-\mathrm{E}_{t-d} \hat{Y}_{t}^{e}$. In this case, the loss function $(1.3)-(2.2)$ is still correct, up to terms (involving the component of $\hat{Y}_{t}^{e}$ that is not forecastable $d$ periods in advance) that are independent of policy.
} 
The welfare-theoretic loss function continues to be given by (1.3) and (2.2). The Lagrangian associated with our policy problem is then of the form

$$
\begin{aligned}
\mathcal{L}= & \mathrm{E}_{0} \sum_{t=0}^{\infty} \beta^{t}\left\{\frac{1}{2}\left(\pi_{t}^{q d}\right)^{2}+\frac{\lambda_{x}}{2}\left(x_{t}-x^{*}\right)^{2}+\frac{\lambda_{i}}{2}\left(i_{t}-i^{*}\right)^{2}+\right. \\
& \left.\Xi_{1, t-d}\left[x_{t}-\mathrm{E}_{t-d} x_{t+1}+\sigma \mathrm{E}_{t-d}\left(i_{t}-\pi_{t+1}-r_{t}^{n}\right)\right]+\Xi_{2, t-d}\left[\pi_{t}^{q d}-\kappa \mathrm{E}_{t-d} \pi_{t+1}^{q d}-\mathrm{E}_{t-d} u_{t}\right]\right\},
\end{aligned}
$$

where $\pi_{t}^{q d}$ again denotes the quasi-differenced inflation rate (2.4). Here we write $\Xi_{1, t-d}, \Xi_{2, t-d}$ for the multipliers associated with constraints (3.11) and (3.12) respectively, to indicate that each multiplier is determined at date $t-d$, given that there is one such constraint for each possible state of the world at date $t-d$.

Using the law of iterated expectations, the Lagrangian can equivalently be written as

$$
\begin{aligned}
\mathcal{L}=\mathrm{E}_{0} \sum_{t=d}^{\infty} \beta^{t}\left\{\frac{1}{2}\left(\pi_{t}^{q d}\right)^{2}+\frac{\lambda_{x}}{2}\left(x_{t}-x^{*}\right)^{2}+\frac{\lambda_{i}}{2}\left(i_{t}-i^{*}\right)^{2}+\right. & \\
\Xi_{1, t-d}\left[x_{t}-x_{t+1}+\sigma\left(i_{t}-\right.\right. & \left.\left.\left.\pi_{t+1}\right)\right]+\Xi_{2, t-d}\left[\pi_{t}^{q d}-\kappa \pi_{t+1}^{q d}\right]\right\} \\
& +\frac{\lambda_{i}}{2} \mathrm{E}_{0} \sum_{t=0}^{d-1} \beta^{t}\left(i_{t}-i^{*}\right)^{2}
\end{aligned}
$$

dropping terms that are independent of policy. The first-order conditions that characterize an optimal once-and-for-all commitment as of date zero are given by

$$
\pi_{t}^{q d}-\beta \gamma \mathrm{E}_{t-d} \pi_{t+1}^{q d}-\beta^{-1} \sigma \Xi_{1, t-d-1}-\beta \gamma \mathrm{E}_{t-d} \Xi_{2, t-d+1}+(1+\beta \gamma) \Xi_{2, t-d}-\Xi_{2, t-d-1}=0
$$

together with conditions $(1.14)-(1.15)$, but with $\Xi_{i, t-d}$ substituted for $\Xi_{i t}$ (for $\left.i=1,2\right)$ in the latter equations. Each of the first-order conditions just listed holds for each $t \geq d$. In addition, $\pi_{d-1}$ is given as an initial condition, the initial lagged Lagrange multipliers satisfy (1.16), and one has additional first-order conditions

$$
i_{t}=i^{*}
$$

for the interest rate in periods $t=0, \ldots, d-1$. Note, however, that these last conditions, that relate only to the first few periods following the adoption of the optimal commitment, are irrelevant to the characterization of optimal policy from a timeless perspective. 
As above, we can use conditions $(1.14)-(1.15)$ to substitute for $\Xi_{1, t-d}$ and $\Xi_{2, t-d}$ in (3.14), obtaining an Euler equation of the form

$$
A_{1}(L)\left(i_{t}-i^{*}\right)+\beta \gamma \mathrm{E}_{t-d}\left(i_{t+1}-i^{*}\right)=-\mathrm{E}_{t-d} f_{t}
$$

where $A_{1}(L)$ is the quadratic lag polynomial such that

$$
A(L)=\beta \gamma+L A_{1}(L)
$$

is the polynomial defined in (2.6), and $f_{t}$ is again defined in (2.7) - (2.8). It follows from this that under a policy that is optimal from a timeless perspective, $i_{t}$ depends solely on public information at date $t-d$. Hence in the case of structural equations of this kind, there would be no change in the character of optimal policy were one to impose the constraint that the interest-rate operating target must be chosen in advance, as proposed for example by McCallum and Nelson (1999).

Taking the expectation of (3.15) conditional upon information at date $t-d$, one obtains

$$
\mathrm{E}_{t-d}\left[A(L)\left(i_{t+1}-i^{*}\right)\right]=-\mathrm{E}_{t-d} f_{t}
$$

which is identical to (2.5) except for the conditioning information set. The same manipulations as before can then be used to derive the same form of representations for optimal policy, with the change in the conditioning information set for expectations. There are once again the three possible forms for an optimal instrument rule discussed in section 2 above, and each exists for the same values of $\gamma$ as before. Each of the three rules is of the form

$$
i_{t}=\left(1-\rho_{1}\right) i^{*}+\rho_{1} i_{t-1}+\rho_{2} \Delta i_{t-1}+\phi_{\pi} \mathrm{E}_{t-d} F_{t}(\pi)+\frac{\phi_{x}}{4} \mathrm{E}_{t-d} F_{t}(x)-\theta_{\pi} \pi_{t-1}-\frac{\theta_{x}}{4} x_{t-1}
$$

where the coefficients are exactly the same functions of the model parameters as in (2.14). Similarly, optimal policy can again be represented by a pure targeting rule with a target criterion of the form

$$
\mathrm{E}_{t-d} F_{t}(\pi)+\frac{\phi_{x}}{4} \mathrm{E}_{t-d} F_{t}(x)=\theta_{\pi} \pi_{t-1}+\frac{\theta_{x}}{4} x_{t-1}-\theta_{i}\left(i_{t-1}-i^{*}\right)-\theta_{\Delta} \Delta i_{t-1}
$$


where the coefficients are exactly the same functions of the model parameters as in (2.16). Thus the optimal policy rules are of exactly the same form as before, except that now the period $t$ interest rate should be chosen in period $t-d$, and on the basis of the inflation and output-gap projections that are available at that earlier date. The projections, however, should be for the same time periods as before.

In the case that $\gamma=0$ (our baseline model, with a standard "New Keynesian Phillips curve", except for the $d$-period delays), the projections in (3.16) again are only for inflation and the output gap in period $t$. Since both of these variables are known at date $t-d$, according to our model, the optimal instrument rule is once again of the form (1.17), with coefficients $(1.18)-(1.19)$. Thus in this case there is no change at all in the optimal policy rule. It remains true that the delays imply that it is optimal for nominal interest rates to be perfectly forecastable $d$ periods in advance. However, this principle does not imply that a rule that prescribes a response to contemporaneous inflation and output-gap variations, as under the Taylor rule, is therefore suboptimal. For under our assumptions, inflation and the output gap are themselves completely forecastable $d$ periods in advance. This example shows that an optimal policy rule need not be at all forward-looking, even in the case that the effects of monetary policy are entirely delayed.

Of course, even if there are no effects of a change in monetary policy until $d$ periods later, it need not follow that inflation and the output gap are completely predetermined. Only the components of these variables that are affected by monetary policy need be predetermined. In a more complex model, we may assume that the forecastable components of inflation and the output gap, $\mathrm{E}_{t-d} \pi_{t}$ and $\mathrm{E}_{t-d} x_{t}$, satisfy equations (3.11) - (3.12), while the observed variables are equal to these forecastable components plus exogenous disturbance terms. ${ }^{47}$ In this case, both equations (3.11) - (3.12) should include additional unforecastable disturbance terms, as in the model discussed in Svensson and Woodford (1999). In this case, the Lagrangian

\footnotetext{
${ }^{47}$ An IS relation of this form is presented in Woodford (2002, chap. 4, sec. 4.1), where the additional disturbance results from the unforecastable components of government purchases and/or the efficient level of output. In the case of inflation, one might suppose that wholesale prices are determined $d$ periods in advance, and satisfy (3.12), while the retail price of each good is equal to the wholesale price plus an exogenous markup, which markup need not be forecastable in advance.
} 
(3.13) is still correct, up to terms that are independent of policy, and the same first-order conditions continue to apply. The optimal policy rules just derived continue to be correct, except that the central bank should respond only to variation in the forecastable components of inflation and the output gap. For example, the optimal instrument rule takes the form

$i_{t}=\left(1-\rho_{1}\right) i^{*}+\rho_{1} i_{t-1}+\rho_{2} \Delta i_{t-1}+\phi_{\pi} \mathrm{E}_{t-d} F_{t}(\pi)+\frac{\phi_{x}}{4} \mathrm{E}_{t-d} F_{t}(x)-\theta_{\pi} \mathrm{E}_{t-d-1} \pi_{t-1}-\frac{\theta_{x}}{4} \mathrm{E}_{t-d} x_{t-1}$

instead of (3.16), and even when $\gamma=0$ the central bank should respond to forecasts of inflation and output $d$ periods in advance, rather than to current inflation and output.

We find that our previous conclusions about the character of optimal policy remain largely intact, even when we allow for delays in the effect of monetary policy. An optimal instrument rule still involves interest-rate inertia to exactly the same degree as was determined earlier; in particular, the optimal rule is super-inertial for all possible values of the parameters. We also find once again that optimal policy is only modestly forward-looking. If the components of inflation and the output gap that are affected by monetary policy are determined $d$ periods in advance, it follows that policy should respond only to forecasts of inflation and the output gap $d$ or more periods in the future. However, the interest rate in any given period should be set ( $d$ periods earlier) on the basis of the projected inflation rate and output gap for the period in which the interest rate applies and periods immediately thereafter; and even when the degree of inflation inertia is substantial, interest rates should be based mainly on projections for that period and a few months farther in the future. There continues to be little support for the idea that primary emphasis should be placed on inflation forecasts for a period one to two years later than the period for which the interest rate is set.

\subsection{Imperfect Information about the State of the Economy}

Our analysis thus far has assumed that the central bank has complete information about the current state of the economy, and thus can bring about an optimal state-contingent path for its instrument, regardless of how that may require its operating target to vary in response to disturbances. In reality, central-bank information about current conditions is imperfect. 
At the time that the operating target is chosen, the current quarter's inflation rate and level of real GDP - both required to implement a rule such as (1.17) - are not yet known, and must be forecasted based on a variety of indicators. The estimates available "in real time" differ substantially from the values that are eventually determined to have been correct (Orphanides, 1998). Still less does a central bank know the current quarter's efficient level of output in real time; estimates of "potential output" can be revised by several percentage points after some years of additional data become available. A proper analysis of optimal policy needs to take account of such constraints upon the central bank's information.

The Lagrangian method used above to characterize the optimal state-contingent plan can be adapted to a situation in which the central bank has imperfect information, as shown by Svensson and Woodford (2001). One obtains similar first-order conditions, but with changes in the information sets with respect to which certain expectations are conditioned. As a simple example, consider again our basic neo-Wicksellian model, as described in section 1, but suppose now that the central bank has less information than the private sector each period. The first-order conditions (1.13) - (1.14) for the optimal state-contingent plan still apply, but (1.15) must now be replaced by

$$
\lambda_{i}\left(i_{t}-i^{*}\right)+\sigma \Xi_{1 t \mid t}=0
$$

where we use the notation $z_{t \mid t}$ for the expectation of any variable $z_{t}$ conditional upon the central bank's information set at the time that the period $t$ instrument setting is chosen. This last condition is modified in the way indicated because the central bank must choose a single instrument setting for each of the possible states at date $t$ that it is unable to distinguish on the basis of its information, though $\Xi_{1 t}$ (the shadow value to the bank of relaxing the constraint associated with the IS equation) may differ across those states.

In this case, we can no longer use (3.18) to eliminate $\Xi_{1 t}$ from the other two first-order conditions, and so we can no longer obtain an instrument rule that refers only to the evolution of the interest rate, the inflation rate and the output gap. However, we can still define a 
variable

$$
\bar{\imath}_{t} \equiv i^{*}-\frac{\sigma}{\lambda_{i}} \Xi_{1 t},
$$

indicating an ideal instrument setting that would be optimal if the central bank had the information required to implement it. Then condition (3.19) can be used with (1.13) (1.14) as before to show that this variable evolves according to a law of motion

$$
\bar{\imath}_{t}=\left(1-\rho_{1}\right) i^{*}+\rho_{1} \bar{\imath}_{t-1}+\rho_{2} \Delta \bar{\imath}_{t-1}+\phi_{\pi} \pi_{t}+\phi_{x} \Delta x_{t} / 4,
$$

where the coefficients are again defined in (1.18) - (1.19). (We can assign arbitrary initial conditions to begin this recursion, and still obtain a policy rule that is optimal from a timeless perspective.) First-order condition (3.18) then can be written simply as

$$
i_{t}=\bar{\imath}_{t \mid t} .
$$

This states that the central bank chooses an operating target for each period that equals the expected value of the ideal instrument setting conditional upon the bank's information set at the time that it must choose.

Under imperfect information, then, optimal policy can be described by the rule (3.21), where the ideal instrument setting referred to in this rule is defined by (3.20). In practice, estimation of the conditional expectation referred to in this rule will require the use of a Kalman filter, the coefficients of which depend on the law of motion of the ideal instrument setting (3.20). The coefficients of the Kalman filter also depend on the equilibrium comovement of the endogenous indicator variables with inflation and the output gap, which in turn depend on the coefficients of the Kalman filter (insofar as the central bank's policy is based on it). This fixed-point problem is discussed further in Svensson and Woodford (2001).

Svensson and Woodford show that certain equations that describe the optimal instrument setting continue to hold in the case of imperfect information, except that variables not observed by the central bank are replaced by their expectations conditional upon the bank's information set. Thus this kind of linear-quadratic policy problem possesses a certainty 
equivalence property. Nonetheless, it is important to realize that one cannot naively apply this principle to any rule that describes optimal policy in the case of full information. For example, it would not be correct to set interest rates using (1.17), simply replacing each term on the right-hand side by the central bank's estimate of the term at the time that it sets $i_{t}$. This application of certainty equivalence would suggest a rule of the form

$$
i_{t}=\left(1-\rho_{1}\right) i^{*}+\rho_{1} i_{t-1}+\rho_{2} \Delta i_{t-1}+\phi_{\pi} \pi_{t \mid t}+\frac{\phi_{x}}{4}\left(x_{t \mid t}-x_{t-1 \mid t}\right),
$$

since $i_{t-1}$ and $i_{t-2}$ must be part of the central bank's information set. But this is not an optimal rule in the case of imperfect information; instead of $i_{t-1}$ and $i_{t-2}$, the optimal rule responds to $\bar{\imath}_{t-1 \mid t}$ and $\bar{l}_{t-2 \mid t}$. These are not the same, since in general the central bank will have additional information by the time that it sets $i_{t}$ about what the correct values of $\bar{\imath}_{t-1}$ and $\bar{\imath}_{t-2}$ were, relative to its estimates at the time of its earlier interest-rate decisions. Hence the notion of "certainty equivalence" must be applied with care.

Nonetheless, optimal policy under imperfect information is in many ways similar to optimal policy under full information. For example, it is no more forward-looking than our previous results indicated; in the present example, the optimal interest-rate operating target for period $t$ depends on the projections $\pi_{t \mid t}$ and $x_{t \mid t}$, but not on projections of inflation or the output gap for any dates farther in the future. And once again, optimal policy will imply substantial persistence in interest-rate fluctuations. Taking the expectation of (3.20) conditional upon the central bank's information set at date $t-2$, one obtains

$$
i_{t \mid t-2}=\left(1-\rho_{1}\right) i^{*}+\left(\rho_{1}+\rho_{2}\right) i_{t-1 \mid t-2}-\rho_{2} i_{t-2}+\phi_{\pi} \pi_{t \mid t-2}+\frac{\phi_{x}}{4}\left(x_{t \mid t-2}-x_{t-1 \mid t-2}\right) .
$$

This in turn implies that

$$
\rho_{k+2}(i)=\left(\rho_{1}+\rho_{2}\right) \rho_{k+1}(i)-\rho_{2} \rho_{k}(i)+\phi_{\pi} \beta_{k+2}(\pi, i)+\frac{\phi_{x}}{4}\left(\beta_{k+2}(x, i)-\beta_{k+1}(x, i)\right),
$$

for each $k \geq 0$, where we use the notation

$$
\rho_{k}(z) \equiv \operatorname{corr}\left(z_{t+k}, z_{t}\right), \quad \beta_{k}(y, z) \equiv \operatorname{cov}\left(y_{t+k}, z_{t}\right) / \operatorname{var}\left(z_{t}\right)
$$


Equation (3.22) is not enough by itself to allow one to solve for the autocorrelation function of the equilibrium interest rate process $\left\{\rho_{k}(i)\right\}$; this depends on the regression coefficients $\left\{\beta_{k}(\pi, i)\right\}$ and $\left\{\beta_{k}(x, i)\right\}$, which cannot be determined from the form of the monetary policy rule alone. But (3.22) is the same restriction on the autocorrelation function as is implied by (1.17) in the full-information case; thus the fact that $\rho_{1}, \rho_{2}>1$ in (3.20) makes a high degree of serial correlation of interest rates likely in the case of imperfect information as well.

\section{Conclusions}

We have shown that robustly optimal policy rules can be constructed for each of a variety of simple forward-looking models of the monetary transmission mechanism. We have seen that these rules may take the form either of an implicit instrument rule - an interest-rate feedback rule that generalizes the one proposed by Taylor (1993) — or of a pure targeting rule - a history-dependent inflation target that generalizes the "flexible inflation targeting" rule proposed by Svensson (1999). In the cases where both representations of optimal policy are possible, these are actually equivalent policy rules, at least as far as their implications for rational-expectations equilibrium are concerned, though the policy commitment is described in apparently different ways.

Our examples offer insights into several questions posed in the introduction. First, we have seen that, at least in the case of the simple models considered here, ${ }^{48}$ optimal policy rules can be expressed in terms of a commitment to bring about a certain (time-invariant) linear relationship between the paths of the short-term nominal interest-rate instrument of the central bank, an inflation measure, and a measure of the output gap, as proposed by both Taylor and Svensson. And this is not only a possible representation of optimal policy in these cases, but one with uniquely desirable properties, discussed in detail in Giannoni

\footnotetext{
${ }^{48}$ While the models are simple, it is perhaps worth recalling that they do represent log-linearizations of completely specified intertemporal general-equilibrium models of the monetary transmission mechanism, and at least those considered in section 3 are already sophisticated enough to match a number of salient features of the econometric evidence on the effects of monetary policy shocks, as discussed in Woodford (2002, chaps. $3-4)$.
} 
and Woodford (2002). Of course, in more complex (but more realistic) models, optimal rules are likely to involve additional state variables besides these three; we have already seen an illustration of this in section 3.1, where, in general, the presence of wage as well as price stickiness implies that an optimal rule will involve responses to more than one inflation measure (wage as well as price inflation). Nonetheless, the general form of rules that have been widely discussed in the recent literature are not found to be fundamentally misguided; there is no reason why an optimal rule must pay attention to monetary aggregates, for example, or why it must explicitly respond in different ways to different types of disturbances.

An issue that is much debated by monetary economists is whether it is desirable for monetary policy to respond to a measure of the output gap, as is prescribed both by the "Taylor rule" and by common examples of "flexible inflation targeting" rules. Here we have found that our optimal policy rules all prescribe interest-rate adjustments or modification of the inflation target in response to changes in the projected path of the (correctly defined) output gap, and thus our results provide some justification for the common emphasis upon this variable. While it might also be possible to formulate policy rules consistent with an optimal equilibrium that would not involve explicit reference to this variable, such alternative representations of optimal policy would either not be robustly optimal like the rules derived here - the coefficients of the optimal rule would depend upon precise details of the assumed statistical character of the disturbances ${ }^{49}$ — or they would not be direct rules — they would involve explicit reference to variables other than the target variables, such as specific exogenous disturbances. ${ }^{50}$ Hence, insofar as a robustly optimal, direct rule is desirable, there

\footnotetext{
${ }^{49}$ For example, Woodford (1999a) derives an optimal policy rule for the model of section 1 that involves only the short-term nominal interest rate and an inflation measure, and argues that this representation of optimal policy is desirable because it can be implemented without requiring the central bank to measure the output gap. However, the rule discussed there is optimal only under a very specific assumption about the disturbances: there are no cost-push shocks, and the natural rate of interest is an $\operatorname{AR}(1)$ process, with innovations that are revealed in the same period that they affect the natural rate. Furthermore, the numerical coefficients of the optimal rule depend on the coefficient of serial correlation of the natural-rate disturbances. Instead, the rules discussed in section 1 are equally optimal under this specification of the disturbances and a vast number of other possibilities.

${ }^{50}$ For example, an optimal policy rule could be constructed for the model of section 1, under a particular specification of the statistical properties of the disturbances, by solving for the optimal state-contingent paths $\left\{\bar{\imath}_{t}, \bar{\pi}_{t}\right\}$ of the nominal interest rate and inflation, and then choosing a policy rule of the form $i_{t}=$
} 
is an important advantage to expressing the central bank's policy commitment in terms of a rule that involves the bank's estimate of (or projection of) the path of the output gap.

There are certainly substantial difficulties involved in accurate measurement of the output gap in practice. But many of these can be well-represented by additive measurement error of the kind considered by Svensson and Woodford (2001), so that certainty-equivalence applies, as discussed in section 3.3. In this case, it is still optimal to commit to a policy rule with the same coefficient on the central bank's estimate of the output gap as would be optimal under full information; the measurement error affects only the way in which it is optimal for the central bank to form its estimate of current and past output gaps. For example, an optimal estimate of the output gap will generally make use of information about wages and prices, and not simply available quantity measures. But this does not mean that it is not useful for the central bank to describe its policy commitment in terms of a relationship between the output gap and other variables. For this description of policy will be much more robust than an explicit description of the way that the central bank should respond to specific indicator variables, which will depend on the bank's current beliefs about the statistical properties of the various disturbances (including the ones responsible for the measurement problems).

It is also worth noting that the errors that have been observed historically in real-time estimates of the output gap (documented by Orphanides, 2000) have been much greater in the case of estimates of the absolute level of the output gap than in the case of estimates of the quarter-to-quarter changes in the gap. ${ }^{51}$ (Errors in the recognition of shifts in the trend rate of growth of potential output until years later have caused substantial, highly persistent $\bar{\imath}_{t}+\phi\left(\pi_{t}-\bar{\pi}_{t}\right)$, where $\phi>1$ in conformity with the "Taylor Principle". It follows from Proposition xx of Woodford (2002, chap. 4) that a rule of this kind implies a determinate rational-expectations equilibrium, and it is obvious that the rule is consistent with the optimal paths of the variables. However, such a rule involves explicit reference to the state of the world as defined by the history of exogenous disturbances, and the way in which the terms $\bar{\imath}_{t}$ and $\bar{\pi}_{t}$ vary with the history of disturbances also depends on the details of the assumed statistical properties of the disturbances.

${ }^{51}$ This assumes, as does Orphanides, that current conventional estimates of past levels of potential output are in fact correct. Of course, the conception of potential output upon which such estimates are based may not be the same one as in the "output gap" to which an optimal policy rule would respond, as suggested by Woodford (2001). But this sort of error is not an inevitable one, resulting from data limitations, but rather one that can be eliminated through clarification of the optimal rule. 
mis-estimates of the absolute gap; but this particular source of measurement error has little effect on the higher-frequency components of the output gap estimate.) But the optimal rules exhibited above all involve only the projected path of quarter-to-quarter changes in the output gap, and are independent of the absolute level of the gap, even though it is the absolute size of the gap that one wishes to stabilize. Because of this, it is less obvious that output-gap mismeasurement should be a serious problem in the case of the optimal rules derived here than under common proposals that would make policy depend on the absolute level of the current or projected future output gap.

Our analysis also offers insights into the question of which inflation measure policy should respond to or target. The answer given here is the particular measure or measures that appear as target variables in the welfare-theoretic loss function derived according to the principles set out in Woodford (2002, chap. 6), which is to say, the inflation measures that are directly related to measures of the relative-price distortions that result from imperfect synchronization of wage and price changes. The inflation measure that is correct will thus depend on the nature of the nominal rigidities associated with wage and price-setting, which is ultimately an empirical question. In our baseline model, with flexible wages and the same degree of stickiness of all goods prices, the relevant inflation rate is the change in a uniformly weighted index of goods prices, which conforms fairly closely to the kind of price index actually targeted by the central banks with inflation targets. But under other assumptions, the correct inflation measure will differ. For example, we have shown in section 3.1 that if wages as well as prices are sticky, the optimal rule must involve wage inflation as well as price inflation. Similarly, if some goods prices are sticky while others are not, the correct inflation measure will be an index of "core inflation," - an index of the changes in the prices only of the sticky-price goods, as discussed in Woodford (2002, chap. 6, sec. 4.3).

We also obtain some tentative conclusions about the degree of history-dependence of optimal policy rules. Even in our baseline model, which posits an extremely simple dynamic structure, our optimal policy rules involve substantial history-dependence of a kind not present in proposals such as those of Taylor (1993) and Svensson (1997, 1999). In addition 
to the fact that policy should respond to the projected change in the output gap rather than its level, which makes the recent past level of the output gap relevant for current policy, we find that past nominal interest rates should affect the current policy setting. Specifically, both our optimal instrument rule (1.17) and our optimal pure targeting rule (1.25) have the feature that, for any given inflation and output-gap projections, interest rates should be higher than they otherwise would be if (i) interest rates have recently been higher than average, or (ii) interest rates have recently been rising. ${ }^{52}$ Thus the optimal rules incorporate both the interest-rate persistence (a positive effect of $i_{t-1}$ on the choice of $i_{t}$ ) and interestrate momentum (a positive effect of $\Delta i_{t-1}$ on the choice of $\Delta i_{t}$ ) that characterize the actual Fed reaction functions estimated by Judd and Rudebusch (1998).

Finally, we have also explored the degree to which optimal rules should make policy a function of projections of inflation and/or output many quarters in the future. In our baseline model, it is possible to formulate a robustly optimal policy rule (the implicit instrument rule (1.17) that involves no projections farther in the future than the period for which the nominal interest-rate operating target is being set. Perhaps surprisingly, this rule is optimal regardless of what we may assume about the availability of advance information about future disturbances. Of course, this strong result depends on the purely forward-looking character of that simple model of inflation and output determination. But even when we allow for a high degree of inflation inertia, in section 2, we find that an optimal policy rule depends much more on the projected inflation rate and output gap in the quarter for which policy is being set that on the projections for any later horizons. And while projections for later quarters do matter to some extent if the degree of inflation inertia is sufficiently great, projections farther than a year in the future matter little even in this case. Thus we find little justification for a policy that gives primary attention to the inflation forecast at a horizon two years in the future, as is true of the inflation-forecast targeting currently practiced at the Bank of England.

\footnotetext{
${ }^{52}$ In the case of $(1.25)$, one observes that either a high value of $i_{t-1}$ or a high value of $\Delta i_{t-1}$ require a lower value for the output-gap-adjusted inflation forecast — that is, these conditions require policy to be tightened, though the rule itself does not specify the interest-rate setting that this involves.
} 
It is important nonetheless to stress that our results do not justify a purely backwardlooking approach to the conduct of policy. In all of the cases considered, our optimal rules are implicit rules, which is to say that they specify a criterion that must be satisfied by the central bank's projections of inflation and output given its policy. The criterion in question involves variables the values of which depend on the current policy action that is chosen; hence they must be projected using a model of the monetary transmission mechanism, rather than simply being measured. It is true that optimal policy could also be described by an explicit (purely backward-looking) instrument rule, specifying the instrument setting as a function of current exogenous disturbances and past (or at any rate predetermined) state variables, that need simply be measured. But such a representation of optimal policy would not be robust to changes in the assumed character of the disturbance processes, unlike the implicit rules derived here. Hence we would argue that the use of a quantitative model, that can be used to project the effects of prospective policy settings, is essential to the optimal conduct of monetary policy. And in a model that takes account of forward-looking private sector behavior, projections for the current quarter cannot generally be made without forecasting the economy's subsequent evolution as well.

Furthermore, in the case that spending and pricing decisions are predetermined $d$ periods in advance, as assumed in many empirical models, the optimal policy is one under which the central bank's interest-rate operating target is also chosen $d$ periods in advance, on the basis of projections of inflation and output for the period for which the interest rate is being chosen (if not projections farther in the future as well). In this case, policy decisions necessarily will depend crucially on projections of conditions at least $d$ periods in the future. However, the lag $d$ by which spending or pricing decisions are predetermined is not plausibly longer than one or two quarters. And even in this case, no justification is provided for basing the interest-rate operating target for a given period on forecasts regarding points in time that are much more distant than the period for which the interest-rate decision is being made. Hence our results provide little support for the desirability of basing interest-rate decisions primarily on forecasts of conditions as long as two years in the future. 


\section{References}

Altig, David, Lawrence J. Christiano, Martin S. Eichenbaum, and Jesper Linde, "Technology Shocks and Aggregate Fluctuations," unpublished, Federal Reserve Bank of Cleveland, 2002.

Amato, Jeffery D., and Thomas Laubach, "Estimation and Control of an OptimizationBased Model with Sticky Wages and Prices," unpublished, October 2001a. (Forthcoming, Journal of Economic Dynamics and Control.)

_- - and _- _-, "Rule-of-Thumb Behavior and Monetary Policy," unpublished, Bank for International Settlements, December 2001b.

Batini, Nicoletta, and Joe Pearlman, "Too Much Too Soon: Instability and Indeterminacy with Forward-Looking Rules," unpublished, Bank of England, July 2002.

Bernanke, Ben S., and Michael Woodford, "Inflation Forecasts and Monetary Policy," Journal of Money, Credit and Banking 24: 653-684 (1997).

Boivin, Jean, and Marc Giannoni, "Has Monetary Policy Become Less Powerful?" Staff Report no. 144, Federal Reserve Bank of New York, March 2002.

Christiano, Lawrence J., Martin S. Eichenbaum, and Charles L. Evans, "Nominal Rigidities and the Dynamic Effects of a Shock to Monetary Policy," NBER Working Paper no. 8403, July 2001.

Clarida, Richard, Jordi Gali, and Mark Gertler, "Monetary Policy Rules and Macroeconomic Stability: Evidence and Some Theory," Quarterly Journal of Economics 115: $147-180$ (2000).

Erceg, Christopher J., Dale W. Henderson, and Andrew T. Levin, "Optimal Monetary Policy with Staggered Wage and Price Contracts," Journal of Monetary Economics 46: 281-313 (2000).

Gali, Jordi, "The Conduct of Monetary Policy in the Face of Technological Change: Theory and Postwar U.S. Evidence," unpublished, Universitat Pompeu Fabra, October 2000. 
_- - , and Mark Gertler, "Inflation Dynamics: A Structural Econometric Analysis," Journal of Monetary Economics 44: 195-222 (1999).

Giannoni, Marc P., "Optimal Interest-Rate Rules in a Forward-Looking Model, and Inflation Stabilization versus Price-Level Stabilization," unpublished, Princeton University, 2001.

_- - , and Michael Woodford, "Optimal Interest-Rate Rules: I. General Theory," unpublished, Princeton University, August 2002.

Hall, Robert E., "Monetary Strategy with an Elastic Price Standard," in Price Stability and Public Policy, Kansas City: Federal Reserve Bank of Kansas City, 1984.

Judd, John F., and Glenn D. Rudebusch, "Taylor's Rule and the Fed: 1970-1997," Federal Reserve Bank of San Francisco Economic Review, 1998(3), pp. 3-16.

Levin, Andrew, Volker Wieland, and John C. Williams, "The Performance of ForecastBased Monetary Policy Rules under Model Uncertainty," FEDS paper no. 2001-39, Federal Reserve Board, August 2001.

McCallum, Bennett T., "Should Monetary Policy Respond Strongly to the Output Gaps?" NBER Working Paper no. 8226, April 2001.

McCallum, Bennett T., and Edward Nelson, "Performance of Operational Policy Rules in an Estimated Semi-Classical Structural Model," in J.B. Taylor, ed., Monetary Policy Rules, Chicago: University of Chicago Press, 1999.

Orphanides, Athanasios, "Monetary Policy Evaluation with Noisy Information," FEDS paper no. 1998-50, Federal Reserve Board, October 1998.

_- - "The Quest for Prosperity without Inflation," Working Paper no. 15, European Central Bank, March 2000.

Rotemberg, Julio J., and Michael Woodford, "An Optimization-Based Econometric Framework for the Evaluation of Monetary Policy," NBER Macroeconomics Annual 12: 297-346 (1997).

_- - and - - "Interest-Rate Rules in an Estimated Sticky-Price Model," in J.B. 
Taylor, ed., Monetary Policy Rules, Chicago: University of Chicago Press, 1999. Sbordone, Argia M., "Prices and Unit Labor Costs: A New Test of Price Stickiness," Journal of Monetary Economics 49: 265-292 (2002a).

_- - - "An Optimizing Model of U.S. Wage and Price Dynamics," unpublished, Rutgers University, February 2002b.

Smets, Frank, and Raf Wouters, "An Estimated Dynamic Stochastic General Equilibrium Model of the Euro Area," unpublished, European Central Bank, May 2002.

Steinsson, Jon, "Optimal Monetary Policy in an Economy with Inflation Persistence," Working Paper no. 11, Central Bank of Iceland, December 2000.

Svensson, Lars E.O., "Inflation Forecast Targeting: Implementing and Monitoring Inflation Targets," European Economic Review 41: 1111-1146 (1997).

_- - - "Inflation Targeting as a Monetary Policy Rule," Journal of Monetary Economics 43: 607-654 (1999).

_- - , and Michael Woodford, "Implementing Optimal Policy through Inflation-Forecast Targeting," unpublished, Princeton University, November 1999.

- - - and - - " "Indicator Variables for Optimal Policy under Asymmetric Information," NBER Working Paper no. 8255, April 2001. (Forthcoming, Journal of Economic Dynamics and Control.)

Taylor, John B., "Discretion versus Policy Rules in Practice," Carnegie-Rochester Conference Series on Public Policy 39: 195-214 (1993).

Woodford, Michael, "Optimal Monetary Policy Inertia," NBER Working Paper no. 7261, August 1999a.

_- _- "Commentary: How Should Monetary Policy Be Conducted in an Era of Price Stability?" in New Challenges for Monetary Policy, Kansas City: Federal Reserve Bank of Kansas City, 1999b.

_- _- "The Taylor Rule and Optimal Monetary Policy," American Economic Review 91(2): 232-237 (2001). 
- - - Interest and Prices: Foundations of a Theory of Monetary Policy, unpublished, Princeton University, July 2002. 


\section{A Proofs of Propositions}

\section{A.1 The Optimal Non-Inertial Plan}

In the case that the disturbances are of the form (1.6) - (1.7), the optimal non-inertial plan is given by

$$
z_{t}=\bar{z}+F e_{t}, \quad i_{t}=\bar{\imath}+f_{i} e_{t}
$$

where $z_{t} \equiv\left[\pi_{t}, x_{t}\right]^{\prime}, e_{t} \equiv\left[\hat{r}_{t}^{n}, u_{t}\right]^{\prime}$. The long-run average values $\bar{z}, \bar{\imath}$, and the response coefficients are given by

$$
\begin{aligned}
& \bar{z}=\left[\begin{array}{c}
\frac{(1-\beta) \kappa^{-1} \lambda_{x} x^{*}+\lambda_{i}\left(i^{*}-\bar{r}\right)}{1+(1-\beta)^{2} \kappa^{-2} \lambda_{x}+\lambda_{i}} \\
\frac{1-\beta}{\kappa} \frac{(1-\beta) \kappa^{-1} \lambda_{x} x^{*}+\lambda_{i}\left(i^{*}-\bar{r}\right)}{1+(1-\beta)^{2} \kappa^{-2} \lambda_{x}+\lambda_{i}}
\end{array}\right] \\
& \bar{\imath}=\frac{(1-\beta) \kappa^{-1} \lambda_{x} x^{*}+\lambda_{i}\left(i^{*}-\bar{r}\right)}{1+(1-\beta)^{2} \kappa^{-2} \lambda_{x}+\lambda_{i}}+\bar{r}
\end{aligned}
$$

and

$$
F=\left[\begin{array}{ll}
\pi_{r} & \pi_{u} \\
x_{r} & x_{u}
\end{array}\right], \quad f_{i}=\left[\begin{array}{ll}
i_{r} & i_{u}
\end{array}\right]
$$

where the coefficients

$$
\begin{aligned}
\pi_{r} & =\frac{\lambda_{i} \sigma^{-1}\left(\gamma_{r}-\rho_{r} \kappa \sigma\right) \kappa}{h_{r}}, \quad \pi_{u}=\frac{\lambda_{i} \sigma^{-2}\left(\gamma_{u}-\rho_{u} \kappa \sigma\right)\left(1-\rho_{u}\right)+\xi_{u}}{h_{u}} \\
x_{r} & =\frac{\lambda_{i} \sigma^{-1}\left(\gamma_{r}-\rho_{r} \kappa \sigma\right)\left(1-\beta \rho_{r}\right)}{h_{r}}, \quad x_{u}=\frac{\rho_{u} \lambda_{i} \sigma^{-1}\left(\gamma_{u}-\rho_{u} \kappa \sigma\right)-\kappa}{h_{u}} \\
i_{r} & =\frac{\xi_{r}\left(1-\beta \rho_{r}\right)+\kappa^{2}}{h_{r}}>0, \quad i_{u}=\frac{\sigma^{-1} \kappa\left(1-\rho_{u}\right)+\xi_{u} \rho_{u}}{h_{u}}>0,
\end{aligned}
$$

and

$$
\begin{aligned}
\gamma_{j} & \equiv\left(1-\rho_{j}\right)\left(1-\beta \rho_{j}\right)>0 \\
h_{j} & \equiv \lambda_{i} \sigma^{-2}\left(\gamma_{j}-\rho_{j} \kappa \sigma\right)^{2}+\lambda_{x}\left(1-\beta \rho_{j}\right)^{2}+\kappa^{2}>0 \\
\xi_{j} & \equiv \lambda_{x}\left(1-\beta \rho_{j}\right)>0,
\end{aligned}
$$

for $j \in\{r, u\}$. 


\section{A.2 Proposition 4}

Proposition 4. Let $\kappa, \sigma, \lambda_{i}, \lambda_{x}>0,0<\beta<1,0 \leq \rho_{r}<1$, and $0 \leq \rho_{u}<1$. Suppose the disturbances are of the form (1.6) - (1.7), with autocorrelation coefficients satisfying the bounds

$$
\begin{aligned}
0 & <\left(1-\rho_{r}\right)\left(1-\beta \rho_{r}\right)-\rho_{r} \kappa \sigma \\
& \leq\left(1-\rho_{u}\right)\left(1-\beta \rho_{u}\right)-\rho_{u} \kappa \sigma<\frac{\kappa \sigma}{\lambda_{i}} .
\end{aligned}
$$

Then (1.8) defines a Taylor rule of the form (1.5) with coefficients $\phi_{\pi}>1, \phi_{x}>0$. Furthermore, commitment to this rule implies a determinate rational expectations equilibrium, which implements the optimal non-inertial plan.

Proof: The instrument rule (1.8) is of the form of the Taylor rule (1.5), where

$$
\left[\begin{array}{ll}
\phi_{\pi} & \phi_{x} / 4
\end{array}\right]=f_{i} F^{-1}=\left[\begin{array}{ll}
\frac{i_{u} x_{r}-i_{r} x_{u}}{\pi_{u} x_{r}-\pi_{r} x_{u}} & \frac{i_{r} \pi_{u}-i_{u} \pi_{r}}{\pi_{u} x_{r}-\pi_{r} x_{u}}
\end{array}\right]
$$

Under the assumption of the proposition, we have $\pi_{r}>0, \pi_{u}>0, x_{r}>0, x_{u}<0, i_{r}>0$, and $i_{u}>0$. It follows that

$$
\pi_{u} x_{r}-\pi_{r} x_{u}>0
$$

Furthermore,

$$
\begin{aligned}
i_{r} \pi_{u}-i_{u} \pi_{r}= & \frac{\left(\lambda_{i} \sigma^{-2}\left(\gamma_{u}-\rho_{u} \kappa \sigma\right)\left(1-\rho_{u}\right)+\xi_{u}\right)\left(\xi_{r}\left(1-\beta \rho_{r}\right)+\kappa^{2}\right)}{h_{r} h_{u}} \\
& -\frac{\lambda_{i} \sigma^{-1}\left(\gamma_{r}-\rho_{r} \kappa \sigma\right) \kappa\left(\sigma^{-1} \kappa\left(1-\rho_{u}\right)+\rho_{u} \xi_{u}\right)}{h_{r} h_{u}} \\
= & \left(\chi_{1}+\lambda_{i} \sigma^{-2} \chi_{2}\right)\left(h_{r} h_{u}\right)^{-1}
\end{aligned}
$$

where

$$
\begin{aligned}
& \chi_{1} \equiv \lambda_{i} \sigma^{-2} \kappa^{2}\left(\gamma_{u}-\gamma_{r}+\left(\rho_{r}-\rho_{u}\right) \kappa \sigma\right)\left(1-\rho_{u}\right)+\xi_{u}\left(\xi_{r}\left(1-\beta \rho_{r}\right)+\kappa^{2}\right) \\
& \chi_{2} \equiv \xi_{r}\left(\gamma_{u}-\rho_{u} \kappa \sigma\right)\left(1-\rho_{u}\right)\left(1-\beta \rho_{r}\right)-\xi_{u}\left(\gamma_{r}-\rho_{r} \kappa \sigma\right) \rho_{u} \kappa \sigma
\end{aligned}
$$


and

$$
\begin{aligned}
\gamma_{j} & \equiv\left(1-\rho_{j}\right)\left(1-\beta \rho_{j}\right)>0 \\
h_{j} & \equiv \lambda_{i} \sigma^{-2}\left(\gamma_{j}-\rho_{j} \kappa \sigma\right)^{2}+\lambda_{x}\left(1-\beta \rho_{j}\right)^{2}+\kappa^{2}>0 \\
\xi_{j} & \equiv \lambda_{x}\left(1-\beta \rho_{j}\right)>0
\end{aligned}
$$

for $j \in\{r, u\}$. Note that (A.1) and (A.2) imply $\rho_{r} \geq \rho_{u}, \gamma_{u} \geq \gamma_{r}$, so that $\chi_{1}>0$. Moreover, given that $\left(1-\rho_{u}\right)\left(1-\beta \rho_{r}\right)-\rho_{u} \kappa \sigma>0$, we have

$$
\begin{aligned}
\chi_{2} & =\xi_{r}\left(\gamma_{u}\left(1-\rho_{u}\right)\left(1-\beta \rho_{r}\right)-\rho_{u} \kappa \sigma\left(1-\rho_{u}\right)\left(1-\beta \rho_{r}\right)\right)-\xi_{u}\left(\gamma_{r}-\rho_{r} \kappa \sigma\right) \rho_{u} \sigma \kappa \\
& \geq \xi_{r}\left(\gamma_{u} \gamma_{r}-\rho_{u} \kappa \sigma \gamma_{u}\right)-\xi_{u}\left(\gamma_{r}-\rho_{r} \kappa \sigma\right) \rho_{u} \sigma \kappa \\
& \geq \xi_{r} \gamma_{u}\left(\gamma_{r}-\rho_{r} \kappa \sigma\right)-\xi_{u}\left(\gamma_{r}-\rho_{r} \kappa \sigma\right) \rho_{u} \sigma \kappa \\
& =\xi_{u}\left(\left(1-\beta \rho_{r}\right)\left(1-\rho_{u}\right)-\rho_{u} \sigma \kappa\right)\left(\gamma_{r}-\rho_{r} \kappa \sigma\right) \\
& \geq \xi_{u}\left(\gamma_{r}-\rho_{r} \sigma \kappa\right)\left(\gamma_{r}-\rho_{r} \kappa \sigma\right) \\
& =\xi_{u}\left(\gamma_{r}-\rho_{r} \sigma \kappa\right)^{2}>0 .
\end{aligned}
$$

It follows that $\phi_{x}>0$.

We now show that $\phi_{\pi}>1$. Using (A.3), we have

$$
\phi_{\pi}-1=\frac{N_{\pi}}{h_{r} h_{u}\left(\pi_{u} x_{r}-\pi_{r} x_{u}\right)},
$$

where

$$
\begin{aligned}
N_{\pi}= & \left(\sigma^{-1} \kappa\left(1-\rho_{u}\right)+\xi_{u} \rho_{u}\right)\left(\lambda_{i} \sigma^{-1}\left(\gamma_{r}-\rho_{r} \kappa \sigma\right)\left(1-\beta \rho_{r}\right)\right) \\
& -\left(\xi_{r}\left(1-\beta \rho_{r}\right)+\kappa^{2}\right)\left(\rho_{u} \lambda_{i} \sigma^{-1}\left(\gamma_{u}-\rho_{u} \kappa \sigma\right)-\kappa\right)-\lambda_{i} \sigma^{-1}\left(\gamma_{r}-\rho_{r} \kappa \sigma\right) \\
& \times\left(\lambda_{i} \sigma^{-2}\left(\gamma_{u}-\rho_{u} \kappa \sigma\right)\left(\left(1-\rho_{u}\right)\left(1-\beta \rho_{r}\right)-\rho_{u} \kappa \sigma\right)+\xi_{u}\left(1-\beta \rho_{r}\right)+\kappa^{2}\right) .
\end{aligned}
$$

The numerator $N_{\pi}$ can be viewed as the quadratic polynomial

$$
N_{\pi}\left(\lambda_{i}\right) \equiv n_{2} \lambda_{i}^{2}+n_{1} \lambda_{i}+n_{0}
$$


where

$$
\begin{aligned}
n_{2}= & -\sigma^{-3}\left(\gamma_{r}-\rho_{r} \kappa \sigma\right)\left(\gamma_{u}-\rho_{u} \kappa \sigma\right)\left(\left(1-\rho_{u}\right)\left(1-\beta \rho_{r}\right)-\rho_{u} \kappa \sigma\right)<0 \\
n_{1}= & \sigma^{-1}\left(\left(\sigma^{-1} \kappa-\xi_{u}\right)\left(1-\beta \rho_{r}\right)\left(1-\rho_{u}\right)-\kappa^{2}\right)\left(\gamma_{r}-\rho_{r} \kappa \sigma\right) \\
& -\rho_{u} \sigma^{-1}\left(\xi_{r}\left(1-\beta \rho_{r}\right)+\kappa^{2}\right)\left(\gamma_{u}-\rho_{u} \kappa \sigma\right) \\
n_{0}= & \left(\xi_{r}\left(1-\beta \rho_{r}\right)+\kappa^{2}\right) \kappa>0 .
\end{aligned}
$$

Since $N_{\pi}\left(\lambda_{i}\right)$ is concave in $\lambda_{i}$, and satisfies $N_{\pi}(0)>0, N_{\pi}(+\infty)=-\infty$, we know that $N_{\pi}\left(\lambda_{i}\right)$ is positive for $\lambda_{i}>0$ small enough, and negative for any $\lambda_{i}$ larger than a certain critical value. Furthermore,

$$
\begin{aligned}
N_{\pi}\left(\frac{\kappa \sigma}{\gamma_{u}-\rho_{u} \kappa \sigma}\right)= & \kappa^{3}\left(1-\rho_{u}\right)\left(1-\frac{\gamma_{r}-\rho_{r} \kappa \sigma}{\gamma_{u}-\rho_{u} \kappa \sigma}\right) \\
& +\frac{\kappa \xi_{r}\left(1-\rho_{u}\right)}{\gamma_{u}-\rho_{u} \kappa \sigma}\left(\left(1-\beta \rho_{r}\right)\left(1-\beta \rho_{u}\right)+\kappa \sigma\right)\left(\rho_{r}-\rho_{u}\right) \\
\geq & 0 .
\end{aligned}
$$

Thus $N_{\pi}\left(\lambda_{i}\right)>0$ for any $\lambda_{i}$ satisfying $0<\lambda_{i}<\frac{\kappa \sigma}{\gamma_{u}-\rho_{u} \kappa \sigma}$, or equivalently for any $\lambda_{i}>0$ satisfying (A.2). It follows that $\phi_{\pi}>1$ under the conditions of the proposition.

We finally show that commitment to the rule (1.8) implies a determinate rational expectations equilibrium, which implements the optimal non-inertial plan. First note that the system of equations given by the structural equation (1.1), (1.2), and the policy rule (1.5) can be written in matrix form as

$$
\bar{I}\left[\begin{array}{c}
Z_{t+1} \\
\mathrm{E}_{t} z_{t+1} \\
\mathrm{E}_{t} i_{t+1}
\end{array}\right]=\left[\begin{array}{c}
0 \\
-\bar{\phi}
\end{array}\right]+\bar{A}\left[\begin{array}{c}
Z_{t} \\
z_{t} \\
i_{t}
\end{array}\right]+\bar{C} s_{t},
$$

where $z_{t} \equiv\left[\pi_{t}, x_{t}\right]^{\prime}, s_{t} \equiv\left[r_{t}^{n}, u_{t}\right]^{\prime}$, and

$$
\bar{I}=\left[\begin{array}{lll}
\sigma & 1 & 0 \\
\beta & 0 & 0 \\
0 & 0 & 0
\end{array}\right], \quad \bar{A}=\left[\begin{array}{lll}
0 & 1 & \sigma \\
1 & -\kappa & 0 \\
\phi_{\pi} & \phi_{x} / 4 & -1
\end{array}\right]
$$

As discussed in Giannoni and Woodford (2002, sec. 2), the equilibrium is determinate if the 
characteristic polynomial

$$
\begin{aligned}
p(\mu) & \equiv \operatorname{det}[\bar{A}-\mu \bar{I}] \\
& =\mu^{2} \beta-\left(1+\beta+\kappa \sigma+\beta \sigma \frac{\phi_{x}}{4}\right) \mu+1+\sigma \frac{\phi_{x}}{4}+\kappa \sigma \phi_{\pi}
\end{aligned}
$$

has exactly $n_{Z}=0$ roots $|\mu|<1$, or equivalently, if it has both roots outside the unit circle. Next, since the policy rule (1.8) is of the form of (1.5) with $\phi_{\pi}>1, \phi_{x}>0$, a commitment to the rule (1.8) implies that the characteristic polynomial $p(\mu)$ has the following properties

$$
\begin{aligned}
p(0) & =1+\sigma \frac{\phi_{x}}{4}+\phi_{\pi} \sigma \kappa>1 \\
p(-1) & =\left(2+\frac{\phi_{x}}{4} \sigma\right)(1+\beta)+\left(1+\phi_{\pi}\right) \kappa \sigma>0 \\
p(1) & =\sigma \kappa\left(\phi_{\pi}-1\right)+\sigma \frac{\phi_{x}}{4}(1-\beta)>0
\end{aligned}
$$

Using Proposition 1 of Woodford (2002, chap. 4), we know that $p(\mu)$ has both roots outside the unit circle if

$$
p(0)>1, \quad p(-1)>0, \quad \text { and } \quad p(1)>0 .
$$

Since these inequalities are satisfied under a commitment to the policy rule $(1.8), p(\mu)$ has both roots outside the unit circle. It follows that a commitment to the rule (1.8) implies a determinate rational expectations equilibrium, which implements the optimal non-inertial plan.

\section{A.3 Proposition 5}

Proposition 5. Suppose that $\kappa, \sigma, \lambda_{i}, \lambda_{x}>0,0<\beta<1$, and $0<\rho<1$. Then for all forecast horizons $k$ longer than some critical value, the rule of the form (1.9) that is consistent with the optimal non-inertial plan implies indeterminacy of rational-expectations equilibrium.

Proof: The structural equations (1.1) and (1.2) can be written in matrix form as following

$$
\hat{I} \mathrm{E}_{t} z_{t+1}=A z_{t}+B i_{t}+C s_{t}
$$


where $z_{t} \equiv\left[\pi_{t}, x_{t}\right]^{\prime}, s_{t} \equiv\left[r_{t}^{n}, u_{t}\right]^{\prime}$, and

$$
\hat{I} \equiv\left[\begin{array}{cc}
\sigma & 1 \\
\beta & 0
\end{array}\right], \quad A \equiv\left[\begin{array}{cc}
0 & 1 \\
1 & -\kappa
\end{array}\right], \quad B \equiv\left[\begin{array}{l}
\sigma \\
0
\end{array}\right], \quad C \equiv\left[\begin{array}{cc}
-\sigma & 0 \\
0 & -1
\end{array}\right] .
$$

The policy rule (1.9) can also be written as

$$
i_{t}=\phi_{z}^{\prime} \mathrm{E}_{t} z_{t+k}+\left(\bar{\imath}-\phi_{z}^{\prime} \bar{z}\right) .
$$

where $\phi_{z}^{\prime} \equiv\left[\begin{array}{ll}\phi_{\pi} & \phi_{x} / 4\end{array}\right]$. Combining (A.6) and (A.7), we obtain

$$
-B \phi_{z}^{\prime} \mathrm{E}_{t} z_{t+k}+\hat{I} \mathrm{E}_{t} z_{t+1}=A z_{t}+C s_{t}+B\left(\bar{\imath}-\phi_{z}^{\prime} \bar{z}\right)
$$

or, in matrix form,

$$
\bar{I} \mathrm{E}_{t}\left[\begin{array}{c}
\mathrm{E}_{t+1} z_{t+k} \\
\vdots \\
\mathrm{E}_{t+1} z_{t+2} \\
z_{t+1}
\end{array}\right]=\bar{A}\left[\begin{array}{c}
\mathrm{E}_{t} z_{t+k-1} \\
\vdots \\
\mathrm{E}_{t} z_{t+1} \\
z_{t}
\end{array}\right]+\bar{C} s_{t}+\left[\begin{array}{c}
B\left(\bar{\imath}-\phi_{z}^{\prime} \bar{z}\right) \\
0
\end{array}\right]
$$

where $\bar{I}$ and $\bar{A}$ are $2 k \times 2 k$ matrices, and $\bar{C}$ is a $2 k \times 2$ matrix satisfying

$$
\bar{I} \equiv\left[\begin{array}{ccccc}
-B \phi_{z}^{\prime} & 0 & \cdots & 0 & \hat{I} \\
0 & I_{2} & 0 & \cdots & 0 \\
\vdots & \ddots & \ddots & \ddots & \vdots \\
\vdots & & \ddots & \ddots & 0 \\
0 & \cdots & \cdots & 0 & I_{2}
\end{array}\right], \quad \bar{A} \equiv\left[\begin{array}{ccccc}
0 & \cdots & \cdots & 0 & A \\
I_{2} & 0 & \cdots & \cdots & 0 \\
0 & \ddots & \ddots & & \vdots \\
\vdots & \ddots & \ddots & \ddots & \vdots \\
0 & \cdots & 0 & I_{2} & 0
\end{array}\right] \quad \bar{C} \equiv\left[\begin{array}{c}
C \\
0 \\
\vdots \\
\vdots \\
0
\end{array}\right]
$$

Since all variables entering the vector $\left[\mathrm{E}_{t} z_{t+k-1}^{\prime}, \ldots, z_{t}^{\prime}\right]^{\prime}$ are non-predetermined, the dynamic system (A.8) admits a unique bounded solution if and only if all of the $2 k$ roots $\mu$ of the characteristic equation

$$
\operatorname{det}[\bar{I}-\mu \bar{A}]=0
$$

are inside the unit circle.

Characteristic polynomial. First note that the characteristic polynomial in our model is given by

$$
\operatorname{det}[\bar{I}-\mu \bar{A}]=\left|\begin{array}{ccccc}
-B \phi_{z}^{\prime} & 0 & \cdots & 0 & \hat{I}-\mu A \\
-\mu I_{2} & I_{2} & 0 & \cdots & 0 \\
0 & \ddots & \ddots & \ddots & \vdots \\
\vdots & \ddots & \ddots & \ddots & 0 \\
0 & \cdots & 0 & -\mu I_{2} & I_{2}
\end{array}\right|
$$


Since the determinant of any matrix remains unchanged if a multiple of one column is added to another column, we multiply each of the final block of two columns by $\mu$ and add it to the next-to-last block, to obtain

$$
\operatorname{det}[\bar{I}-\mu \bar{A}]=\left|\begin{array}{ccccc}
-B \phi_{z}^{\prime} & 0 & \ldots & \mu(\hat{I}-\mu A) & \hat{I}-\mu A \\
-\mu I_{2} & I_{2} & 0 & \ldots & 0 \\
0 & \ddots & \ddots & \ddots & \vdots \\
\vdots & \ddots & -\mu I_{2} & \ddots & 0 \\
0 & \cdots & 0 & 0 & I_{2}
\end{array}\right|
$$

We then multiply each of the block of this next-to-last block by $\mu$, and add it to the previous block, and so on, to get finally

$$
\operatorname{det}[\bar{I}-\mu \bar{A}]=\left|\begin{array}{ll}
D_{11} & D_{12} \\
D_{21} & D_{22}
\end{array}\right|
$$

where

$$
\begin{aligned}
& D_{11} \equiv \mu^{k-1}(\hat{I}-\mu A)-B \phi_{z}^{\prime}, \quad D_{12} \equiv\left[\mu^{k-2}(\hat{I}-\mu A) \quad \cdots \quad \mu(\hat{I}-\mu A) \quad \hat{I}-\mu A\right], \\
& D_{21} \equiv 0_{2(k-1) \times 2}, \quad D_{22} \equiv I_{2(k-1)} \text {. }
\end{aligned}
$$

Given that $\left|D_{22}\right| \neq 0$, we have

$$
\begin{aligned}
\operatorname{det}[\bar{I}-\mu \bar{A}] & =\left|D_{22}\right| \cdot\left|D_{11}-D_{12} D_{22}^{-1} D_{21}\right| \\
& =\left|D_{11}\right| \\
& =-\mu^{k-1} p(\mu),
\end{aligned}
$$

where

$$
p(\mu) \equiv \mu^{k+1}-(1+\beta+\kappa \sigma) \mu^{k}+\beta \mu^{k-1}+\left(\kappa \phi_{\pi}+\phi_{x} / 4\right) \sigma \mu-\phi_{x} \beta \sigma / 4 .
$$

The characteristic polynomial $\operatorname{det}[\bar{I}-\mu \bar{A}]$ has $k-1$ roots $\mu_{k+2}=\mu_{k+3}=\ldots=\mu_{2 k}=0$. The system (A.8) has a unique bounded solution if and only if the $k+1$ roots of $p(\mu)$, denoted $\mu_{1}, \mu_{2}, \ldots, \mu_{k+1}$, also lie inside the unit circle. Note that for any polynomial of the form (A.9), the product of its roots satisfies

$$
\left|\prod_{j=1}^{k+1} \mu_{k}\right|=\left|\frac{\phi_{x} \beta \sigma}{4}\right| .
$$


Thus a necessary condition for the system (A.8) to have a unique bounded solution is

$$
\left|\phi_{x} \beta \sigma / 4\right|<1 .
$$

Alternatively, if (A.10) is violated for some $k$, then $p(\mu)$ must have at least one root outside the unit circle, so that the dynamic system (A.8) implies indeterminacy of the REE.

Optimal non-inertial plan. As indicated in the text, the unique rule that is consistent with the optimal non-inertial plan (in the case that $\rho_{r}=\rho_{u}=\rho \in[0,1]$ ), is given by (1.10), where $f_{i} F^{-1}$ is independent of $k$. It follows that

$$
\phi_{z}^{\prime}=\left[\phi_{\pi}, \phi_{x} / 4\right]=\rho^{-k} f_{i} F^{-1} .
$$

We now consider three possible cases.

Case 1: optimal coefficient $\phi_{x} \neq 0$. Suppose first that $f_{i} F^{-1}[0,1]^{\prime} \neq 0$. It follows that the optimal coefficient $\phi_{x} / 4=\rho^{-k} f_{i} F^{-1}[0,1]^{\prime} \neq 0$ for all $k$, and that $\left|\phi_{x} \beta \sigma / 4\right|$ is an increasing function in $k$. Hence there exists a $\bar{k} \geq 1$ such that for any $k \geq \bar{k}$, the condition (A.10) is violated, and thus implies indeterminacy of the REE.

Case 2: optimal coefficients $\phi_{\pi} \neq 0, \phi_{x}=0$. Suppose instead that in the optimal non-inertial plan, $f_{i} F^{-1}[0,1]^{\prime}=0$. In this case, the optimal coefficient $\phi_{x}=0$ for all $k$, and the characteristic polynomial $\operatorname{det}[\bar{I}-\mu \bar{A}]$ reduces to

$$
\operatorname{det}[\bar{I}-\mu \bar{A}]=-\mu^{k} \tilde{p}(\mu)
$$

where

$$
\tilde{p}(\mu) \equiv \mu^{k}-(1+\beta+\kappa \sigma) \mu^{k-1}+\beta \mu^{k-2}+\phi_{\pi} \kappa \sigma
$$

As before, the system (A.8) has a unique bounded solution if and only if the $k$ roots of $\tilde{p}(\mu)$, denoted $\mu_{1}, \mu_{2}, \ldots, \mu_{k}$, lie inside the unit circle. A necessary condition for the system (A.8) to have a unique bounded solution is

$$
\left|\prod_{j=1}^{k} \mu_{k}\right|=\left|\phi_{\pi} \kappa \sigma\right|<1 .
$$


Suppose that $f_{i} F^{-1}[1,0]^{\prime} \neq 0$. It follows that the optimal coefficient $\phi_{\pi}=\rho^{-k} f_{i} F^{-1}[1,0]^{\prime} \neq 0$ for all $k$, and that $\left|\phi_{\pi} \kappa \sigma\right|$ is an increasing function in $k$. Hence there exists a $\bar{k} \geq 1$ such that for any $k \geq \bar{k}$, the condition (A.11) is violated, and thus implies indeterminacy of the REE.

Case 3: optimal coefficients $\phi_{\pi}=\phi_{x}=0$. Suppose finally that in the optimal noninertial plan, $f_{i} F^{-1}=[0,0]$. In this case, the optimal coefficient $\phi_{\pi}=\phi_{x}=0$ for all $k$, and the characteristic polynomial $\operatorname{det}[\bar{I}-\mu \bar{A}]$ reduces to

$$
\operatorname{det}[\bar{I}-\mu \bar{A}]=-\mu^{2 k-2} \hat{p}(\mu),
$$

where

$$
\hat{p}(\mu) \equiv \mu^{2}-(1+\beta+\kappa \sigma) \mu+\beta .
$$

Since $\hat{p}(0)=\beta>0, \hat{p}(1)=-\kappa \sigma<0$, and $\hat{p}(+\infty)=+\infty$, the polynomial $\hat{p}(\mu)$ admits one root inside and one root outside the unit circle. This implies indeterminacy of the REE.

\section{A.4 Proposition 6}

Proposition 6. Suppose that a bounded optimal state-contingent plan exists. Then in the case of any parameter values $\sigma, \kappa, \lambda_{x}, \lambda_{i}>0$ and $0<\beta<1$, a commitment to the rule described by (1.17) - (1.19) implies a determinate rational-expectations equilibrium.

Proof: The system of equations given by the structural equations (1.1), (1.2) and the policy rule $(1.17)$ - (1.19) can be written in matrix form as in (A.5), where $z_{t} \equiv\left[\pi_{t}, x_{t}\right]^{\prime}$, $Z_{t} \equiv\left[x_{t-1}, i_{t-1}, i_{t-2}\right]^{\prime}, s_{t} \equiv\left[r_{t}^{n}, u_{t}\right]^{\prime}$, and

$$
\bar{I}=\left[\begin{array}{llllll}
1 & 0 & 0 & 0 & 0 & 0 \\
0 & 1 & 0 & 0 & 0 & 0 \\
0 & 0 & 1 & 0 & 0 & 0 \\
0 & 0 & 0 & \sigma & 1 & 0 \\
0 & 0 & 0 & \beta & 0 & 0 \\
0 & 0 & 0 & 0 & 0 & 0
\end{array}\right], \quad \bar{A}=\left[\begin{array}{llllll}
0 & 0 & 0 & 0 & 1 & 0 \\
0 & 0 & 0 & 0 & 0 & 1 \\
0 & 1 & 0 & 0 & 0 & 0 \\
0 & 0 & 0 & 0 & 1 & \sigma \\
0 & 0 & 0 & 1 & -\kappa & 0 \\
-\frac{\sigma \lambda_{x}}{\lambda_{i}} & 1+\frac{\kappa \sigma}{\beta}+\beta^{-1} & -\beta^{-1} & \frac{\kappa \sigma}{\lambda_{i}} & \frac{\sigma \lambda_{x}}{\lambda_{i}} & -1
\end{array}\right] .
$$

As discussed in the subsection 2.1, the equilibrium is determinate if the characteristic polynomial $\operatorname{det}[\bar{A}-\mu \bar{I}]$ has exactly $n_{Z}=3$ roots such that $|\mu|<1$. Recall that if there are 
fewer such roots, there is no bounded solution at all. Since the rule (1.17) - (1.19) is derived from the first-order conditions $(1.13)$ - (1.15), it must be consistent with the optimal statecontingent plan. Because we assume that a bounded optimal state-contingent plan exists, it must be the case that $\operatorname{det}[\bar{A}-\mu \bar{I}]$ admits at least 3 roots inside the unit circle.

Note that we can rewrite the characteristic polynomial as

$$
\operatorname{det}[\bar{A}-\mu \bar{I}]=-p(\mu) \beta \mu
$$

where

$$
p(\mu) \equiv \mu^{4}-a \mu^{3}+b \mu^{2}-a \beta^{-1} \mu+\beta^{-2}
$$

and

$$
\begin{aligned}
& a=2 \frac{1+\beta+\sigma \kappa}{\beta}+\frac{\sigma^{2} \lambda_{x}}{\lambda_{i}} \\
& b=\frac{1+2 \kappa \beta \sigma+2 \sigma \kappa+\sigma^{2} \kappa^{2}+4 \beta+\beta^{2}}{\beta^{2}}+\sigma^{2} \frac{(1+\beta) \lambda_{x}+\kappa^{2}}{\beta \lambda_{i}}
\end{aligned}
$$

We can furthermore express $p(\mu)$ as

$$
p(\mu)=\left(\mu-\mu_{1}\right)\left(\mu-\mu_{2}\right)\left(\mu-\mu_{3}\right)\left(\mu-\mu_{4}\right)
$$

where, because of the symmetry in (A.13), the four roots $\mu_{i}$ satisfy

$$
\mu_{1}=\left(\beta \mu_{2}\right)^{-1}, \quad \text { and } \quad \mu_{3}=\left(\beta \mu_{4}\right)^{-1}
$$

Because $\operatorname{det}[\bar{A}-\mu \bar{I}]$ admits at least 3 roots inside the unit circle, (A.12) and (A.13) imply that $p(\mu)$ admits either two, three, or four roots inside the unit circle. Let us consider each case in turn:

- Let us suppose first, as a way of contradiction, that all four roots of $p(\mu)$ are inside the unit circle. Then $\left|\mu_{1}\right|<1$ by assumption. However (A.14) implies $\left|\beta \mu_{2}\right|>1$, and thus $\left|\mu_{2}\right|>1$, which contradicts the assumption that all four roots are inside the unit circle. 
- Let us suppose next that $p(\mu)$ has three roots inside the unit circle. If $\left|\mu_{1}\right|<1$, then (A.14) implies again $\left|\mu_{2}\right|>1$. It follows that the remaining two roots $\mu_{3}$ and $\mu_{4}$ must be inside the unit circle. But this is impossible, as $\left|\mu_{3}\right|<1$ implies $\left|\mu_{4}\right|>1$. Inversely, if $\left|\mu_{1}\right|>1$, then the three remaining roots must be inside the unit circle. Again, this is impossible as $\left|\mu_{3}\right|<1$ implies $\left|\mu_{4}\right|>1$.

It follows that $p(\mu)$ must have exactly 2 roots inside the unit circle, and thus that the equilibrium is determinate.

\section{A.5 Proposition 7}

Proposition 7. Assuming $0<\lambda_{1}<1<\lambda_{2}$, two bounded stochastic processes $\left\{\hat{\imath}_{t}, \hat{q}_{t}\right\}$ satisfy

$$
\left(1-\lambda_{1} L\right)\left(1-\lambda_{2} L\right) \hat{\imath}_{t}=\hat{q}_{t}
$$

for all $t \geq 0$ if and only if they satisfy

$$
\left(1-\lambda_{1} L\right) \hat{\imath}_{t-1}=-\lambda_{2}^{-1} \mathrm{E}_{t}\left[\left(1-\lambda_{2}^{-1} L^{-1}\right)^{-1} \hat{q}_{t}\right]
$$

for all $t \geq 0$.

Proof: First, we show that (A.15) implies (A.16). Expanding the left-hand side of (A.15), we obtain

$$
\left(1-\lambda_{1} L\right) \hat{\imath}_{t}-\lambda_{2}\left(1-\lambda_{1} L\right) \hat{\imath}_{t-1}=\hat{q}_{t},
$$

or equivalently

$$
\begin{aligned}
\left(1-\lambda_{1} L\right) \hat{\imath}_{t-1} & =-\lambda_{2}^{-1}\left[\hat{q}_{t}-\left(1-\lambda_{1} L\right) \hat{\imath}_{t}\right] \\
& =-\lambda_{2}^{-1} \mathrm{E}_{t}\left[\hat{q}_{t}-\left(1-\lambda_{1} L\right) \hat{\imath}_{t}\right] .
\end{aligned}
$$

Substituting recursively for $\left(1-\lambda_{1} L\right) \hat{t}_{t+j}$ on the right hand side, we obtain

$$
\begin{aligned}
\left(1-\lambda_{1} L\right) \hat{\imath}_{t-1} & =-\lambda_{2}^{-1} \mathrm{E}_{t}\left[\sum_{j=0}^{\infty} \lambda_{2}^{-j} \hat{q}_{t+j}\right] \\
& =-\lambda_{2}^{-1} \mathrm{E}_{t}\left[\left(1-\lambda_{2}^{-1} L^{-1}\right)^{-1} \hat{q}_{t}\right]
\end{aligned}
$$


where the last equality holds since $0<\lambda_{2}^{-1}<1$. Thus (A.15) implies (A.16).

Second, we show that (A.16) implies (A.15). Since (A.16) holds for all $t \geq 0$, it implies that

$$
\left(1-\lambda_{1} L\right) \hat{\imath}_{t}=-\lambda_{2}^{-1} \mathrm{E}_{t+1}\left[\left(1-\lambda_{2}^{-1} L^{-1}\right)^{-1} \hat{q}_{t+1}\right] .
$$

Multiplying both sides by $\left(1-\lambda_{2} L\right)$ and taking conditional expectations at date $t$, we obtain

$$
\begin{aligned}
\left(1-\lambda_{1} L\right)\left(1-\lambda_{2} L\right) \hat{\imath}_{t} & =\mathrm{E}_{t}\left[\left(1-\lambda_{2}^{-1} L^{-1}\right)^{-1}\left(L-\lambda_{2}^{-1}\right) \hat{q}_{t+1}\right] \\
& =\mathrm{E}_{t}\left[\left(1-\lambda_{2}^{-1} L^{-1}\right)^{-1}\left(1-\lambda_{2}^{-1} L^{-1}\right) L \hat{q}_{t+1}\right] \\
& =\mathrm{E}_{t}\left[L \hat{q}_{t+1}\right] \\
& =\hat{q}_{t} .
\end{aligned}
$$

Thus (A.16) also implies (A.15).

\section{A.6 Proposition 8}

Proposition 8. Suppose that $\sigma, \kappa>0,0<\beta<1$, and $0<\gamma \leq 1$. Then in the factorization

$$
A(L)=\beta \gamma\left(1-\lambda_{1} L\right)\left(1-\lambda_{2} L\right)\left(1-\lambda_{3} L\right)
$$

of the polynomial

$$
A(L) \equiv \beta \gamma-(1+\gamma+\beta \gamma) L+\left(1+\gamma+\beta^{-1}(1+\kappa \sigma)\right) L^{2}-\beta^{-1} L^{3}
$$

there is necessarily one real root $0<\lambda_{1}<1$, and two roots outside the unit circle. The latter two roots are either two real roots $\lambda_{3} \geq \lambda_{2}>1$, or a complex pair $\lambda_{2}, \lambda_{3}$ with real part greater than 1 . Three real roots necessarily exist for all small enough $\gamma>0$, while a complex pair necessarily exists for all $\gamma$ close enough to 1 .

Proof: Consider the following properties of the polynomial (A.18):

$$
\begin{array}{ll}
A(z)>0, \forall z \leq 0 & A^{\prime}(z)<0, \forall z \leq 0 \\
A(\beta)=\beta \kappa \sigma>0 & A^{\prime}(\beta)=(1-\beta)(1-\gamma)+2 \kappa \sigma>0 \\
A(1)=\beta^{-1} \kappa \sigma>0 & \\
A(+\infty)=-\infty &
\end{array}
$$


From this, we know that for all $z \leq 0, A(z)$ is positive and decreasing. As $z$ is raised from 0 to $\beta, A(z)$ continues to decrease, reaches a minimum (where $A(z)$ may be positive or negative), and starts increasing as $z$ approaches $\beta$. The polynomial $A(z)$ is positive for $z=1$, but decreases again and tends to $-\infty$, as $z$ becomes larger and larger. It follows that $A(z)$, admits one real root $z_{1}>1$ and either two real roots $0<z_{3} \leq z_{2}<1$, or a pair of complex roots $z_{2}, z_{3}$.

Thus $A(L)$ can be written as

$$
\begin{aligned}
A(L) & =\beta^{-1}\left(z_{1}-L\right)\left(z_{2}-L\right)\left(z_{3}-L\right) \\
& =\beta^{-1} \lambda_{1}^{-1} \lambda_{2}^{-1} \lambda_{3}^{-1}\left(1-\lambda_{1} L\right)\left(1-\lambda_{2} L\right)\left(1-\lambda_{3} L\right) \\
& =\frac{1}{\beta \lambda_{1} \lambda_{2} \lambda_{3}}-\frac{\lambda_{1}+\lambda_{2}+\lambda_{3}}{\beta \lambda_{1} \lambda_{2} \lambda_{3}} L+\frac{\lambda_{1} \lambda_{2}+\left(\lambda_{1}+\lambda_{2}\right) \lambda_{3}}{\beta \lambda_{1} \lambda_{2} \lambda_{3}} L^{2}-\beta^{-1} L^{3}
\end{aligned}
$$

where $\lambda_{j} \equiv z_{j}^{-1}$ for $j=1,2,3$. Comparing the first terms of (A.19) and (A.18), we note that

$$
\left(\beta \lambda_{1} \lambda_{2} \lambda_{3}\right)^{-1}=\beta \gamma
$$

so that the polynomial $A(L)$ can be factorized as in (A.17), where $0<\lambda_{1}<1$ and $\lambda_{2}, \lambda_{3}$ are either two real roots satisfying $1<\lambda_{2} \leq \lambda_{3}$, or a pair of complex roots.

We now show that in the case that $\lambda_{2}, \lambda_{3}$ form a pair of complex roots, their common real part is greater than 1. Comparing the second term of (A.19) with the corresponding term in (A.18), and using (A.20), we note that

$$
\beta \gamma\left(\lambda_{1}+\lambda_{2}+\lambda_{3}\right)=1+\gamma+\beta \gamma .
$$

Furthermore, as $\beta \gamma \lambda_{1}<1$, we have

$$
\beta \gamma \lambda_{1}=1+\gamma-\beta \gamma\left(\lambda_{2}+\lambda_{3}-1\right)<1 .
$$

This implies

$$
-\beta \gamma\left(\lambda_{2}+\lambda_{3}-1\right)<-\gamma,
$$

and thus

$$
\lambda_{2}+\lambda_{3}>1+\beta^{-1}>2 .
$$


Therefore

$$
\operatorname{Re} \lambda_{2}=\operatorname{Re} \lambda_{3}=\frac{\lambda_{2}+\lambda_{3}}{2}>1 .
$$

It follows that the moduli $\left|\lambda_{2}\right|=\left|\lambda_{3}\right|>1$.

We now show that three real roots $\lambda_{1}, \lambda_{2}, \lambda_{3}$ necessarily exist for all small enough $\gamma>0$, while a complex pair $\lambda_{2}, \lambda_{3}$ necessarily exists for all $\gamma$ close enough to 1 . First note that each $\lambda_{j}$, for $j=1,2,3$, is real if and only if the solution $z_{j} \equiv \lambda_{j}^{-1}$ of the equation

$$
A(z) \equiv A_{0}+A_{1} z+A_{2} z^{2}+A_{3} z^{3}=0
$$

is real, where

$$
\begin{aligned}
& A_{0}=\beta \gamma \\
& A_{1}=-(1+\gamma+\beta \gamma) \\
& A_{2}=1+\gamma+\beta^{-1}(1+\kappa \sigma) \\
& A_{3}=-\beta^{-1} .
\end{aligned}
$$

Furthermore, since

$$
A(z)=A_{3}\left(B_{0}+B_{1} \zeta+\zeta^{3}\right)
$$

where

$$
\zeta=z+\frac{A_{2}}{3 A_{3}}
$$

and

$$
\begin{aligned}
B_{0} & =\frac{27 A_{0} A_{3}^{2}+2 A_{2}^{3}-9 A_{3} A_{2} A_{1}}{27 A_{3}^{3}} \\
B_{1} & =\frac{3 A_{3} A_{1}-A_{2}^{2}}{3 A_{3}^{2}}
\end{aligned}
$$

are real coefficients, each $\lambda_{j}$ is real if and only if the corresponding solution $\zeta_{j}$ of the equation

$$
B_{0}+B_{1} \zeta+\zeta^{3}=0
$$

is real (see Lemma 1 below). From Cardano's formulas for the roots of a cubic equation, we know that this equation admits: 
- three different real roots if $\Delta \equiv 27 B_{0}^{2}+4 B_{1}^{3}<0$,

- three real roots, at least two of which are equal, if $\Delta=0$,

- one real root and two complex roots if $\Delta>0$.

Expressing $\Delta$ as a function of $\gamma$, we have:

$$
\begin{aligned}
\Delta(\gamma)= & 27 B_{0}^{2}+4 B_{1}^{3}=A_{3}^{-4}\left(27 A_{0}^{2} A_{3}^{2}+4 A_{0} A_{2}^{3}-18 A_{0} A_{3} A_{2} A_{1}-A_{2}^{2} A_{1}^{2}+4 A_{3} A_{1}^{3}\right) \\
= & -\beta^{4}(1-\beta)^{2} \gamma^{4}-2 \beta^{3}\left(\left(1+\beta^{2}-4 \beta\right) \kappa \sigma-(1+\beta)(1-\beta)^{2}\right) \gamma^{3} \\
& -\beta^{2}\left(\kappa^{2} \sigma^{2}\left(1-10 \beta+\beta^{2}\right)+2 \kappa \sigma(1+\beta)\left(1+\beta+\beta^{2}\right)+\left(1+4 \beta+\beta^{2}\right)(1-\beta)^{2}\right) \gamma^{2} \\
& +\beta^{2}\left(4 \kappa^{3} \sigma^{3}+10(1+\beta) \kappa^{2} \sigma^{2}+4\left(2+2 \beta^{2}-\beta\right) \kappa \sigma+2(1+\beta)(1-\beta)^{2}\right) \gamma \\
& -\beta^{2}\left(2 \beta \kappa \sigma+\kappa^{2} \sigma^{2}+2 \kappa \sigma+(1-\beta)^{2}\right)
\end{aligned}
$$

which is a fourth-order polynomial in $\gamma$. Note that $\Delta(\gamma)$ is a continuous function of $\gamma$ that admits at most four real roots, and has the following properties:

$$
\begin{aligned}
& \Delta(-\infty)=-\infty \\
& \Delta(0)=-\beta^{2}\left(2 \beta \kappa \sigma+\kappa^{2} \sigma^{2}+2 \kappa \sigma+(1-\beta)^{2}\right)<0 \\
& \Delta(1)=\beta^{2} \kappa \sigma\left(4 \kappa^{2} \sigma^{2}+\left(8+20 \beta-\beta^{2}\right) \kappa \sigma+4(1-\beta)^{3}\right)>0 \\
& \Delta(+\infty)=-\infty .
\end{aligned}
$$

It follows that $\Delta(\gamma)$ admits either one or three roots between 0 and 1 . Furthermore, $\Delta<0$ for $\gamma>0$ small enough, and $\Delta>0$ for all $\gamma$ close enough to 1 . Thus three real roots necessarily exist for all small enough $\gamma>0$, while a complex pair necessarily exists for all $\gamma$ close enough to 1 .

\section{A.7 Proposition 9}

Proposition 9. Under the assumptions of Proposition 8, and in the case that the factor-

ization (2.9) involves three real roots, a pair of bounded processes $\left\{\hat{\imath}_{t}, \hat{f}_{t}\right\}$ satisfy any of the equations

$$
\begin{aligned}
\left(1-\lambda_{1} L\right)\left(1-\lambda_{2} L\right) \hat{\imath}_{t} & =\left(\beta \gamma \lambda_{3}\right)^{-1} \mathrm{E}_{t}\left[\left(1-\lambda_{3}^{-1} L^{-1}\right)^{-1} \hat{f}_{t}\right] \\
\left(1-\lambda_{1} L\right)\left(1-\lambda_{3} L\right) \hat{\imath}_{t} & =\left(\beta \gamma \lambda_{2}\right)^{-1} \mathrm{E}_{t}\left[\left(1-\lambda_{2}^{-1} L^{-1}\right)^{-1} \hat{f}_{t}\right]
\end{aligned}
$$


or

$$
\begin{aligned}
\left(1-\lambda_{1} L\right)\left(1-\frac{\lambda_{2}+\lambda_{3}}{2} L\right) \hat{\imath}_{t}= & \frac{1}{2}\left(\beta \gamma \lambda_{3}\right)^{-1} \mathrm{E}_{t}\left[\left(1-\lambda_{3}^{-1} L^{-1}\right)^{-1} \hat{f}_{t}\right] \\
& +\frac{1}{2}\left(\beta \gamma \lambda_{2}\right)^{-1} \mathrm{E}_{t}\left[\left(1-\lambda_{2}^{-1} L^{-1}\right)^{-1} \hat{f}_{t}\right]
\end{aligned}
$$

at all dates $t \geq t_{0}$ if and only if they satisfy

$$
\mathrm{E}_{t}\left[A(L) \hat{\imath}_{t+1}\right]=-\hat{f}_{t}
$$

at all of those same dates. In the case that a complex pair exists, (A.24) is again equivalent to (A.25) in the same sense.

Proof: Proposition 8 guarantees that the roots $\lambda_{1}, \lambda_{2}, \lambda_{3}$ in the factorization (2.9) are either real and satisfy $0<\lambda_{1}<1<\lambda_{2} \leq \lambda_{3}$, or $0<\lambda_{1}<1$, and $\lambda_{2}, \lambda_{3}$ are complex conjugates that lie outside the unit circle. Consider first the case in which $\lambda_{1}, \lambda_{2}, \lambda_{3}$ are real.

\section{Rule I.}

We first show that (A.25) implies (A.22). Using (2.9) to substitute for $A(L)$, and expanding the left-hand side of (A.25), we obtain

$$
\beta \gamma \mathrm{E}_{t}\left[\left(1-\lambda_{1} L\right)\left(1-\lambda_{2} L\right) \hat{\imath}_{t+1}-\left(1-\lambda_{1} L\right)\left(1-\lambda_{2} L\right) \lambda_{3} \hat{\imath}_{t}\right]=-\hat{f}_{t},
$$

or

$$
\left(1-\lambda_{1} L\right)\left(1-\lambda_{2} L\right) \hat{\imath}_{t}=\left(\beta \gamma \lambda_{3}\right)^{-1} \hat{f}_{t}+\lambda_{3}^{-1} \mathrm{E}_{t}\left[\left(1-\lambda_{1} L\right)\left(1-\lambda_{2} L\right) \hat{\imath}_{t+1}\right] .
$$

Substituting recursively for $\left(1-\lambda_{1} L\right)\left(1-\lambda_{2} L\right) \hat{\imath}_{t+j}$ on the right hand side, we obtain

$$
\begin{aligned}
\left(1-\lambda_{1} L\right)\left(1-\lambda_{2} L\right) \hat{\imath}_{t} & =\left(\beta \gamma \lambda_{3}\right)^{-1} \mathrm{E}_{t}\left[\sum_{j=0}^{\infty} \lambda_{3}^{-j} \hat{f}_{t+j}\right] \\
& =\left(\beta \gamma \lambda_{3}\right)^{-1} \mathrm{E}_{t}\left[\left(1-\lambda_{3}^{-1} L^{-1}\right)^{-1} \hat{f}_{t}\right]
\end{aligned}
$$

which corresponds to (A.22).

We next show that (A.22) implies (A.25). Since (A.22) holds for all $t \geq t_{0}$, it implies that

$$
\left(1-\lambda_{1} L\right)\left(1-\lambda_{2} L\right) \hat{\imath}_{t+1}=\left(\beta \gamma \lambda_{3}\right)^{-1} \mathrm{E}_{t+1}\left[\left(1-\lambda_{3}^{-1} L^{-1}\right)^{-1} \hat{f}_{t+1}\right]
$$


Multiplying both sides by $\beta \gamma\left(1-\lambda_{3} L\right)$, taking conditional expectations at date $t$, and using (2.9), we obtain

$$
\begin{aligned}
\mathrm{E}_{t}\left[A(L) \hat{\imath}_{t+1}\right] & =\lambda_{3}^{-1} \mathrm{E}_{t}\left[\left(1-\lambda_{3} L\right)\left(1-\lambda_{3}^{-1} L^{-1}\right)^{-1} \hat{f}_{t+1}\right] \\
& =\mathrm{E}_{t}\left[\left(1-\lambda_{3}^{-1} L^{-1}\right)\left(1-\lambda_{3}^{-1} L^{-1}\right)^{-1} L \hat{f}_{t+1}\right] \\
& =-\mathrm{E}_{t}\left[L \hat{f}_{t+1}\right] \\
& =-\hat{f}_{t} .
\end{aligned}
$$

which corresponds to (A.25).

\section{Rule II.}

To show that a pair of bounded processes $\left\{\hat{\imath}_{t}, \hat{f}_{t}\right\}$ satisfy (A.23) at all dates if and only if they satisfy (A.25) at all dates, we simply need to repeat the above steps, replacing $\lambda_{2}$ with $\lambda_{3}$ and vice versa.

\section{Rule III.}

Let us now allow $\lambda_{2}, \lambda_{3}$ to be either real values, or complex conjugates, lying outside the unit circle. Since (A.25) implies both (A.22) and (A.23), we know that (A.25) implies

$$
\begin{aligned}
\left(1-\lambda_{1} L\right)\left[\frac{\left(1-\lambda_{2} L\right)+\left(1-\lambda_{3} L\right)}{2}\right] \hat{\imath}_{t}= & \frac{1}{2}\left(\beta \gamma \lambda_{3}\right)^{-1} \mathrm{E}_{t}\left[\left(1-\lambda_{3}^{-1} L^{-1}\right)^{-1} \hat{f}_{t}\right] \\
& +\frac{1}{2}\left(\beta \gamma \lambda_{2}\right)^{-1} \mathrm{E}_{t}\left[\left(1-\lambda_{2}^{-1} L^{-1}\right)^{-1} \hat{f}_{t}\right],
\end{aligned}
$$

which is obtained by summing (A.22) and (A.23) on both sides and dividing by 2 . Thus (A.25) implies (A.24).

We next show that (A.24) implies (A.25). Since (A.24) holds for all $t \geq t_{0}$, it implies that

$$
\begin{aligned}
\left(1-\lambda_{1} L\right)\left(1-\frac{\lambda_{2}+\lambda_{3}}{2} L\right) \hat{\imath}_{t+2}= & \left(2 \beta \gamma \lambda_{3}\right)^{-1} \mathrm{E}_{t+2}\left[\left(1-\lambda_{3}^{-1} L^{-1}\right)^{-1} \hat{f}_{t+2}\right] \\
& +\left(2 \beta \gamma \lambda_{2}\right)^{-1} \mathrm{E}_{t+2}\left[\left(1-\lambda_{2}^{-1} L^{-1}\right)^{-1} \hat{f}_{t+2}\right] .
\end{aligned}
$$

Multiplying both sides by $\beta \gamma\left(1-\lambda_{2} L\right)\left(1-\lambda_{3} L\right)$, taking conditional expectations at date 
$t$, and using (2.9), we obtain

$$
\begin{aligned}
\mathrm{E}_{t}\left[A(L)\left(1-\frac{\lambda_{2}+\lambda_{3}}{2} L\right) \hat{\imath}_{t+2}\right]= & \frac{1}{2 \lambda_{3}} \mathrm{E}_{t}\left[\left(1-\lambda_{2} L\right)\left(1-\lambda_{3} L\right)\left(1-\lambda_{3}^{-1} L^{-1}\right)^{-1} \hat{f}_{t+2}\right] \\
& +\frac{1}{2 \lambda_{2}} \mathrm{E}_{t}\left[\left(1-\lambda_{2} L\right)\left(1-\lambda_{3} L\right)\left(1-\lambda_{2}^{-1} L^{-1}\right)^{-1} \hat{f}_{t+2}\right] \\
= & -\frac{1}{2} \mathrm{E}_{t}\left[\left(1-\lambda_{2} L\right) \hat{f}_{t+1}\right]-\frac{1}{2} \mathrm{E}_{t}\left[\left(1-\lambda_{3} L\right) \hat{f}_{t+1}\right] \\
= & -\mathrm{E}_{t}\left[\left(1-\frac{\lambda_{2}+\lambda_{3}}{2} L\right) \hat{f}_{t+1}\right] .
\end{aligned}
$$

It follows that

$$
-\mathrm{E}_{t}\left[A(L)\left(1-\frac{2}{\lambda_{2}+\lambda_{3}} L^{-1}\right) L \hat{\imath}_{t+2}\right]=\mathrm{E}_{t}\left[\left(1-\frac{2}{\lambda_{2}+\lambda_{3}} L^{-1}\right) L \hat{f}_{t+1}\right]
$$

and hence that

$$
\mathrm{E}_{t}\left[A(L)\left(1-\alpha L^{-1}\right) \hat{\imath}_{t+1}\right]=v_{t}
$$

where $0 \leq \alpha \equiv \frac{2}{\lambda_{2}+\lambda_{3}}<1$, and $v_{t} \equiv-\mathrm{E}_{t}\left[\left(1-\alpha L^{-1}\right) \hat{f}_{t}\right]$. This implies furthermore

$$
\begin{aligned}
\mathrm{E}_{t}\left[A(L) \hat{\imath}_{t+1}\right] & =\alpha \mathrm{E}_{t}\left[A(L) \hat{\imath}_{t+2}\right]+v_{t} \\
& =\mathrm{E}_{t}\left[\sum_{j=0}^{\infty} \alpha^{j} v_{t+j}\right] \\
& =\mathrm{E}_{t}\left[\left(1-\alpha L^{-1}\right)^{-1} v_{t}\right] \\
& =-\hat{f}_{t},
\end{aligned}
$$

which corresponds to (A.25).

\section{A.8 Proposition 10}

Proposition 10. Under the assumptions of Proposition 8, and a loss function with $\lambda_{x}, \lambda_{i}>$ 0 , each of the rules I, II, and III has a representation of the form

$$
i_{t}=\left(1-\rho_{1}\right) i^{*}+\rho_{1} i_{t-1}+\rho_{2} \Delta i_{t-1}+\phi_{\pi} F_{t}(\pi)+\frac{\phi_{x}}{4} F_{t}(x)-\theta_{\pi} \pi_{t-1}-\frac{\theta_{x}}{4} x_{t-1}
$$


for all values of $\gamma$ for which the rule is well-defined, and in this representation

$$
\begin{aligned}
\rho_{1} & >1, \quad \rho_{2}>0 \\
0 & <\theta_{\pi} \leq \phi_{\pi} \\
0 & <\theta_{x}=\phi_{x} .
\end{aligned}
$$

Furthermore, for given values of the other parameters, as $\gamma \rightarrow 0$ (for rule I) the coefficient $\theta_{\pi}$ approaches zero, though $\phi_{\pi}$ approaches a positive limit; while as $\gamma \rightarrow 1$ (for rule III) the coefficients $\theta_{\pi}$ and $\phi_{\pi}$ approach the same limit.

ProOF: Proposition 8 guarantees that the roots $\lambda_{1}, \lambda_{2}, \lambda_{3}$ in the factorization (2.9) are either real and satisfy $0<\lambda_{1}<1<\lambda_{2} \leq \lambda_{3}$, or $0<\lambda_{1}<1$, and $\lambda_{2}, \lambda_{3}$ are complex conjugates that lie outside the unit circle. Consider first the case in which $\lambda_{1}, \lambda_{2}, \lambda_{3}$ are real, so that both rule I and rule II are well-defined.

\section{Rule I:}

First note that the rule I, i.e., (2.11) can be rewritten as

$$
\hat{\imath}_{t}=\rho_{1} \hat{\imath}_{t-1}+\rho_{2} \Delta \hat{\imath}_{t-1}+\left(\beta \gamma \lambda_{3}\right)^{-1} v_{t}
$$

where

$$
\begin{aligned}
& \rho_{1}=1+\left(\lambda_{2}-1\right)\left(1-\lambda_{1}\right)>1 \\
& \rho_{2}=\lambda_{1} \lambda_{2}>0
\end{aligned}
$$

and

$$
\begin{aligned}
v_{t} & \equiv \mathrm{E}_{t}\left[\left(1-\lambda_{3}^{-1} L^{-1}\right)^{-1} \hat{f}_{t}\right] \\
& =\mathrm{E}_{t}\left[\sum_{j=0}^{\infty} \lambda_{3}^{-j} \hat{f}_{t+j}\right] .
\end{aligned}
$$


Since $\mathrm{E}_{t} \hat{f}_{t+j}$ is given by

$$
\begin{aligned}
\mathrm{E}_{t} \hat{f}_{t+j}= & \frac{\kappa \sigma}{\lambda_{i}} \mathrm{E}_{t}\left(\hat{q}_{t+j}-\beta \gamma \hat{q}_{t+j+1}\right) \\
= & \frac{\kappa \sigma}{\lambda_{i}} \mathrm{E}_{t}\left[-\gamma \hat{\pi}_{t+j-1}+\left(1+\beta \gamma^{2}\right) \hat{\pi}_{t+j}-\beta \gamma \hat{\pi}_{t+j+1}\right] \\
& +\frac{\lambda_{x} \sigma}{\lambda_{i}} \mathrm{E}_{t}\left[-\hat{x}_{t+j-1}+(1+\beta \gamma) \hat{x}_{t+j}-\beta \gamma \hat{x}_{t+j+1}\right]
\end{aligned}
$$

we have

$$
\begin{aligned}
v_{t}= & \frac{\kappa \sigma}{\lambda_{i}} \mathrm{E}_{t}\left[\sum_{j=0}^{\infty} \lambda_{3}^{-j}\left(-\gamma \hat{\pi}_{t+j-1}+\left(1+\beta \gamma^{2}\right) \hat{\pi}_{t+j}-\beta \gamma \hat{\pi}_{t+j+1}\right)\right] \\
& +\frac{\lambda_{x} \sigma}{\lambda_{i}} \mathrm{E}_{t}\left[\sum_{j=0}^{\infty} \lambda_{3}^{-j}\left(-\hat{x}_{t+j-1}+(1+\beta \gamma) \hat{x}_{t+j}-\beta \gamma \hat{x}_{t+j+1}\right)\right] \\
= & \frac{\kappa \sigma}{\lambda_{i}} \sum_{j=-1}^{\infty} \tilde{\alpha}_{\pi, j} \mathrm{E}_{t} \hat{\pi}_{t+j}+\frac{\lambda_{x} \sigma}{\lambda_{i}} \sum_{j=-1}^{\infty} \alpha_{x, j} \mathrm{E}_{t} \hat{x}_{t+j},
\end{aligned}
$$

where

$$
\begin{aligned}
\tilde{\alpha}_{\pi,-1} & =-\gamma \\
\tilde{\alpha}_{\pi, 0} & =1+\beta \gamma^{2}-\lambda_{3}^{-1} \gamma \\
\tilde{\alpha}_{\pi, j} & =-\lambda_{3}^{-j+1} \beta \gamma+\lambda_{3}^{-j}\left(1+\beta \gamma^{2}\right)-\lambda_{3}^{-j-1} \gamma, \quad \forall j \geq 1
\end{aligned}
$$

and

$$
\begin{aligned}
\alpha_{x,-1} & =-1 \\
\alpha_{x, 0} & =1+\beta \gamma-\lambda_{3}^{-1} \\
\alpha_{x, j} & =-\lambda_{3}^{-j+1} \beta \gamma+\lambda_{3}^{-j}(1+\beta \gamma)-\lambda_{3}^{-j-1}, \quad \forall j \geq 1 .
\end{aligned}
$$

The variable $v_{t}$ can furthermore be written as

$$
v_{t}=\frac{\kappa \sigma}{\lambda_{i}} S_{\pi} \sum_{j=0}^{\infty} \alpha_{\pi, j} \mathrm{E}_{t} \hat{\pi}_{t+j}+\frac{\lambda_{x} \sigma}{\lambda_{i}} \sum_{j=0}^{\infty} \alpha_{x, j} \mathrm{E}_{t} \hat{x}_{t+j}-\frac{\kappa \sigma \gamma}{\lambda_{i}} \hat{\pi}_{t-1}-\frac{\lambda_{x} \sigma}{\lambda_{i}} \hat{x}_{t-1}
$$


where

$$
\begin{aligned}
S_{\pi}= & \sum_{j=0}^{\infty} \tilde{\alpha}_{\pi, j} \\
= & -\left(0+\lambda_{3}^{-0}+\lambda_{3}^{-1}+\lambda_{3}^{-2}+\ldots\right) \beta \gamma+\left(\lambda_{3}^{-0}+\lambda_{3}^{-1}+\lambda_{3}^{-2}+\ldots\right)\left(1+\beta \gamma^{2}\right) \\
& -\left(\lambda_{3}^{-1}+\lambda_{3}^{-2}+\ldots\right) \gamma \\
= & \left(1-\lambda_{3}^{-1}\right)^{-1}\left(1+\beta \gamma^{2}-\beta \gamma-\lambda_{3}^{-1} \gamma\right),
\end{aligned}
$$

and

$$
\alpha_{\pi, j}=\frac{\tilde{\alpha}_{\pi, j}}{S_{\pi}}, \quad \forall j \geq 0 .
$$

Note that the coefficients $\alpha_{\pi, j}$ satisfy

$$
\sum_{j=0}^{\infty} \alpha_{\pi, j}=S_{\pi}^{-1} \sum_{j=0}^{\infty} \tilde{\alpha}_{\pi, j}=1
$$

and the coefficients $\alpha_{x, j}$ satisfy

$$
\begin{aligned}
\sum_{j=0}^{\infty} \alpha_{x, j}= & -\left(0+\lambda_{3}^{-0}+\lambda_{3}^{-1}+\lambda_{3}^{-2}+\ldots\right) \beta \gamma+\left(\lambda_{3}^{-0}+\lambda_{3}^{-1}+\lambda_{3}^{-2}+\ldots\right)(1+\beta \gamma) \\
& -\left(\lambda_{3}^{-1}+\lambda_{3}^{-2}+\ldots\right) \\
= & \left(1-\lambda_{3}^{-1}\right)^{-1}\left(1-\lambda_{3}^{-1}\right) \\
= & 1 .
\end{aligned}
$$

Combining (A.27) and (A.34), we can rewrite the rule I as

$$
\hat{\imath}_{t}=\rho_{1} \hat{\imath}_{t-1}+\rho_{2} \Delta \hat{\imath}_{t-1}+\phi_{\pi} F_{t}(\hat{\pi})+\frac{\phi_{x}}{4} F_{t}(\hat{x})-\theta_{\pi} \hat{\pi}_{t-1}-\frac{\theta_{x}}{4} \hat{x}_{t-1}
$$

where

$$
\begin{aligned}
\phi_{\pi} & =\left(\beta \gamma \lambda_{3}\right)^{-1} \frac{\kappa \sigma}{\lambda_{i}} S_{\pi}=\frac{\kappa \sigma}{\lambda_{i} \beta} \frac{1+\beta \gamma^{2}-\beta \gamma-\lambda_{3}^{-1} \gamma}{\lambda_{3} \gamma\left(1-\lambda_{3}^{-1}\right)} \\
\theta_{\pi} & =\left(\beta \gamma \lambda_{3}\right)^{-1} \frac{\kappa \sigma \gamma}{\lambda_{i}}=\frac{\kappa \sigma}{\lambda_{i} \beta \lambda_{3}}>0 \\
\phi_{x} & =\theta_{x}=\frac{4 \lambda_{x} \sigma}{\lambda_{i} \beta \gamma \lambda_{3}}>0 .
\end{aligned}
$$


Note furthermore that

$$
\phi_{\pi}=\theta_{\pi} \frac{\lambda_{3}+\beta \gamma^{2} \lambda_{3}-\beta \gamma \lambda_{3}-\gamma}{\gamma\left(\lambda_{3}-1\right)}=\theta_{\pi}\left(1+\frac{(1-\gamma)(1-\beta \gamma)}{\gamma\left(1-\lambda_{3}^{-1}\right)}\right) \geq \theta_{\pi} .
$$

Recalling that $\hat{z}_{t} \equiv z_{t}-\bar{z}$ for any variable $z$, and that $\phi_{x}=\theta_{x}$, we can rewrite (A.35) as

$$
i_{t}=\left(1-\rho_{1}\right) \bar{\imath}-\left(\phi_{\pi}-\theta_{\pi}\right) \bar{\pi}+\rho_{1} i_{t-1}+\rho_{2} \Delta i_{t-1}+\phi_{\pi} F_{t}(\pi)+\frac{\phi_{x}}{4} F_{t}(x)-\theta_{\pi} \pi_{t-1}-\frac{\theta_{x}}{4} x_{t-1} .
$$

We know from Proposition 9 that (2.10) holds, and thus that (2.5) holds. In the steady state, equation (2.5) reduces to

$$
A(L)\left(\bar{\imath}-i^{*}\right)=-\bar{f}
$$

where

$$
\bar{f}=\frac{\kappa \sigma}{\lambda_{i}}(1-\beta \gamma) \bar{q}=\frac{\kappa \sigma}{\lambda_{i}}(1-\beta \gamma)(1-\gamma) \bar{\pi} .
$$

It follows from (2.9) that

$$
\left(1-\lambda_{1}\right)\left(1-\lambda_{2}\right) i^{*}=\left(1-\lambda_{1}\right)\left(1-\lambda_{2}\right) \bar{\imath}+\frac{\kappa \sigma}{\lambda_{i}} \frac{(1-\gamma)(1-\beta \gamma)}{\beta \gamma\left(1-\lambda_{3}\right)} \bar{\pi} .
$$

Given that

$$
\left(1-\lambda_{1}\right)\left(1-\lambda_{2}\right)=1-\rho_{1}
$$

and given that (A.36) implies

$$
\frac{\kappa \sigma}{\lambda_{i}} \frac{(1-\gamma)(1-\beta \gamma)}{\beta \gamma\left(1-\lambda_{3}\right)}=-\left(\phi_{\pi}-\theta_{\pi}\right)
$$

we can rewrite (A.38) as

$$
\left(1-\rho_{1}\right) i^{*}=\left(1-\rho_{1}\right) \bar{\imath}-\left(\phi_{\pi}-\theta_{\pi}\right) \bar{\pi}
$$

Combining this with (A.37) yields (A.26).

As $\gamma$ approaches 0 , we have $\lambda_{3}^{-1} \rightarrow 0$ and $\lambda_{3} \gamma \rightarrow \beta^{-1}$. It follows that

$$
\begin{aligned}
& \lim _{\gamma \rightarrow 0} \phi_{\pi}=\lim _{\gamma \rightarrow 0} \frac{\kappa \sigma}{\lambda_{i} \beta} \frac{1+\beta \gamma^{2}-\beta \gamma-\lambda_{3}^{-1} \gamma}{\lambda_{3} \gamma\left(1-\lambda_{3}^{-1}\right)}=\frac{\kappa \sigma}{\lambda_{i}}>0 \\
& \lim _{\gamma \rightarrow 0} \theta_{\pi}=\lim _{\gamma \rightarrow 0} \frac{\kappa \sigma}{\lambda_{i} \beta} \lambda_{3}^{-1}=0 \\
& \lim _{\gamma \rightarrow 0} \phi_{x}=\lim _{\gamma \rightarrow 0} \theta_{x}=\lim _{\gamma \rightarrow 0} \frac{4 \lambda_{x} \sigma}{\lambda_{i} \beta \gamma \lambda_{3}}=\frac{4 \lambda_{x} \sigma}{\lambda_{i}}>0 .
\end{aligned}
$$




\section{Rule II}

Following the same development as for rule I, but replacing $\lambda_{2}$ with $\lambda_{3}$ and vice versa, we can show that (2.12) can also be written as in (A.26), but where

$$
\begin{aligned}
\rho_{1} & =1+\left(\lambda_{3}-1\right)\left(1-\lambda_{1}\right)>1 \\
\rho_{2} & =\lambda_{1} \lambda_{3}>0 \\
\theta_{\pi} & =\frac{\kappa \sigma}{\lambda_{i} \beta \lambda_{2}}>0 \\
\phi_{\pi} & =\theta_{\pi}\left(1+\frac{(1-\gamma)(1-\beta \gamma)}{\gamma\left(1-\lambda_{2}^{-1}\right)}\right) \geq \theta_{\pi} \\
\phi_{x} & =\theta_{x}=\frac{4 \lambda_{x} \sigma}{\lambda_{i} \beta \gamma \lambda_{2}}>0 .
\end{aligned}
$$

\section{Rule III}

We now allow the roots $\lambda_{2}$ and $\lambda_{3}$ to be either real or complex. Recall from the proof of Proposition 8 that $\left(\lambda_{2}+\lambda_{3}\right) / 2$ is real and is greater than 1 . Using this, rule III (2.13) can be rewritten, for all values of $\gamma \in(0,1]$, as

$$
\hat{\imath}_{t}=\rho_{1} \hat{\imath}_{t-1}+\rho_{2} \Delta \hat{\imath}_{t-1}+\frac{1}{2}\left(\beta \gamma \lambda_{3}\right)^{-1} v_{t}^{I}+\frac{1}{2}\left(\beta \gamma \lambda_{2}\right)^{-1} v_{t}^{I I}
$$

where $\rho_{1}$ and $\rho_{2}$ are now given by

$$
\begin{aligned}
& \rho_{1}=\lambda_{1}+\frac{\lambda_{2}+\lambda_{3}}{2}-\lambda_{1} \frac{\lambda_{2}+\lambda_{3}}{2}=1+\left(\frac{\lambda_{2}+\lambda_{3}}{2}-1\right)\left(1-\lambda_{1}\right)>1 \\
& \rho_{2}=\lambda_{1} \frac{\lambda_{2}+\lambda_{3}}{2}>0
\end{aligned}
$$

and

$$
\begin{gathered}
v_{t}^{I} \equiv \mathrm{E}_{t}\left[\left(1-\lambda_{3}^{-1} L^{-1}\right)^{-1} \hat{f}_{t}\right]=\frac{\kappa \sigma}{\lambda_{i}} \sum_{j=-1}^{\infty} \tilde{\alpha}_{\pi, j}^{I} \mathrm{E}_{t} \hat{\pi}_{t+j}+\frac{\lambda_{x} \sigma}{\lambda_{i}} \sum_{j=-1}^{\infty} \alpha_{x, j}^{I} \mathrm{E}_{t} \hat{x}_{t+j} \\
v_{t}^{I I} \equiv \mathrm{E}_{t}\left[\left(1-\lambda_{2}^{-1} L^{-1}\right)^{-1} \hat{f}_{t}\right]=\frac{\kappa \sigma}{\lambda_{i}} \sum_{j=-1}^{\infty} \tilde{\alpha}_{\pi, j}^{I I} \mathrm{E}_{t} \hat{\pi}_{t+j}+\frac{\lambda_{x} \sigma}{\lambda_{i}} \sum_{j=-1}^{\infty} \alpha_{x, j}^{I I} \mathrm{E}_{t} \hat{x}_{t+j},
\end{gathered}
$$

and where $\tilde{\alpha}_{\pi, j}^{I}, \alpha_{x, j}^{I}$ are defined in (A.28) - (A.33) for all $j \geq 1, \tilde{\alpha}_{\pi, j}^{I I}, \alpha_{x, j}^{I I}$ are defined in the same way except that $\lambda_{3}$ is replaced with $\lambda_{2}$. Using (A.42) and (A.43), equation (A.39) can 
furthermore be written as

$$
\begin{aligned}
\hat{\imath}_{t}= & \rho_{1} \hat{\imath}_{t-1}+\rho_{2} \Delta \hat{\imath}_{t-1}+\frac{\kappa \sigma}{\lambda_{i} \beta \gamma} \frac{S_{\pi}}{2} \sum_{j=0}^{\infty} \alpha_{\pi, j} \mathrm{E}_{t} \hat{\pi}_{t+j}+\frac{\lambda_{x} \sigma}{\lambda_{i} \beta \gamma} \frac{S_{x}}{2} \sum_{j=0}^{\infty} \alpha_{x, j} \mathrm{E}_{t} \hat{x}_{t+j} \\
& -\frac{\kappa \sigma}{\lambda_{i} \beta} \frac{\lambda_{2}^{-1}+\lambda_{3}^{-1}}{2} \hat{\pi}_{t-1}-\frac{\lambda_{x} \sigma}{\lambda_{i} \beta \gamma} \frac{\lambda_{2}^{-1}+\lambda_{3}^{-1}}{2} \hat{x}_{t-1}
\end{aligned}
$$

where

$$
\begin{aligned}
S_{\pi} & =\sum_{j=0}^{\infty}\left(\lambda_{3}^{-1} \tilde{\alpha}_{\pi, j}^{I}+\lambda_{2}^{-1} \tilde{\alpha}_{\pi, j}^{I I}\right) \\
S_{x} & =\sum_{j=0}^{\infty}\left(\lambda_{3}^{-1} \alpha_{x, j}^{I}+\lambda_{2}^{-1} \alpha_{x, j}^{I I}\right)=\lambda_{2}^{-1}+\lambda_{3}^{-1} \\
\alpha_{\pi, j} & =S_{\pi}^{-1}\left(\lambda_{3}^{-1} \tilde{\alpha}_{\pi, j}^{I}+\lambda_{2}^{-1} \tilde{\alpha}_{\pi, j}^{I I}\right) \\
\alpha_{x, j} & =S_{x}^{-1}\left(\lambda_{3}^{-1} \alpha_{x, j}^{I}+\lambda_{2}^{-1} \alpha_{x, j}^{I I}\right),
\end{aligned}
$$

and where

$$
\sum_{j=0}^{\infty} \alpha_{\pi, j}=\sum_{j=0}^{\infty} \alpha_{x, j}=1
$$

Equation (A.44) is of the form (A.35), where $\rho_{1}$ and $\rho_{2}$ are defined in (A.40), (A.41), and

$$
\begin{aligned}
\phi_{\pi} & =\frac{\kappa \sigma}{\lambda_{i} \beta \gamma} \frac{S_{\pi}}{2} \\
\theta_{\pi} & =\frac{\kappa \sigma}{\lambda_{i} \beta} \frac{\lambda_{2}^{-1}+\lambda_{3}^{-1}}{2}>0 \\
\phi_{x} & =\theta_{x}=\frac{4 \lambda_{x} \sigma}{\lambda_{i} \beta \gamma} \frac{\lambda_{2}^{-1}+\lambda_{3}^{-1}}{2}>0 .
\end{aligned}
$$

(Note that if $\lambda_{2}$ and $\lambda_{3}$ are complex conjugates, then $\lambda_{2}^{-1}+\lambda_{3}^{-1}=\frac{\lambda_{2}+\lambda_{3}}{\lambda_{2} \lambda_{3}}$ is real.) Note furthermore that for all $\gamma \in(0,1]$, the coefficient $\phi_{\pi}$ satisfies

$$
\begin{aligned}
\phi_{\pi} & =\frac{\kappa \sigma}{\lambda_{i} \beta}\left(\frac{\lambda_{3}^{-1}}{2} \frac{1+\beta \gamma^{2}-\beta \gamma-\lambda_{3}^{-1} \gamma}{\gamma\left(1-\lambda_{3}^{-1}\right)}+\frac{\lambda_{2}^{-1}}{2} \frac{1+\beta \gamma^{2}-\beta \gamma-\lambda_{2}^{-1} \gamma}{\gamma\left(1-\lambda_{2}^{-1}\right)}\right) \\
& =\frac{\kappa \sigma}{\lambda_{i} \beta}\left(\frac{\lambda_{3}^{-1}}{2}\left(1+\frac{(1-\gamma)(1-\beta \gamma)}{\gamma\left(1-\lambda_{3}^{-1}\right)}\right)+\frac{\lambda_{2}^{-1}}{2}\left(1+\frac{(1-\gamma)(1-\beta \gamma)}{\gamma\left(1-\lambda_{2}^{-1}\right)}\right)\right) \\
& =\theta_{\pi}-\frac{\kappa \sigma}{\lambda_{i} \beta \gamma} \frac{(1-\gamma)(1-\beta \gamma)}{\left(1-\lambda_{2}\right)\left(1-\lambda_{3}\right)}\left(1-\frac{\lambda_{2}+\lambda_{3}}{2}\right) \\
& \geq \theta_{\pi} .
\end{aligned}
$$


As for rule I, we can rewrite (A.35) as in (A.37), but where the coefficients are given in (A.40), (A.41), and (A.45) - (A.47). Again, we know from Proposition 9 that (2.5) holds, and thus that (A.38) holds. Multiplying (A.38) on both sides by $\left(1-\lambda_{2}\right)^{-1}\left(1-\frac{\lambda_{2}+\lambda_{3}}{2}\right)$, we obtain

$$
\begin{aligned}
\left(1-\lambda_{1}\right)\left(1-\frac{\lambda_{2}+\lambda_{3}}{2}\right) i^{*}= & \left(1-\lambda_{1}\right)\left(1-\frac{\lambda_{2}+\lambda_{3}}{2}\right) \bar{\imath} \\
& +\frac{\kappa \sigma}{\lambda_{i}} \frac{(1-\gamma)(1-\beta \gamma)}{\beta \gamma\left(1-\lambda_{2}\right)\left(1-\lambda_{3}\right)}\left(1-\frac{\lambda_{2}+\lambda_{3}}{2}\right) \bar{\pi}
\end{aligned}
$$

Since (A.40) and (A.48) imply

$$
\begin{aligned}
\left(1-\lambda_{1}\right)\left(1-\frac{\lambda_{2}+\lambda_{3}}{2}\right) & =1-\rho_{1} \\
\frac{\kappa \sigma}{\lambda_{i} \beta \gamma} \frac{(1-\gamma)(1-\beta \gamma)}{\left(1-\lambda_{2}\right)\left(1-\lambda_{3}\right)}\left(1-\frac{\lambda_{2}+\lambda_{3}}{2}\right) & =-\left(\phi_{\pi}-\theta_{\pi}\right),
\end{aligned}
$$

we can rewrite (A.49) as

$$
\left(1-\rho_{1}\right) i^{*}=\left(1-\rho_{1}\right) \bar{\imath}-\left(\phi_{\pi}-\theta_{\pi}\right) \bar{\pi}
$$

Combining this with (A.37) yields (A.26).

Finally, in the limit, as $\gamma=1$, we have

$$
\phi_{\pi}=\frac{\kappa \sigma}{\lambda_{i} \beta} \frac{\lambda_{2}^{-1}+\lambda_{3}^{-1}}{2}=\theta_{\pi} .
$$

\section{A.9 Proposition 11}

Proposition 11. Under the assumptions of Proposition 8, a pair of bounded processes $\left\{\hat{\imath}_{t}, \hat{f}_{t}\right\}$ satisfy

$$
\left(1-\lambda_{1} L\right) \hat{\imath}_{t-1}=-\left(\beta \gamma \lambda_{2} \lambda_{3}\right)^{-1} \mathrm{E}_{t}\left[\left(1-\lambda_{2}^{-1} L^{-1}\right)^{-1}\left(1-\lambda_{3}^{-1} L^{-1}\right)^{-1} \hat{f}_{t}\right]
$$

at all dates $t \geq t_{0}$ if and only if they satisfy

$$
\mathrm{E}_{t}\left[A(L) \hat{\imath}_{t+1}\right]=-\hat{f}_{t}
$$

at all of those same dates. 
Proof: Proposition 8 guarantees that $0<\lambda_{1}<1$, and that $\lambda_{2}, \lambda_{3}$ are either real values, or complex conjugates, that lie outside the unit circle.

First we show that (A.51) implies (A.50). Using (2.9) to substitute for $A(L)$, and expanding the left-hand side of (A.51), we obtain

$$
\beta \gamma \lambda_{2} \lambda_{3} \mathrm{E}_{t}\left[\left(1-\lambda_{1} L\right)\left(\lambda_{2}^{-1} L^{-1}-1\right)\left(\lambda_{3}^{-1} L^{-1}-1\right) L^{2} \hat{\imath}_{t+1}\right]=-\hat{f}_{t},
$$

or

$$
\mathrm{E}_{t}\left[D(L)\left(1-\lambda_{3}^{-1} L^{-1}\right) \hat{\imath}_{t-1}\right]=v_{t}
$$

where

$$
\begin{aligned}
D(L) & =\left(1-\lambda_{1} L\right)\left(1-\lambda_{2}^{-1} L^{-1}\right) \\
v_{t} & \equiv-\left(\beta \gamma \lambda_{2} \lambda_{3}\right)^{-1} \hat{f}_{t} .
\end{aligned}
$$

It follows that

$$
\begin{aligned}
\mathrm{E}_{t}\left[D(L) \hat{\imath}_{t-1}\right] & =\lambda_{3}^{-1} \mathrm{E}_{t}\left[D(L) \hat{\imath}_{t}\right]+v_{t} \\
& =\mathrm{E}_{t}\left[\sum_{j=0}^{\infty} \lambda_{3}^{-j} v_{t+j}\right] \\
& =\mathrm{E}_{t}\left[\left(1-\lambda_{3}^{-1} L^{-1}\right)^{-1} v_{t}\right] .
\end{aligned}
$$

This can furthermore be rewritten as

$$
\mathrm{E}_{t}\left[\left(1-\lambda_{1} L\right)\left(1-\lambda_{2}^{-1} L^{-1}\right) \hat{\imath}_{t-1}\right]=\tilde{v}_{t},
$$

where

$$
\tilde{v}_{t} \equiv-\left(\beta \gamma \lambda_{2} \lambda_{3}\right)^{-1} \mathrm{E}_{t}\left[\left(1-\lambda_{3}^{-1} L^{-1}\right)^{-1} \hat{f}_{t}\right]
$$

It follows that

$$
\begin{aligned}
\mathrm{E}_{t}\left[\left(1-\lambda_{1} L\right) \hat{\imath}_{t-1}\right] & =\lambda_{2}^{-1} \mathrm{E}_{t}\left[\left(1-\lambda_{1} L\right) \hat{\imath}_{t}\right]+\tilde{v}_{t} \\
& =\mathrm{E}_{t}\left[\sum_{j=0}^{\infty} \lambda_{2}^{-j} \tilde{v}_{t+j}\right] \\
& =\mathrm{E}_{t}\left[\left(1-\lambda_{2}^{-1} L^{-1}\right)^{-1} \tilde{v}_{t}\right] \\
& =-\left(\beta \gamma \lambda_{2} \lambda_{3}\right)^{-1} \mathrm{E}_{t}\left[\left(1-\lambda_{2}^{-1} L^{-1}\right)^{-1}\left(1-\lambda_{3}^{-1} L^{-1}\right)^{-1} \hat{f}_{t}\right]
\end{aligned}
$$


which corresponds to (A.50).

We next show that (A.50) implies (A.51). Since (A.50) holds for all $t \geq t_{0}$, it implies that

$$
\left(1-\lambda_{1} L\right) \hat{\imath}_{t+1}=-\left(\beta \gamma \lambda_{2} \lambda_{3}\right)^{-1} \mathrm{E}_{t+2}\left[\left(1-\lambda_{2}^{-1} L^{-1}\right)^{-1}\left(1-\lambda_{3}^{-1} L^{-1}\right)^{-1} \hat{f}_{t+2}\right] .
$$

Multiplying on both sides by $\beta \gamma\left(1-\lambda_{2} L\right)\left(1-\lambda_{3} L\right)$, taking conditional expectations at date $t$, and using (2.9), we obtain

$$
\begin{aligned}
\mathrm{E}_{t}\left[A(L) \hat{\imath}_{t+1}\right] & =-\lambda_{2}^{-1} \lambda_{3}^{-1} \mathrm{E}_{t}\left[\left(1-\lambda_{2} L\right)\left(1-\lambda_{3} L\right)\left(1-\lambda_{2}^{-1} L^{-1}\right)^{-1}\left(1-\lambda_{3}^{-1} L^{-1}\right)^{-1} \hat{f}_{t+2}\right] \\
& =-\mathrm{E}_{t}\left[L^{2} \hat{f}_{t+2}\right] \\
& =-\hat{f}_{t} .
\end{aligned}
$$

which corresponds to (A.51).

\section{A.10 Proposition 12}

Proposition 12. Under the assumptions of Proposition 10, the optimal target criterion

$$
\left(1-\lambda_{1} L\right) \hat{\imath}_{t-1}=-\left(\beta \gamma \lambda_{2} \lambda_{3}\right)^{-1} \mathrm{E}_{t}\left[\left(1-\lambda_{2}^{-1} L^{-1}\right)^{-1}\left(1-\lambda_{3}^{-1} L^{-1}\right)^{-1} \hat{f}_{t}\right]
$$

has a representation

$$
F_{t}(\pi)+\frac{\phi_{x}}{4} F_{t}(x)=\theta_{\pi} \pi_{t-1}+\frac{\theta_{x}}{4} x_{t-1}-\theta_{i}\left(i_{t-1}-i^{*}\right)-\theta_{\Delta} \Delta i_{t-1}
$$

in which

$$
\begin{aligned}
\phi_{x} & =\theta_{x}>0, \\
0 & <\theta_{\pi} \leq 1,
\end{aligned}
$$

and

$$
\theta_{i}, \theta_{\Delta}>0
$$

Furthermore, for fixed values of the other parameters, as $\gamma \rightarrow 0, \theta_{\pi}$ approaches zero and the other parameters approach the non-zero values associated with the target criterion (1.25). Instead, as $\gamma \rightarrow 1, \theta_{\pi}$ approaches 1 . 
Proof: The optimal target criterion (A.52) can be written as

$$
\hat{\imath}_{t-1}-\lambda_{1} \hat{\imath}_{t-2}=-\left(\beta \gamma \lambda_{2} \lambda_{3}\right)^{-1} v_{t}
$$

where

$$
v_{t} \equiv \mathrm{E}_{t}\left[\left(1-\lambda_{2}^{-1} L^{-1}\right)^{-1}\left(1-\lambda_{3}^{-1} L^{-1}\right)^{-1} \hat{f}_{t}\right]
$$

and where $\left|\lambda_{2}\right|,\left|\lambda_{3}\right|>1$. We note that in the case that $\lambda_{2} \neq \lambda_{3}$,

$$
\left(1-\lambda_{2}^{-1} L^{-1}\right)^{-1}\left(1-\lambda_{3}^{-1} L^{-1}\right)^{-1}=c_{2}\left(1-\lambda_{2}^{-1} L^{-1}\right)^{-1}-c_{3}\left(1-\lambda_{3}^{-1} L^{-1}\right)^{-1}
$$

where $c_{2} \equiv \lambda_{3} /\left(\lambda_{3}-\lambda_{2}\right)$ and $c_{3} \equiv \lambda_{2} /\left(\lambda_{3}-\lambda_{2}\right)$. The variable $v_{t}$ can therefore be rewritten as

$$
\begin{aligned}
v_{t} & =\mathrm{E}_{t}\left\{\left[c_{2}\left(1-\lambda_{2}^{-1} L^{-1}\right)^{-1}-c_{3}\left(1-\lambda_{3}^{-1} L^{-1}\right)^{-1}\right] \hat{f}_{t}\right\} \\
& =\mathrm{E}_{t}\left[\sum_{j=0}^{\infty}\left(c_{2} \lambda_{2}^{-j}-c_{3} \lambda_{3}^{-j}\right) \hat{f}_{t+j}\right] .
\end{aligned}
$$

Substituting for $\hat{f}_{t+j}$ as in the proof of Proposition 10, we obtain

$$
\begin{aligned}
v_{t}= & \frac{\kappa \sigma}{\lambda_{i}} \mathrm{E}_{t}\left[\sum_{j=0}^{\infty}\left(c_{2} \lambda_{2}^{-j}-c_{3} \lambda_{3}^{-j}\right)\left(-\gamma \hat{\pi}_{t+j-1}+\left(1+\beta \gamma^{2}\right) \hat{\pi}_{t+j}-\beta \gamma \hat{\pi}_{t+j+1}\right)\right] \\
& +\frac{\lambda_{x} \sigma}{\lambda_{i}} \mathrm{E}_{t}\left[\sum_{j=0}^{\infty}\left(c_{2} \lambda_{2}^{-j}-c_{3} \lambda_{3}^{-j}\right)\left(-\hat{x}_{t+j-1}+(1+\beta \gamma) \hat{x}_{t+j}-\beta \gamma \hat{x}_{t+j+1}\right)\right] \\
= & \frac{\kappa \sigma}{\lambda_{i}} \sum_{j=-1}^{\infty} \tilde{\alpha}_{\pi, j} \mathrm{E}_{t} \hat{\pi}_{t+j}+\frac{\lambda_{x} \sigma}{\lambda_{i}} \sum_{j=-1}^{\infty} \alpha_{x, j} \mathrm{E}_{t} \hat{x}_{t+j},
\end{aligned}
$$

where

$$
\begin{aligned}
\tilde{\alpha}_{\pi,-1} & =-\gamma \\
\tilde{\alpha}_{\pi, 0} & =1+\beta \gamma^{2}-\left(c_{2} \lambda_{2}^{-1}-c_{3} \lambda_{3}^{-1}\right) \gamma \\
\tilde{\alpha}_{\pi, j} & =-\left(c_{2} \lambda_{2}^{-j+1}-c_{3} \lambda_{3}^{-j+1}\right) \beta \gamma+\left(c_{2} \lambda_{2}^{-j}-c_{3} \lambda_{3}^{-j}\right)\left(1+\beta \gamma^{2}\right)-\left(c_{2} \lambda_{2}^{-j-1}-c_{3} \lambda_{3}^{-j-1}\right) \gamma,
\end{aligned}
$$

$\forall j \geq 1$, and

$$
\begin{aligned}
\alpha_{x,-1} & =-1 \\
\alpha_{x, 0} & =1+\beta \gamma-\left(c_{2} \lambda_{2}^{-1}-c_{3} \lambda_{3}^{-1}\right) \\
\alpha_{x, j} & =-\left(c_{2} \lambda_{2}^{-j+1}-c_{3} \lambda_{3}^{-j+1}\right) \beta \gamma+\left(c_{2} \lambda_{2}^{-j}-c_{3} \lambda_{3}^{-j}\right)(1+\beta \gamma)-\left(c_{2} \lambda_{2}^{-j-1}-c_{3} \lambda_{3}^{-j-1}\right),
\end{aligned}
$$


$\forall j \geq 1$. We can furthermore write the variable $v_{t}$ as

$$
v_{t}=\frac{\kappa \sigma}{\lambda_{i}} S_{\pi} \sum_{j=0}^{\infty} \alpha_{\pi, j} \mathrm{E}_{t} \hat{\pi}_{t+j}+\frac{\lambda_{x} \sigma}{\lambda_{i}} \sum_{j=0}^{\infty} \alpha_{x, j} \mathrm{E}_{t} \hat{x}_{t+j}-\frac{\kappa \sigma \gamma}{\lambda_{i}} \hat{\pi}_{t-1}-\frac{\lambda_{x} \sigma}{\lambda_{i}} \hat{x}_{t-1} .
$$

where

$$
\begin{aligned}
S_{\pi} & =\sum_{j=0}^{\infty} \tilde{\alpha}_{\pi, j}=\frac{\lambda_{3}\left(1+\beta \gamma^{2}-\beta \gamma-\lambda_{2}^{-1} \gamma\right)}{\left(\lambda_{3}-\lambda_{2}\right)\left(1-\lambda_{2}^{-1}\right)}-\frac{\lambda_{2}\left(1+\beta \gamma^{2}-\beta \gamma-\lambda_{3}^{-1} \gamma\right)}{\left(\lambda_{3}-\lambda_{2}\right)\left(1-\lambda_{3}^{-1}\right)} \\
& =\gamma+\frac{(1-\gamma)(1-\beta \gamma)}{\left(1-\lambda_{2}^{-1}\right)\left(1-\lambda_{3}^{-1}\right)} \\
& \geq \gamma
\end{aligned}
$$

and

$$
\alpha_{\pi, j}=\frac{\tilde{\alpha}_{\pi, j}}{S_{\pi}}, \quad \forall j \geq 0 .
$$

Note that the coefficients $\alpha_{\pi, j}$ and $\alpha_{x, j}$ satisfy

$$
\sum_{j=0}^{\infty} \alpha_{\pi, j}=\sum_{j=0}^{\infty} \alpha_{x, j}=1
$$

Combining (A.54) and (A.55), we can rewrite the optimal target criterion as

$$
\frac{\kappa \sigma}{\lambda_{i}} S_{\pi} F_{t}(\hat{\pi})+\frac{\lambda_{x} \sigma}{\lambda_{i}} F_{t}(\hat{x})-\frac{\kappa \sigma \gamma}{\lambda_{i}} \hat{\pi}_{t-1}-\frac{\lambda_{x} \sigma}{\lambda_{i}} \hat{x}_{t-1}=-\beta \gamma \lambda_{2} \lambda_{3}\left(\hat{\imath}_{t-1}-\lambda_{1} \hat{\imath}_{t-2}\right) .
$$

Recalling that $\hat{z}_{t} \equiv z_{t}-\bar{z}$ for any variable $z$, we have

$$
\begin{aligned}
\frac{\kappa \sigma}{\lambda_{i}} S_{\pi}\left(F_{t}(\pi)-\bar{\pi}\right)+\frac{\lambda_{x} \sigma}{\lambda_{i}} F_{t}(x)= & \frac{\kappa \sigma \gamma}{\lambda_{i}}\left(\pi_{t-1}-\bar{\pi}\right)+\frac{\lambda_{x} \sigma}{\lambda_{i}} x_{t-1} \\
& -\beta \gamma \lambda_{2} \lambda_{3}\left(\left(1-\lambda_{1}\right)\left(i_{t-1}-\bar{\imath}\right)-\lambda_{1} \Delta i_{t-2}\right),
\end{aligned}
$$

or an equation of the form

$$
F_{t}(\pi)+\frac{\phi_{x}}{4} F_{t}(x)=\theta_{i} \bar{\imath}+\left(1-\theta_{\pi}\right) \bar{\pi}+\theta_{\pi} \pi_{t-1}+\frac{\theta_{x}}{4} x_{t-1}-\theta_{i} i_{t-1}-\theta_{\Delta} \Delta i_{t-1}
$$

where

$$
\begin{aligned}
\phi_{x} & =\theta_{x}=\frac{4 \lambda_{x}}{\kappa S_{\pi}}>0 \\
\theta_{\pi} & =\frac{\gamma}{S_{\pi}}>0 \\
\theta_{i} & =\frac{\lambda_{i} \beta \gamma\left(1-\lambda_{1}\right) \lambda_{2} \lambda_{3}}{\kappa \sigma S_{\pi}}>0 \\
\theta_{\Delta} & =\frac{\lambda_{i} \beta \gamma \lambda_{1} \lambda_{2} \lambda_{3}}{\kappa \sigma S_{\pi}}>0 .
\end{aligned}
$$


We know from Proposition 9 that (2.10) holds, and thus that (2.5) holds. In the steady state, equation $(2.5)$ reduces to

$$
A(L)\left(\bar{\imath}-i^{*}\right)=-\bar{f}
$$

where

$$
\bar{f}=\frac{\kappa \sigma}{\lambda_{i}}(1-\beta \gamma) \bar{q}=\frac{\kappa \sigma}{\lambda_{i}}(1-\beta \gamma)(1-\gamma) \bar{\pi} .
$$

Using (2.9), we have furthermore

$$
\begin{aligned}
\theta_{i} i^{*} & =\theta_{i} \bar{\imath}+\frac{\theta_{i}}{A(1)} \frac{\kappa \sigma}{\lambda_{i}}(1-\beta \gamma)(1-\gamma) \bar{\pi} \\
& =\theta_{i} \bar{\imath}+\frac{\frac{\lambda_{i} \beta \gamma\left(1-\lambda_{1}\right) \lambda_{2} \lambda_{3}}{\kappa \sigma S_{\pi}}}{\beta \gamma\left(1-\lambda_{1}\right)\left(1-\lambda_{2}\right)\left(1-\lambda_{3}\right)} \frac{\kappa \sigma}{\lambda_{i}}(1-\beta \gamma)(1-\gamma) \bar{\pi} \\
& =\theta_{i} \bar{\imath}+\frac{(1-\beta \gamma)(1-\gamma)}{\left(1-\lambda_{2}^{-1}\right)\left(1-\lambda_{3}^{-1}\right)} \frac{1}{S_{\pi}} \bar{\pi} \\
& =\theta_{i} \bar{\imath}+\left(S_{\pi}-\gamma\right) \frac{1}{S_{\pi}} \bar{\pi} \\
& =\theta_{i} \bar{\imath}+\left(1-\theta_{\pi}\right) \bar{\pi}
\end{aligned}
$$

Combining this with (A.57) yields (A.53).

As $\gamma$ approaches 0 , it follows from Lemma 2 below that $\lambda_{3}^{-1} \rightarrow 0, \lambda_{3} \gamma \rightarrow \beta^{-1}, S_{\pi} \rightarrow$ $\left(1-\lambda_{2}^{-1}\right)^{-1}>0$, and thus

$$
\begin{aligned}
\phi_{x} & =\theta_{x} \rightarrow 4\left(1-\lambda_{2}^{-1}\right) \frac{\lambda_{x}}{\kappa}>0 \\
\theta_{\pi} & \rightarrow 0 \\
\theta_{i} & \rightarrow \lambda_{2}\left(1-\lambda_{1}\right)\left(1-\lambda_{2}^{-1}\right) \frac{\lambda_{i}}{\kappa \sigma}>0 \\
\theta_{\Delta} & \rightarrow \lambda_{1} \lambda_{2}\left(1-\lambda_{2}^{-1}\right) \frac{\lambda_{i}}{\kappa \sigma}>0 .
\end{aligned}
$$

Furthermore, $c_{2}=\lambda_{2}^{-1} /\left(\lambda_{2}^{-1}-\lambda_{3}^{-1}\right) \rightarrow 1, c_{3}=\lambda_{3}^{-1} /\left(\lambda_{2}^{-1}-\lambda_{3}^{-1}\right) \rightarrow 0$, and $\tilde{\alpha}_{\pi, j} \rightarrow \lambda_{2}^{-j}$, for all $j \geq 0$, so that

$$
\begin{aligned}
& \alpha_{\pi, j} \rightarrow\left(1-\lambda_{2}^{-1}\right) \lambda_{2}^{-j} \\
& \alpha_{x, j} \rightarrow\left(1-\lambda_{2}^{-1}\right) \lambda_{2}^{-j},
\end{aligned}
$$


for all $j \geq 0$. Instead, as $\gamma=1$, we have $S_{\pi} \rightarrow 1$, and

$$
\theta_{\pi} \rightarrow 1
$$

So far, we have considered the case in which $\lambda_{2} \neq \lambda_{3}$. Now suppose, alternatively, that $\lambda_{2}=\lambda_{3}$. In that case, since

$$
\left(1-\lambda_{2}^{-1} L^{-1}\right)^{-1}\left(1-\lambda_{3}^{-1} L^{-1}\right)^{-1}=\left(1-\lambda_{2}^{-1} L^{-1}\right)^{-2}=\sum_{j=0}^{\infty}(1+j) \lambda_{2}^{-j} L^{-j}
$$

we can write $v_{t}$ as

$$
v_{t}=\mathrm{E}_{t}\left[\sum_{j=0}^{\infty}(1+j) \lambda_{2}^{-j} \hat{f}_{t+j}\right]
$$

Substituting for $\hat{f}_{t+j}$ as in the proof of Proposition 10, we obtain

$$
\begin{aligned}
v_{t}= & \frac{\kappa \sigma}{\lambda_{i}} \mathrm{E}_{t}\left[\sum_{j=0}^{\infty}(1+j) \lambda_{2}^{-j}\left(-\gamma \hat{\pi}_{t+j-1}+\left(1+\beta \gamma^{2}\right) \hat{\pi}_{t+j}-\beta \gamma \hat{\pi}_{t+j+1}\right)\right] \\
& +\frac{\lambda_{x} \sigma}{\lambda_{i}} \mathrm{E}_{t}\left[\sum_{j=0}^{\infty}(1+j) \lambda_{2}^{-j}\left(-\hat{x}_{t+j-1}+(1+\beta \gamma) \hat{x}_{t+j}-\beta \gamma \hat{x}_{t+j+1}\right)\right] \\
= & \frac{\kappa \sigma}{\lambda_{i}} \sum_{j=-1}^{\infty} \tilde{\alpha}_{\pi, j} \mathrm{E}_{t} \hat{\pi}_{t+j}+\frac{\lambda_{x} \sigma}{\lambda_{i}} \sum_{j=-1}^{\infty} \alpha_{x, j} \mathrm{E}_{t} \hat{x}_{t+j},
\end{aligned}
$$

where

$$
\begin{aligned}
\tilde{\alpha}_{\pi,-1} & =-\gamma \\
\tilde{\alpha}_{\pi, 0} & =1+\beta \gamma^{2}-2 \lambda_{2}^{-1} \gamma \\
\tilde{\alpha}_{\pi, j} & =-j \lambda_{2}^{-j+1} \beta \gamma+(1+j) \lambda_{2}^{-j}\left(1+\beta \gamma^{2}\right)-(2+j) \lambda_{2}^{-j-1} \gamma, \quad \forall j \geq 1
\end{aligned}
$$

and

$$
\begin{aligned}
\alpha_{x,-1} & =-1 \\
\alpha_{x, 0} & =1+\beta \gamma-2 \lambda_{2}^{-1} \\
\alpha_{x, j} & =-j \lambda_{2}^{-j+1} \beta \gamma+(1+j) \lambda_{2}^{-j}(1+\beta \gamma)-(2+j) \lambda_{2}^{-j-1}, \quad \forall j \geq 1
\end{aligned}
$$


The variable $v_{t}$ can again be written as in (A.55), where as before $\alpha_{\pi, j}=\frac{\tilde{\alpha}_{\pi, j}}{S_{\pi}}, \forall j \geq 0$, and

$$
\begin{aligned}
S_{\pi}= & \sum_{j=0}^{\infty} \tilde{\alpha}_{\pi, j}=-\left(1 \lambda_{2}^{-0}+2 \lambda_{2}^{-1}+3 \lambda_{2}^{-2}+\ldots\right) \beta \gamma+\left(1 \lambda_{2}^{-0}+2 \lambda_{2}^{-1}+3 \lambda_{2}^{-2}+\ldots\right)\left(1+\beta \gamma^{2}\right) \\
& -\left(2 \lambda_{2}^{-1}+3 \lambda_{2}^{-2}+\ldots\right) \gamma \\
= & \left(1-\lambda_{2}^{-1}\right)^{-2}\left(-\beta \gamma+1+\beta \gamma^{2}-\gamma\right)+\gamma \\
= & \gamma+\frac{(1-\gamma)(1-\beta \gamma)}{\left(1-\lambda_{2}^{-1}\right)^{2}} .
\end{aligned}
$$

Note that $S_{\pi}$ is equal to the value obtained in (A.56), when $\lambda_{2}=\lambda_{3}$. In addition, the coefficients $\alpha_{\pi, j}$ and $\alpha_{x, j}$ satisfy again $\sum_{j=0}^{\infty} \alpha_{\pi, j}=\sum_{j=0}^{\infty} \alpha_{x, j}=1$. It follows that the optimal target criterion can again be expressed as in (A.53) where the coefficients are defined as before.

\section{A.11 Technical Lemmas}

Lemma 1. For any real coefficients $A_{0}, A_{1}, A_{2}, A_{3}$, we have

$$
A_{3} z^{3}+A_{2} z^{2}+A_{1} z+A_{0}=A_{3}\left(B_{0}+B_{1} \zeta+\zeta^{3}\right)
$$

where

$$
\begin{aligned}
\zeta & =z+\frac{A_{2}}{3 A_{3}} \\
B_{0} & =\frac{27 A_{0} A_{3}^{2}+2 A_{2}^{3}-9 A_{3} A_{2} A_{1}}{27 A_{3}^{3}} \\
B_{1} & =\frac{3 A_{3} A_{1}-A_{2}^{2}}{3 A_{3}^{2}} .
\end{aligned}
$$

Proof: $A_{3}\left(B_{0}+B_{1} \zeta+\zeta^{3}\right)$

$$
\begin{aligned}
& =A_{3}\left(\zeta^{3}+\frac{1}{3} \frac{3 A_{3} A_{1}-A_{2}^{2}}{A_{3}^{2}} \zeta+\frac{1}{27} \frac{27 A_{0} A_{3}^{2}+2 A_{2}^{3}-9 A_{3} A_{2} A_{1}}{A_{3}^{3}}\right) \\
& =A_{3}\left(\left(z+\frac{1}{3} \frac{A_{2}}{A_{3}}\right)^{3}+\frac{1}{3} \frac{3 A_{3} A_{1}-A_{2}^{2}}{A_{3}^{2}}\left(z+\frac{1}{3} \frac{A_{2}}{A_{3}}\right)+\frac{1}{27} \frac{27 A_{0} A_{3}^{2}+2 A_{2}^{3}-9 A_{3} A_{2} A_{1}}{A_{3}^{3}}\right) \\
& =A_{3} z^{3}+A_{2} z^{2}+\left(\frac{1}{3} \frac{A_{2}^{2}}{A_{3}}+\frac{1}{3} \frac{3 A_{3} A_{1}-A_{2}^{2}}{A_{3}}\right) z+\frac{1}{27} \frac{A_{2}^{3}}{A_{3}^{2}}+\frac{1}{27} \frac{27 A_{0} A_{3}^{2}+2 A_{2}^{3}-9 A_{3} A_{2} A_{1}}{A_{3}^{2}}+\frac{1}{9} \frac{3 A_{3} A_{1}-A_{2}^{2}}{A_{3}^{2}} A_{2} \\
& =A_{3} z^{3}+A_{2} z^{2}+A_{1} z+A_{0} .
\end{aligned}
$$


LEmma 2. In the limit, as $\gamma$ approaches $0, \lambda_{3} \rightarrow+\infty$, and $\gamma \lambda_{3} \rightarrow \beta^{-1}$.

Proof: In the limit, as $\gamma$ tends to 0 , the polynomial $A(L)$ reduces to

$$
A(L)=\beta^{-1}\left(\beta-(1+\beta+\kappa \sigma) L+L^{2}\right)(-L),
$$

and can again be factorized as

$$
A(L)=\beta^{-1}\left(z_{1}-L\right)\left(z_{2}-L\right)\left(z_{3}-L\right),
$$

where $z_{3}=0$, and $z_{1}, z_{2}$ are the two roots of the second-order polynomial

$$
\tilde{A}(L)=\beta-(1+\beta+\kappa \sigma) L+L^{2} .
$$

Note that since $\tilde{A}(0)=\beta>0$, and $\tilde{A}(1)=-\kappa \sigma<0$, the two roots of $\tilde{A}(L)$ are real and satisfy $0<z_{2}<1<z_{1}$. It follows that

$$
0<\lambda_{1}<1<\lambda_{2}<\lambda_{3}=+\infty
$$

where $\lambda_{j} \equiv z_{j}^{-1}$, for $j=1,2,3$.

Furthermore, as (A.21) holds for all $\gamma$, we have

$$
\lambda_{3} \gamma=\beta^{-1}+\gamma\left(1+\beta^{-1}-\lambda_{1}-\lambda_{2}\right) .
$$

Taking the limit as $\gamma \rightarrow 0$ on both sides and noting that $\lambda_{1}$ and $\lambda_{2}$ are bounded, we obtain

$$
\lim _{\gamma \rightarrow 0} \lambda_{3} \gamma=\beta^{-1}
$$

